\title{
Ruthenium-Catalyzed Tandem Olefin Metathesis-Oxidations
}

\author{
Andrew A. Scholte, My Hyun An, Marc L. Snapper* \\ Department of Chemistry, Merkert Chemistry Center, Boston College, \\ 2609 Beacon Street, Chestnut Hill, MA 02467 \\ marc.snapper@BC.edu
}

SUPPORTING INFORMATION

Table of Contents

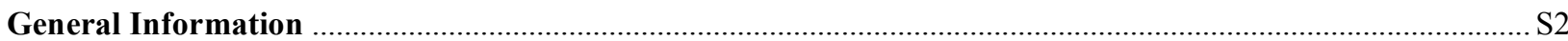

(A) General procedure for tandem ring closing metathesis/ $\alpha$-ketohydroxylation........................................................ S2

(B) General procedure for tandem cross metathesis/ $\alpha$-ketohydroxylation ................................................................ S2

(C) General procedure for tandem ring closing metathesis/dihydroxylation............................................................... S3

(D) General procedure for tandem cross metathesis/dihydroxylation....................................................................... S3

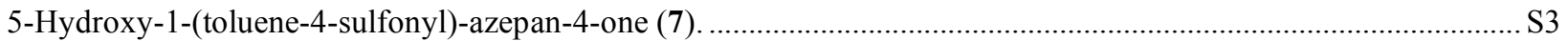

4-Hydroxy-5-oxo-cycloheptane-1,1-dicarboxylic acid diethyl ester (9).................................................................. S4

4-Hydroxy-3-oxo-cyclohexane-1,1-dicarboxylic acid diethyl ester (11a) and 3-hydroxy-4-oxo-cyclohexane-1,1-

dicarboxylic acid diethyl ester (11b).

.. S4

3-Hydroxy-4-oxo-cyclopentane-1,1-dicarboxylic acid diethyl ester (13)................................................................. S5

3-Hydroxy-3-methyl-4-oxo-cyclopentane-1,1-dicarboxylic acid diethyl ester (15).................................................. S5

3-Hydroxy-4-oxo-cyclopentane-1,1,3-tricarboxylic acid diethyl ester methyl ester (17).......................................... S5

3-Hydroxy-4-oxo-3-phenyl-cyclopentane-1,1-dicarboxylic acid diethyl ester (19). ................................................. S6

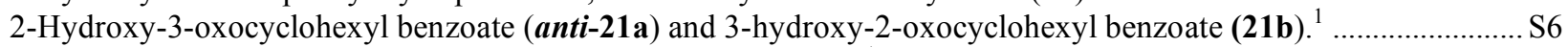

3-Cyclohexyl-2-hydroxy-3-oxo-propionic acid methyl ester (24) ${ }^{1}$......................................................................... S6

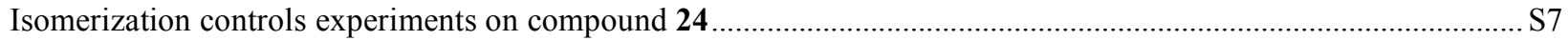

7-Acetoxy-2-hydroxy-3-oxo-heptanoic acid acid methyl ester (26) ........................................................................... S7

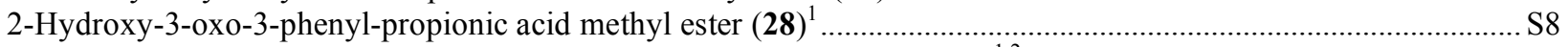

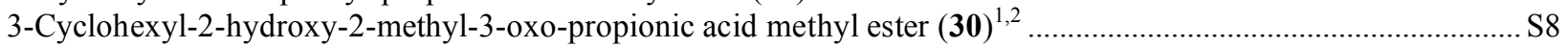

7-Acetoxy-2-hydroxy-2-methyl-3-oxo-heptanoic acid acid methyl ester (31) ........................................................... S8

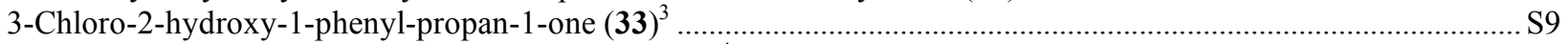

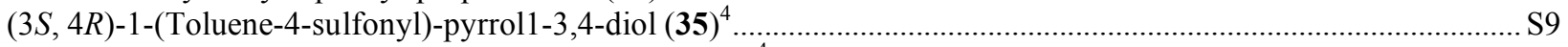

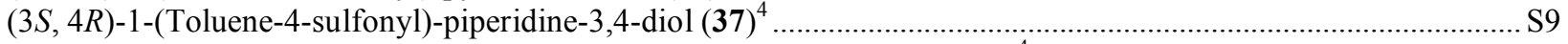

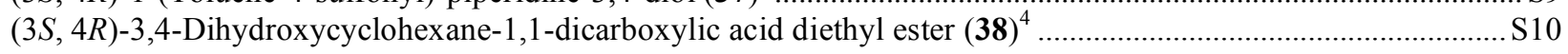

$(4 S, 5 R)-4,5$-Dihydroxycycloheptane-1,1-dicarboxylic acid diethyl ester (39) ...................................................... S10

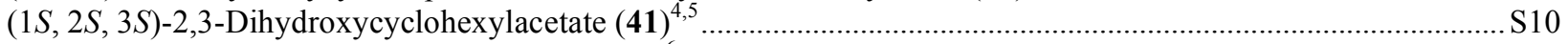

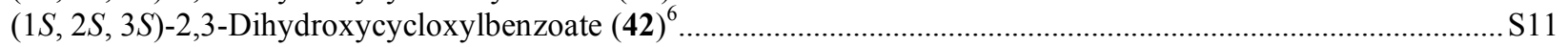

$(3 R, 4 S, 6 R)-6$-Cyclohexyl-tetrahydro-pyran-3,4-diol (44) ..................................................................................... S11

(3R, 4S, 6R)-6-(4-Methoxy-phenyl)-1-(toluene-4-sulfonyl)-piperidine-3,4-diol (46) …………............................... S1

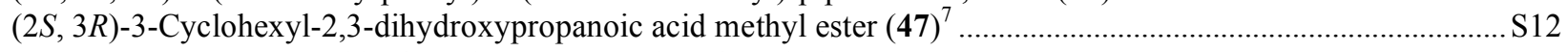

$(2 S, 3 R)$-7-Acetoxy-2,3-dihydroxyheptanoic acid acid methyl ester $(\mathbf{4 8})$.............................................................. S12

$(2 S, 3 R)$-3-Cyclohexyl-2,3-dihydroxy-2-methyl-propanoic acid methyl ester (49) ................................................ S12

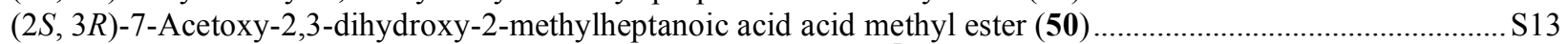

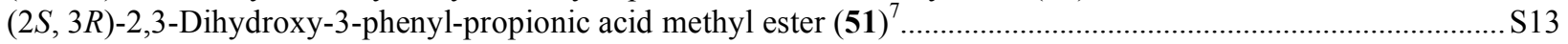

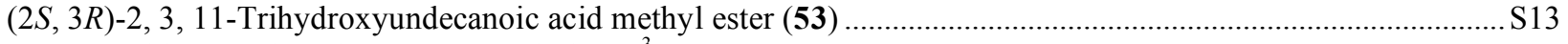

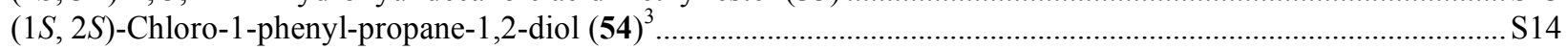

NMR

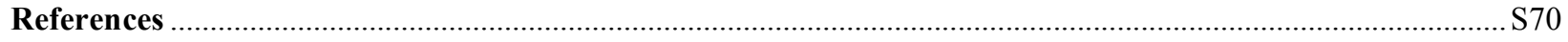




\section{General Information}

All chemicals were purchased from established commercial suppliers. Tetrahydrofuran (THF), diethyl ether $\left(\mathrm{Et}_{2} \mathrm{O}\right)$, and dichloromethane $\left(\mathrm{CH}_{2} \mathrm{Cl}_{2}\right)$ were dried on alumina columns using a solvent dispensing system. ${ }^{*}$ Hexane used in chromatography was distilled prior to use. Acetonitrile $(\mathrm{MeCN})$ was dried over $\mathrm{CaH}_{2}$ and freshly distilled before its use. All reactions were conducted in oven $\left(135^{\circ} \mathrm{C}\right)$ or flame-dried glassware under an inert atmosphere of dry nitrogen. Evaporation refers to removal of solvent under reduced pressure on a rotary evaporator. Analytical TLC was performed on glass plates $(5 \times 1.5 \mathrm{~cm})$ pre-coated $(0.25 \mathrm{~mm})$ with silica gel (EM Science, Kieselgel $60 \mathrm{~F}_{254}$ ). Compounds were visualized by exposure to UV light or by staining with a $1 \% \mathrm{Ce}\left(\mathrm{SO}_{4}\right)_{2} \cdot 4 \mathrm{H}_{2} \mathrm{O} 2.5 \%\left(\mathrm{NH}_{4}\right) \mathrm{Mo}_{7} \mathrm{O}_{24} \cdot 4 \mathrm{H}_{2} \mathrm{O}$ in $10 \% \mathrm{H}_{2} \mathrm{SO}_{4}$ followed by heating on a hot plate. Flash column chromatography was performed with silica gel (60, 230-400 mesh). Vinylcyclohexane (22), styrene (27), methylacrylate (23), methyl methacrylate (29) and octan-1ol (52) were were used following distillation.

Infrared (IR) spectra were recorded on a Nicolet Avatar 360 spectrophotometer or a Mattson Galaxy Series FTIR 5000. Bands are characterized as broad (br), strong (s), medium (m) and weak (w). ${ }^{1}$ H-NMR spectra were recorded on a Varian Unity 300 (300 MHz), Varian Gemini $400(400 \mathrm{MHz})$ or Varian Inova $500(500 \mathrm{MHz})$. Chemical shifts are reported with the solvent as the internal standard $\left(\mathrm{CHCl}_{3} \delta 7.26 \mathrm{ppm}\right)$. Data are reported as follows: chemical shift, integration, multiplicity $(\mathrm{s}=$ singlet, $\mathrm{d}=$ doublet, $\mathrm{t}=$ triplet, $\mathrm{q}=$ quartet, $\mathrm{br}=$ broad, $\mathrm{m}=$ multiplet), and coupling constants (Hz). ${ }^{13} \mathbf{C}-\mathbf{N M R}$ spectra were recorded on a Varian Gemini $400(100 \mathrm{MHz})$ with complete proton decoupling. Chemical shifts are reported in ppm with the solvent as an internal reference $\left(\mathrm{CDCl}_{3} \delta 77.2 \mathrm{ppm}\right)$. A Hewlett Packard HP 6890 Series II Gas Chromatograph was used for all reactions monitored by GC utilizing a Zebron ZB-1 $100 \%$ methyl polysiloxane column. High-resolution mass spectral analyses (HRMS) were performed on a Micromass LCT electrospray mass spectrometer with a TOF analyzer or by the Mass Spectrometry Laboratory at the University of Illinois at Urbana-Champaign.

\section{(A) General procedure for tandem ring-closing metathesis/ $\alpha$-ketohydroxylation}

To a stirring solution of the diene (1 equiv) in EtOAc $(1.2 \mathrm{~mL})$ was added Grubbs' II catalyst 2 (0.05 equiv) and the resulting reaction mixture was stirred at room temperature for $90 \mathrm{~min}$., or the reaction was judged to be complete by TLC or GC/MS analysis. The suspension was diluted with $\mathrm{MeCN}(1.2 \mathrm{~mL})$ and $\mathrm{H}_{2} \mathrm{O}(0.2 \mathrm{~mL})$ and cooled to $0^{\circ} \mathrm{C}$. To the solution was added $\mathrm{NaHCO}_{3}$ (2.5 equiv) and Oxone ${ }^{\circledR}$ (5.0 equiv). After stirring, until the oxidation was judged complete $(\leq 1$ h), the resulting mixture was diluted with EtOAc $(2.0 \mathrm{~mL})$ and filtered. The filtrate was washed with a saturated solution of $\mathrm{Na}_{2} \mathrm{SO}_{3}(3 \mathrm{~mL})$ and dried over $\mathrm{Na}_{2} \mathrm{SO}_{4}$. Filtration and concentration in vacuo gave an amber oil. Purification by flash chromatography afforded the acyloin.

\section{(B) General procedure for tandem cross-metathesis/ $\alpha$-ketohydroxylation}

To a stirring solution of the starting olefin (1 equiv) in $\mathrm{CH}_{2} \mathrm{Cl}_{2}(0.15-0.25 \mathrm{M})$ was added cross-metathesis partner ( 2 equiv) and Grubbs' catalyst 2 ( 0.05 equiv) and the resulting reaction mixture was stirred at reflux until analysis by TLC or GC indicated that the starting olefin was consumed. The suspension was allowed to cool to room temperature and concentrated in vacuo to remove solvent and excess cross-metathesis partner. Residue was dissolved in $\mathrm{MeCN}(3 \mathrm{~mL})$, EtOAc $(3 \mathrm{~mL})$ and $\mathrm{H}_{2} \mathrm{O}(0.5 \mathrm{~mL})$ and cooled to $0{ }^{\circ} \mathrm{C}$. To the solution was added $\mathrm{NaHCO}_{3}(2.5$

\footnotetext{
* Pangborn, A.B.; Giardello, M.A.; Grubbs, R.H.; Rosen, R.K.; Timmers, F.J. Organometallics 1996, $15,1518$.
} 
equiv) and Oxone ${ }^{\circledR}$ (5.0 equiv). After stirring at $0{ }^{\circ} \mathrm{C}$ until the oxidation was complete the mixture was diluted with EtOAc $(2.0 \mathrm{~mL})$ and filtered. The filtrate was washed with a saturated solution of $\mathrm{Na}_{2} \mathrm{SO}_{3}(3 \mathrm{~mL})$ and dried over $\mathrm{Na}_{2} \mathrm{SO}_{4}$. Filtration and concentration in vacuo gave an amber oil. Purification by flash chromatography afforded the acyloin.

\section{(C) General procedure for tandem ring-closing metathesis/dihydroxylation}

To a stirring solution of the diene (1 equiv) in EtOAc $(3 \mathrm{~mL})$ was added Grubbs' catalyst 2 ( 0.05 equiv) and the resulting reaction mixture was stirred at room temperature for $90 \mathrm{~min}$. or the reaction was judged to be complete by TLC or GC/MS analysis. In another flask, a suspension of $\mathrm{NaIO}_{4}\left(1.5\right.$ equiv) in $\mathrm{H}_{2} \mathrm{O}(0.3 \mathrm{~mL})$ was treated with $\mathrm{CeCl}_{3} \cdot 7 \mathrm{H}_{2} \mathrm{O}(0.1$ equiv) and heated gently until suspension was bright yellow, at this point the suspension was diluted with $\mathrm{MeCN}(3 \mathrm{~mL})$ and cooled to $0{ }^{\circ} \mathrm{C}$. To the yellow suspension was then added metathesis solution and stirred at $0{ }^{\circ} \mathrm{C}$ until oxidation was complete (10-20 min.). The reaction was quenched with the addition of solid $\mathrm{Na}_{2} \mathrm{SO}_{3}$ and $\mathrm{Na}_{2} \mathrm{SO}_{4}$ and after stirring for 10 min., the solution was filtered through Celite ( $1 \mathrm{~cm}$ plug). The filtrate was concentrated in vacuo and the residue was purified by flash chromatography to give the diol.

\section{(D) General procedure for tandem cross-metathesis/dihydroxylation}

To a stirring solution of starting olefin (1 equiv) in $\mathrm{CH}_{2} \mathrm{Cl}_{2}(0.15-0.25 \mathrm{M})$ was added the cross-metathesis partner ( 2 equiv) and Grubbs' catalyst 2 ( 0.05 equiv) and the resulting reaction mixture was stirred at reflux until analysis by TLC or GC/MS indicated that the starting olefin was consumed. The suspension was cooled to room temperature and concentrated in vacuo to remove solvent and excess cross-metathesis partner. In another flask, a suspension of $\mathrm{NaIO}_{4}(1.5$ equiv) in $\mathrm{H}_{2} \mathrm{O}(0.3 \mathrm{~mL})$ was treated with $\mathrm{CeCl}_{3} \cdot 7 \mathrm{H}_{2} \mathrm{O}$ (0.1 equiv) and heated gently until the reaction was bright yellow, at this point the suspension was diluted with $\mathrm{MeCN}(3 \mathrm{~mL})$ and cooled to $0{ }^{\circ} \mathrm{C}$. To this yellow suspension was then added the metathesis solution (in $3 \mathrm{~mL}$ of EtOAc) and stirred at $0{ }^{\circ} \mathrm{C}$ until the oxidation was complete $(10-20 \mathrm{~min}$.). The reaction was quenched with the addition of solid $\mathrm{Na}_{2} \mathrm{SO}_{3}$ and $\mathrm{Na}_{2} \mathrm{SO}_{4}$ and after stirring for $10 \mathrm{~min}$., the solution was filtered through Celite $(1 \mathrm{~cm}$ plug). The filtrate was concentrated in vacuo and the residue was purified by flash chromatography to give the diol.

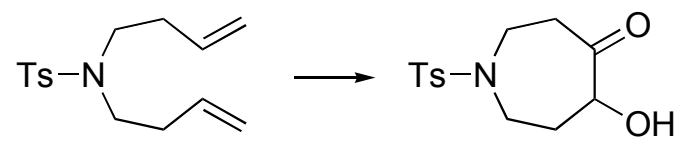

\section{5-Hydroxy-1-(toluene-4-sulfonyl)-azepan-4-one (7).}

General procedure A was followed. $N, N$-Di-but-3-enyl-4-methyl-benzenesulfonamide (6) (20 $\mathrm{mg}, 0.072 \mathrm{mmol})$, Grubbs' catalyst $2(3.0 \mathrm{mg}, 0.0036 \mathrm{mmol}), \mathrm{NaHCO}_{3}(15.0 \mathrm{mg}, 0.18 \mathrm{mmol})$ and Oxone ${ }^{\circledR}(220 \mathrm{mg}, 0.36 \mathrm{mmol})$ were used in this transformation. Purification of the product by flash chromatography $\left(\mathrm{SiO}_{2}\right.$, hexanes:EtOAc, $\left.2: 1\right)$ provided compound 7 as a white solid (12.3 mg, 61\%). mp 113-114 ${ }^{\circ} \mathrm{C}$; IR (thin film, NaCl) 3471, 2924, 1711, 1333, 1158, 1092, 908, $883,868,713,697 \mathrm{~cm}^{-1} ;{ }^{1} \mathbf{H}-\mathbf{N M R}\left(400 \mathrm{MHz}, \mathrm{CDCl}_{3}\right) \delta 7.66(2 \mathrm{H}, \mathrm{d}, J=8.4 \mathrm{~Hz}), 7.32(2 \mathrm{H}, \mathrm{d}, J$ $=8.4 \mathrm{~Hz}), 4.31(1 \mathrm{H}, \mathrm{dt}, J=11.2,3.6 \mathrm{~Hz}), 3.86-3.81(2 \mathrm{H}, \mathrm{m}), 3.77(1 \mathrm{H}, \mathrm{d}, J=4.4 \mathrm{~Hz}), 3.06-2.95$ $(2 \mathrm{H}, \mathrm{m}), 2.89(1 \mathrm{H}, \mathrm{ddd}, J=18.0,10.8,3.6 \mathrm{~Hz}), 2.71(1 \mathrm{H}, \mathrm{ddd}, J=18.0,5.2,2.4 \mathrm{~Hz}), 2.43(3 \mathrm{H}$, s), 2.16-2.10 (1H, m), 1.87-1.77 (1H, m). ${ }^{13}$ C-NMR $\left(100 \mathrm{MHz}, \mathrm{CDCl}_{3}\right) \delta$ 209.6, 143.7, 129.8, 
126.9, 94.3, 76.1, 47.5, 43.6, 41.3, 34.3, 21.6; HRMS (EI) Calc'd for [M+] $\mathrm{C}_{13} \mathrm{H}_{17} \mathrm{NO}_{4} \mathrm{~S}$ 283.0878, found 283.0889.

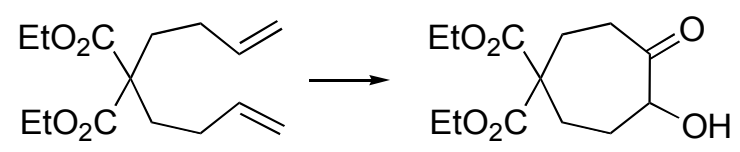

4-Hydroxy-5-oxo-cycloheptane-1,1-dicarboxylic acid diethyl ester (9).

General procedure A was followed. 2,2-Di-but-3-enyl-malonic acid diethyl ester (8) $(30 \mathrm{mg}$, $0.11 \mathrm{mmol})$, Grubbs' catalyst $2(4.8 \mathrm{mg}, 0.056 \mathrm{mmol}), \mathrm{NaHCO}_{3}(24 \mathrm{mg}, 0.28 \mathrm{mmol})$ and Oxone $^{\circledR}$ (344 mg, $0.56 \mathrm{mmol}$ ) were used in this transformation. Purification of the product by flash chromatography $\left(\mathrm{SiO}_{2}\right.$, hexanes:EtOAc, 4:1) provided compound $\mathbf{9}$ as a colorless oil (18 $\mathrm{mg}, 60 \%$ ). IR (thin film, NaCl) 3473, 2981, 1729, 1452, 1297, 1240, 1182, 1095, 1065, 1041 $\mathrm{cm}^{-1}$; ${ }^{1} \mathrm{H}$-NMR $\left(400 \mathrm{MHz}, \mathrm{CDCl}_{3}\right) \delta 4.27(1 \mathrm{H}, \mathrm{dd}, J=8.8,4.0 \mathrm{~Hz}), 4.24-4.12(4 \mathrm{H}, \mathrm{m}), 3.76(1 \mathrm{H}$, bs), $2.77(1 \mathrm{H}$, ddd, $J=16.8,8.4,2.4 \mathrm{~Hz}) 2.51(1 \mathrm{H}, \mathrm{ddd}, J=17.2,11.2,2.8 \mathrm{~Hz}), 2.37-2.30(2 \mathrm{H}$, m), $2.20(1 \mathrm{H}$, dddd, $J=15.2,8.4,2.8,0.8 \mathrm{~Hz}), 2.09-2.01(2 \mathrm{H}, \mathrm{m}), 1.94-1.84(1 \mathrm{H}, \mathrm{m}), 1.27-1.21$ $(6 \mathrm{H}, \mathrm{m}) .{ }^{13} \mathrm{C}-\mathrm{NMR}\left(100 \mathrm{MHz}, \mathrm{CDCl}_{3}\right) \delta$ 211.3, 171.2, 170.1, 76.4, 61.7, 61.6, 57.0, 35.4, 30.7, 28.9, 28.2, 14.1; HRMS (EI) Calc'd for [M+] $\mathrm{C}_{13} \mathrm{H}_{20} \mathrm{O}_{6} 272.1260$, found 272.1259.<smiles>C=CCCC(CC=C)(CC=C)COCC</smiles>

\section{4-Hydroxy-3-oxo-cyclohexane-1,1-dicarboxylic acid diethyl ester (11a) and 3-Hydroxy-4- oxo-cyclohexane-1,1-dicarboxylic acid diethyl ester (11b).}

General procedure A was followed. 2-Allyl-2-but-3-enyl-malonic acid diethyl ester (10) (40 $\mathrm{mg}, 0.16 \mathrm{mmol})$, Grubbs' catalyst $2(6.7 \mathrm{mg}, 0.0079 \mathrm{mmol}), \mathrm{NaHCO}_{3}(33 \mathrm{mg}, 0.39 \mathrm{mmol})$ and Oxone $^{\circledR}$ (484 mg, $0.79 \mathrm{mmol}$ ) were used in this transformation. Purification of the product by flash chromatography $\left(\mathrm{SiO}_{2}\right.$, hexanes:EtOAc, 2:1) provided two regioisomers, compounds 11a (12.5 $\mathrm{mg}, 31 \%$ ) and 11b (8 mg, 20\%) as colorless oils. Data for 11a: IR (thin film, NaCl) 3487, 2982, 1731, 1448, 1368, 1299, 1252, 1086, 1024, $870 \mathrm{~cm}^{-1}$; ${ }^{1} \mathbf{H}-\mathbf{N M R}\left(400 \mathrm{MHz}, \mathrm{CDCl}_{3}\right) \delta$ d.25$4.16(4 \mathrm{H}, \mathrm{m}), 4.09(1 \mathrm{H}, \mathrm{ddd}, J=12.0,6.8,1.2 \mathrm{~Hz}), 3.58(1 \mathrm{H}, \mathrm{bs}), 3.18(1 \mathrm{H}, \mathrm{dd}, J=14.8,3.2$ $\mathrm{Hz}), 2.60(1 \mathrm{H}, \mathrm{dd}, J=14.8,1.6 \mathrm{~Hz}), 2.49(1 \mathrm{H}, \mathrm{ddd}, J=14.4,6.4,3.2 \mathrm{~Hz}), 2.44-2.37$ (1H, ddd, $J$ $=14.4,6.8,3.2 \mathrm{~Hz}), 2.23(1 \mathrm{H}, \mathrm{dt}, J=14.4,3.6 \mathrm{~Hz}), 1.64-1.53(1 \mathrm{H}, \mathrm{m}), 1.26(3 \mathrm{H}, \mathrm{t}, J=6.8 \mathrm{~Hz})$, $1.24(3 \mathrm{H}, \mathrm{t}, J=6.8 \mathrm{~Hz}) ;{ }^{13} \mathbf{C}-\mathrm{NMR}\left(100 \mathrm{MHz}, \mathrm{CDCl}_{3}\right) \delta 206.4,169.5,74.2,62.2,57.7,43.3$, 31.0, 28.8, 14.1; HRMS (ESI) Calc'd for $[\mathrm{M}+\mathrm{H}]^{+} \mathrm{C}_{12} \mathrm{H}_{19} \mathrm{O}_{6}$ 259.1182, found 259.1176.

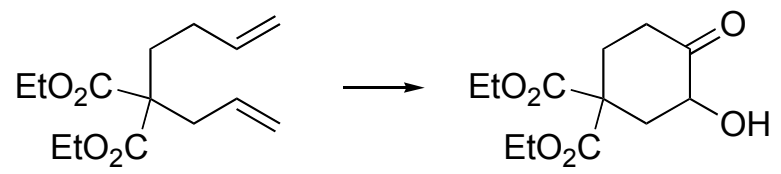

Data for 11b: IR (thin film, NaCl). 3481, 2982, 1731, 1446, 1368, 1297, 1241, 1212, 1181, 1099, 1065, 1024, $859 \mathrm{~cm}^{-1} ;{ }^{1} \mathbf{H}-\mathbf{N M R}\left(400 \mathrm{MHz}, \mathrm{CDCl}_{3}\right) \delta$ 4.33-4.22 $(2 \mathrm{H}, \mathrm{m}), 4.21-4.16(3 \mathrm{H}$, $\mathrm{m}), 3.55(1 \mathrm{H}, \mathrm{d}, J=3.2 \mathrm{~Hz}), 3.03(1 \mathrm{H}, \mathrm{ddd}, J=13.2,6.4,3.2 \mathrm{~Hz}), 2.73-2.52(3 \mathrm{H}, \mathrm{m}), 2.08(1 \mathrm{H}$, dd, $J=13.6,8.4 \mathrm{~Hz}), 1.94(1 \mathrm{H}, \mathrm{dd}, J=13.2,12.4 \mathrm{~Hz}), 1.31(3 \mathrm{H}, \mathrm{t}, J=3.2 \mathrm{~Hz}), 1.25(3 \mathrm{H}, \mathrm{t}, J=$ $6.8 \mathrm{~Hz}) ;{ }^{13} \mathbf{C}-\mathbf{N M R}\left(100 \mathrm{MHz}, \mathrm{CDCl}_{3}\right) \delta 209.2,169.7,71.6,62.1,40.1,35.7,31.9,14.2,14.1$; HRMS (ESI) Calc'd for $[\mathrm{M}+\mathrm{H}]^{+} \mathrm{C}_{12} \mathrm{H}_{19} \mathrm{O}_{6} 259.1182$, found 259.1176 . 


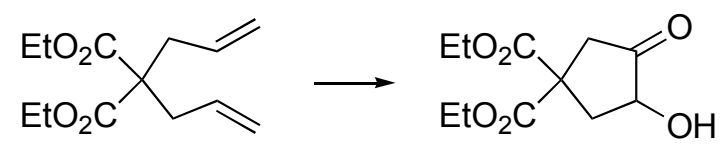

\section{3-Hydroxy-4-oxo-cyclopentane-1,1-dicarboxylic acid diethyl ester (13).}

General procedure A was followed. 2,2-Diallyl-malonic acid diethyl ester (12) (20 mg, 0.083 mmol), Grubbs' catalyst 2 (3.5 mg, $0.0042 \mathrm{mmol}), \mathrm{NaHCO}_{3}(17.5 \mathrm{mg}, 0.21 \mathrm{mmol})$ and Oxone ${ }^{\circledR}$ (256 $\mathrm{mg}, 0.42 \mathrm{mmol}$ ) were used in this transformation. Purification of the product by flash chromatography $\left(\mathrm{SiO}_{2}\right.$, hexanes:EtOAc, 2:1) provided compound $\mathbf{1 3}$ as a colorless oil $(9.3 \mathrm{mg}$, 46\%). IR (thin film, NaCl) 3480, 2984, 1755, 1731, 1393, 1299, 1268, 1249, 1179, 1156, 1097, $859 \mathrm{~cm}^{-1}$; ${ }^{1}$ H-NMR $\left(400 \mathrm{MHz}, \mathrm{CDCl}_{3}\right) \delta 4.30-4.21(5 \mathrm{H}, \mathrm{m}), 3.05-2.99(2 \mathrm{H}, \mathrm{m}), 2.85(1 \mathrm{H}, \mathrm{d}, J=$ $20.0 \mathrm{~Hz}), 2.85(1 \mathrm{H}, \mathrm{bs}), 2.16(1 \mathrm{H}, \mathrm{dd}, J=13.6,11.6 \mathrm{~Hz}), 1.27(3 \mathrm{H}, \mathrm{t}, J=7.2 \mathrm{~Hz}), 1.27(3 \mathrm{H}, \mathrm{t}, J$ $=7.2 \mathrm{~Hz}) .{ }^{13} \mathrm{C}$-NMR $\left(100 \mathrm{MHz}, \mathrm{CDCl}_{3}\right) \delta 212.7,170.6,169.9,73.8,62.5,62.4,52.3,41.5,37.2$, 14.1; HRMS (EI) Calc'd for [M+] $\mathrm{C}_{11} \mathrm{H}_{16} \mathrm{O}_{6} 244.0947$, found 244.0944.

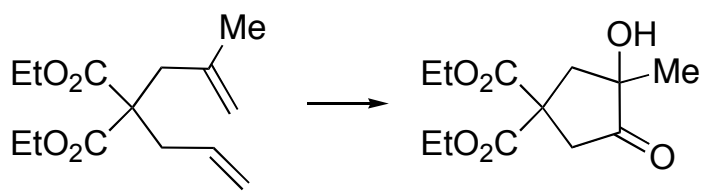

\section{3-Hydroxy-3-methyl-4-oxo-cyclopentane-1,1-dicarboxylic acid diethyl ester (15).}

General procedure A was followed. 2-Allyl-2-(2-methyl-allyl)-malonic acid diethyl ester (14) (20 mg, $0.079 \mathrm{mmol})$, Grubbs' catalyst 2 (6.7 mg, $0.0079 \mathrm{mmol}), \mathrm{NaHCO}_{3}(16.5 \mathrm{mg}, 0.20$ $\mathrm{mmol})$ and Oxone ${ }^{\circledR}(242 \mathrm{mg}, 0.39 \mathrm{mmol})$ were used in this transformation. Purification of the product by flash chromatography $\left(\mathrm{SiO}_{2}\right.$, hexanes:EtOAc, 3:1) provided compound $\mathbf{1 5}$ as a pale yellow oil (10.4 mg, 51\%). IR (thin film, NaCl). 3481, 2983, 1748, 1732, 1392, 1369, 1298, 1252, 1187, 1130, 1096, 1063, $864 \mathrm{~cm}^{-1}$; ${ }^{1} \mathbf{H}-\mathbf{N M R}\left(400 \mathrm{MHz}, \mathrm{CDCl}_{3}\right) \delta 4.23(4 \mathrm{H}, \mathrm{q}, J=7.2$ $\mathrm{Hz}), 3.11(1 \mathrm{H}, \mathrm{dd}, J=19.6,1.6 \mathrm{~Hz}), 2.92(1 \mathrm{H}, \mathrm{dd}, J=19.2,2.0 \mathrm{~Hz}), 2.74(1 \mathrm{H}, \mathrm{dd}, J=14.8,1.6$ $\mathrm{Hz}), 2.68(1 \mathrm{H}, \mathrm{bs}), 2.50(1 \mathrm{H}, \mathrm{d}, J=14.8 \mathrm{~Hz}), 1.29(3 \mathrm{H}, \mathrm{s}), 1.27(3 \mathrm{H}, \mathrm{t}, J=7.2 \mathrm{~Hz}), 1.26(3 \mathrm{H}, \mathrm{t}, J$ $=7.2 \mathrm{~Hz}) ;{ }^{13} \mathbf{C}$-NMR $\left(100 \mathrm{MHz}, \mathrm{CDCl}_{3}\right) \delta 213.4,171.1,170.5,75.9,62.5,62.3,53.5,43.9,42.5$, 23.1, 14.1, 14.1; HRMS (ESI) Calc'd for [M+H] ${ }^{+} \mathrm{C}_{12} \mathrm{H}_{19} \mathrm{O}_{6}$ 259.1182, found 259.1176.

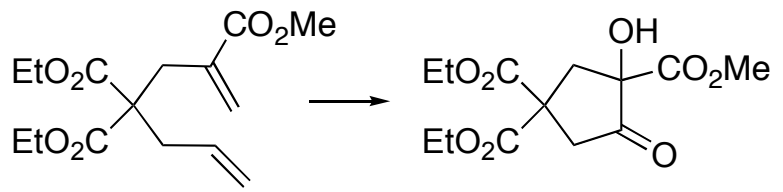

3-Hydroxy-4-oxo-cyclopentane-1,1,3-tricarboxylic acid diethyl ester methyl ester (17).

General procedure A was followed. 2-Allyl-2-ethoxycarbonyl-4-methylene-pentanedioic acid 1-ethyl ester 5-methyl ester (16) (30 mg, $0.10 \mathrm{mmol})$, Grubbs' catalyst $2(8.5 \mathrm{mg}, 0.10 \mathrm{mmol})$, $\mathrm{NaHCO}_{3}(21.1 \mathrm{mg}, 0.25 \mathrm{mmol})$ and Oxone ${ }^{\circledR}(309 \mathrm{mg}, 0.50 \mathrm{mmol})$ were used in this transformation. Purification of the product by flash chromatography $\left(\mathrm{SiO}_{2}\right.$, hexanes:EtOAc, 1:1) provided compound 17 as a colorless oil (16 mg, 53\%). IR (thin film, $\mathrm{NaCl}) 3468,2986,1767$, 1736, 1443, 1369, 1266, 1187, 1008, $860 \mathrm{~cm}^{-1}$; ${ }^{1} \mathbf{H}-\mathbf{N M R}\left(400 \mathrm{MHz}, \mathrm{CDCl}_{3}\right) \delta$ 4.27-4.19 (4H, m), $3.77(4 \mathrm{H}, \mathrm{s}), 3.21(1 \mathrm{H}, \mathrm{dd}, J=18.8,2.0 \mathrm{~Hz}), 3.14(1 \mathrm{H}, \mathrm{d}, J=14.8 \mathrm{~Hz}), 2.94(1 \mathrm{H}, \mathrm{d}, J=19.2$ $\mathrm{Hz}), 2.77(1 \mathrm{H}, \mathrm{dd}, J=14.8,2.0 \mathrm{~Hz}), 1.26(3 \mathrm{H}, \mathrm{t}, J=7.2 \mathrm{~Hz}), 1.25(3 \mathrm{H}, \mathrm{t}, J=7.2 \mathrm{~Hz}) ;{ }^{13} \mathbf{C}-\mathbf{N M R}$ $\left(100 \mathrm{MHz}, \mathrm{CDCl}_{3}\right) \delta$ 207.4, 170.5, 169.9, 169.6, 79.6, 62.5, 62.4, 54.1, 53.4, 43.0, 41.1, 14.1, 14.0; HRMS (ESI) Calc'd for $[\mathrm{M}+\mathrm{H}]^{+} \mathrm{C}_{13} \mathrm{H}_{19} \mathrm{O}_{8} 303.1080$, found 303.1181 . 


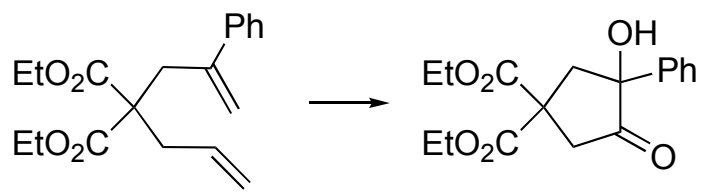

\section{3-Hydroxy-4-oxo-3-phenyl-cyclopentane-1,1-dicarboxylic acid diethyl ester (19).}

General procedure A was followed. Allyl-2-(2-phenyl-allyl)-malonic acid diethyl ester (18) (45 mg, $0.14 \mathrm{mmol}$ ), Grubbs' catalyst 2 (12.1 mg, $0.014 \mathrm{mmol}$ ), $\mathrm{NaHCO}_{3}$ (30 mg, $0.36 \mathrm{mmol}$ ) and Oxone ${ }^{\circledR}$ (437 mg, $0.72 \mathrm{mmol}$ ) were used in this transformation. Purification of the product by flash chromatography $\left(\mathrm{SiO}_{2}\right.$, hexanes:EtOAc, 2:1) provided compound 19 as a pale yellow oil (19.1 mg, 42\%). IR (thin film, NaCl) 3461, 2988, 1748, 1732, 1448, 1368, 1298, 1253, 1187 , 1062, 871, 764, $701 \mathrm{~cm}^{-1}$; ${ }^{1}$ H-NMR (400 MHz, $\left.\mathrm{CDCl}_{3}\right) \delta$ 7.37-7.28 (5H, m), 4.30-4.24 (2H, m), 4.13-4.05 (2H, m), $3.27(1 \mathrm{H}, \mathrm{dd}, J=19.2,2.0 \mathrm{~Hz}), 3.21(1 \mathrm{H}, \mathrm{bs}), 3.07(1 \mathrm{H}, \mathrm{d}, J=19.2 \mathrm{~Hz}), 3.02$ $(1 \mathrm{H}, \mathrm{dd}, J=14.8,1.6 \mathrm{~Hz}), 2.94(1 \mathrm{H}, \mathrm{d}, J=14.8 \mathrm{~Hz}), 1.27(3 \mathrm{H}, \mathrm{t}, J=7.2 \mathrm{~Hz}), 1.19(3 \mathrm{H}, \mathrm{t}, J=7.2$ $\mathrm{Hz}) ;{ }^{13}$ C-NMR $\left(100 \mathrm{MHz}, \mathrm{CDCl}_{3}\right) \delta 212.2,170.9,170.0,139.7,128.4,128.1,125.7,80.1,62.5$, 62.3, 53.7, 45.6, 43.9, 14.1, 14.0; HRMS (ESI) Calc'd for $[\mathrm{M}+\mathrm{Na}]^{+} \mathrm{C}_{17} \mathrm{H}_{20} \mathrm{O}_{6} \mathrm{Na} 343.1158$, found 343.1148 .

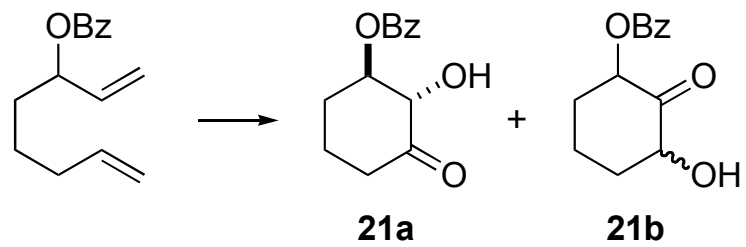

\section{2-Hydroxy-3-oxocyclohexyl benzoate (anti-21a) and 3-Hydroxy-2-oxocyclohexyl benzoate (21b). ${ }^{1}$}

General procedure A was followed. Benzoic acid 1-vinyl-hex-5-enyl ester (20) (20 mg, $0.0 .087 \mathrm{mmol})$, Grubbs' catalyst $2(3.7 \mathrm{mg}, 0.0043 \mathrm{mmol}), \mathrm{NaHCO}_{3}(18 \mathrm{mg}, 0.22 \mathrm{mmol})$ and Oxone $^{\circledR}(267 \mathrm{mg}, 0.43 \mathrm{mmol})$ were used in this transformation. Purification of the product by flash chromatography ( $\mathrm{SiO}_{2}$, hexanes:EtOAc, 2:1) provided compounds 21a and 21b as a 2:1 mixture of regioisomers (12 mg, 60\%). Data for 21a: IR $\left(\mathrm{CH}_{2} \mathrm{Cl}_{2}\right.$ cast) 3401, 2923, 3002, 1719, 1450, $1274 \mathrm{~cm}^{-1} ;{ }^{1} \mathbf{H}-\mathbf{N M R}\left(400 \mathrm{MHz}, \mathrm{CDCl}_{3}\right) \delta 8.07(2 \mathrm{H}, \mathrm{m}), 7.55(1 \mathrm{H}, \mathrm{m}), 7.43$ (2H, m), 4.99 $(1 \mathrm{H}, \mathrm{ddd}, J=11.6,10.0,4.8 \mathrm{~Hz}),(1 \mathrm{H}, \mathrm{m}), 4.34(1 \mathrm{H}, \mathrm{dd}, J=10.0,2.4 \mathrm{~Hz}), 3.69(1 \mathrm{H}, \mathrm{d}, J=4.0$ Hz), $2.63(1 \mathrm{H}$, dqn, $J=14.0,2.4 \mathrm{~Hz}), 2.43-2.37(2 \mathrm{H}, \mathrm{m}), 2.09(1 \mathrm{H}, \mathrm{m}), 1.88(1 \mathrm{H}, \mathrm{ddt}, J=13.2$, 11.2, 4.0 Hz), $1.63(2 \mathrm{H}, \mathrm{m}) ;{ }^{13} \mathrm{C}-\mathrm{NMR}\left(\mathrm{CDCl}_{3}, 100 \mathrm{MHz}\right) \delta 207.0,165.8,133.2,130.0,129.8$, 128.4, 78.9 (2C), 38.8, 29.5, 21.0; HRMS (ESI) Calc'd for $[\mathrm{M}+\mathrm{Na}]^{+} \mathrm{C}_{13} \mathrm{H}_{14} \mathrm{O}_{4} \mathrm{Na} 257.0790$, found 257.0777 .<smiles>C=CC1CCCCC1</smiles>

\section{3-Cyclohexyl-2-hydroxy-3-oxo-propionic acid methyl ester (24). ${ }^{2}$}

General procedure B was followed. Vinylcyclohexane (22) (52 mg, $0.47 \mathrm{mmol}$ ), methylacrylate (23) $(86 \mu \mathrm{L}, 0.94 \mathrm{mmol})$, Grubbs' catalyst 2 (20 mg, $0.024 \mathrm{mmol}), \mathrm{NaHCO}_{3}(99 \mathrm{mg}$, $1.18 \mathrm{mmol})$ and Oxone ${ }^{\circledR}(1.45 \mathrm{~g}, 2.36 \mathrm{mmol})$ were used in this transformation. Purification of the product by flash chromatography $\left(\mathrm{SiO}_{2}\right.$, hexanes:EtOAc, 2:1) provided compound 24 as a $1: 1$ 
mixture of regioisomers $(62 \mathrm{mg}, 66 \%)$. IR $\left(\mathrm{CH}_{2} \mathrm{Cl}_{2}\right.$ cast $) 3351,2944,2859,1705,1260 \mathrm{~cm}^{-1} ;{ }^{1} \mathbf{H}-$ NMR $\left(\mathrm{CDCl}_{3}, 400 \mathrm{MHz}\right) \delta 4.88(1 \mathrm{H}, \mathrm{m}), 3.80(3 \mathrm{H}, \mathrm{s}), 2.80(1 \mathrm{H}, \mathrm{m}), 1.96-1.62(4 \mathrm{H}, \mathrm{m}), 1.50-$ 1.19 (4H, m), 1.50-1.19 (6H, m); ${ }^{13} \mathbf{C}-\mathbf{N M R}\left(\mathrm{CDCl}_{3}, 100 \mathrm{MHz}\right) \delta 207.0,168.8,76.4,53.3,46.8$, 29.5, 28.0, 25.9; HRMS (EI) Calc'd for [M+] $\mathrm{C}_{10} \mathrm{H}_{16} \mathrm{O}_{4} 200.1049$, found 200.1049.

\section{Isomerization Control Experiments}<smiles>COC(=O)C(O)C(O)C1CCCCC1</smiles>

To a stirring solution of 3-cyclohexyl-2-hydroxy-3-oxo-propionic acid methyl ester (24) (11 $\mathrm{mg}, 0.055 \mathrm{mmol})$ in a 6:6:1 mixture of MeCN:EtOAc: $\mathrm{H}_{2} \mathrm{O}(1.3 \mathrm{~mL})$ was added Grubbs' catalyst 2 (2.5 mg, $0.003 \mathrm{mmol}), \mathrm{NaHCO}_{3}(12 \mathrm{mg}, 0.14 \mathrm{mmol})$ and Oxone ${ }^{\circledR}(169 \mathrm{mg}, 0.28 \mathrm{mmol})$ at 0 ${ }^{\circ} \mathrm{C}$. The resulting reaction mixture was stirred at $0{ }^{\circ} \mathrm{C}$ for $1 \mathrm{~h}$, at which time the mixture was diluted with EtOAc $(2.0 \mathrm{~mL})$ and filtered. The filtrate was washed with a saturated solution of $\mathrm{Na}_{2} \mathrm{SO}_{3}$ and dried over $\mathrm{Na}_{2} \mathrm{SO}_{4}$. Filtration and concentration in vacuo gave an amber oil. ${ }^{1} H$-NMR analysis of the reaction mixture indicated a nearly 1:1 ratio of 3-cyclohexyl-2-hydroxy3-oxo-propionic acid methyl ester (24) to its regioisomer 3-cyclohexyl-3-hydroxy-2-oxopropionic acid methyl ester.<smiles>COC(=O)C(=O)C(O)C1CCCCC1</smiles>

To a stirring solution of 3-cyclohexyl-3-hydroxy-2-oxo-propionic acid methyl ester (16 mg, $0.08 \mathrm{mmol})$ in a 6:6:1 mixture of MeCN:EtOAc: $\mathrm{H}_{2} \mathrm{O}(1.3 \mathrm{~mL})$ was added Grubbs' catalyst 2 (3.5 $\mathrm{mg}, 0.004 \mathrm{mmol}), \mathrm{NaHCO}_{3}(17 \mathrm{mg}, 0.20 \mathrm{mmol})$ and Oxone ${ }^{\circledR}(246 \mathrm{mg}, 0.40 \mathrm{mmol})$ at $0{ }^{\circ} \mathrm{C}$. The resulting reaction mixture was stirred at $0{ }^{\circ} \mathrm{C}$ for $1 \mathrm{~h}$, at which time the mixture was diluted with EtOAc $(2.0 \mathrm{~mL})$ and filtered. The filtrate was washed with a saturated solution of $\mathrm{Na}_{2} \mathrm{SO}_{3}$ and dried over $\mathrm{Na}_{2} \mathrm{SO}_{4}$. Filtration and concentration in vacuo gave an amber oil. ${ }^{1} \mathrm{H}-\mathrm{NMR}$ analysis of the reaction mixture indicated that no isomerization had occurred. These observations suggest the results obtained are a non-thermodynamic ratio of the two diketohydroxides.<smiles>C=CC(=O)OCC(C)COC(C)=O</smiles>

\section{7-Acetoxy-2-hydroxy-3-oxo-heptanoic acid acid methyl ester (26).}

General procedure $\mathbf{B}$ was followed. 1-Hexen-6-yl acetate (25) (43 mg, $0.30 \mathrm{mmol})$, methylacrylate (23) $(55 \mu \mathrm{L}, 0.60 \mathrm{mmol})$, Grubbs' catalyst 2 (13 mg, $0.015 \mathrm{mmol}), \mathrm{NaHCO}_{3}(63 \mathrm{mg}$, $0.76 \mathrm{mmol})$ and Oxone ${ }^{\circledR}(928 \mathrm{mg}, 1.51 \mathrm{mmol})$ were used in this transformation. Purification of the product by flash chromatography $\left(\mathrm{SiO}_{2}\right.$, hexanes:EtOAc, 2:1) provided compound 26 as a 1.5:1 mixture of regioisomers $(39 \mathrm{mg}, 56 \%)$. IR $\left(\mathrm{CH}_{2} \mathrm{Cl}_{2}\right.$ cast $) 3428,2959,7132,1255 \mathrm{~cm}^{-1}$; ${ }^{1}$ H-NMR $\left(\mathrm{CDCl}_{3}, 400 \mathrm{MHz}\right) \delta 4.95(2 \mathrm{H}, \mathrm{m}), 4.04(2 \mathrm{H}, \mathrm{t}, J=6.8 \mathrm{~Hz}), 3.84(3 \mathrm{H}, \mathrm{s}), 2.62(2 \mathrm{H}, \mathrm{t}, J$ $=6.8 \mathrm{~Hz}), 2.02(3 \mathrm{H}, \mathrm{s}), 1.74-1.58(4 \mathrm{H}, \mathrm{m}) ;{ }^{13} \mathbf{C}-\mathbf{N M R}\left(\mathrm{CDCl}_{3}, 100 \mathrm{MHz}\right) \delta 202.7,171.1,169.4$, 92.6, 64.0, 54.1, 35.4, 28.0, 21.2, 20.1; HRMS (EI) calc'd for [M+] $\mathrm{C}_{10} \mathrm{H}_{16} \mathrm{O}_{6} 232.0947$, found 232.0955 . 
<smiles>C=CC(=O)OC</smiles>

\section{2-Hydroxy-3-oxo-3-phenyl-propionic acid methyl ester (28). ${ }^{2}$}

General procedure B was followed. Styrene (27) $(58 \mathrm{mg}, 0.58 \mathrm{mmol})$, methylacrylate (23) (100 $\mu \mathrm{L}, 1.11 \mathrm{mmol})$, Grubbs' catalyst 2 (24 mg, $0.028 \mathrm{mmol}), \mathrm{NaHCO}_{3}(117 \mathrm{mg}, 1.39 \mathrm{mmol})$ and Oxone ${ }^{\circledR}(1.71 \mathrm{~g}, 2.79 \mathrm{mmol})$ were used in this transformation. Purification of the product by flash chromatography $\left(\mathrm{SiO}_{2}\right.$, hexanes:EtOAc, 2:1) provided compound $\mathbf{2 8}$ as a 2:1 mixture of regioisomers (60 mg, 56\%). IR $\left(\mathrm{CH}_{2} \mathrm{Cl}_{2}\right.$ cast) 3412, 3060, 3026, 2926, 1746, 1687, 1291, 1238, $1128 \mathrm{~cm}^{-1} ;{ }^{1} \mathbf{H}-\mathbf{N M R}\left(\mathrm{CDCl}_{3}, 400 \mathrm{MHz}\right) \delta 8.07(2 \mathrm{H}, \mathrm{m}), 7.64(1 \mathrm{H}, \mathrm{m}), 7.50(2 \mathrm{H}, \mathrm{m}), 5.60(1 \mathrm{H}$, m), 4.27 (1H, bs), $3.71(3 \mathrm{H}, \mathrm{s}) ;{ }^{13} \mathbf{C}-\mathbf{N M R}\left(\mathrm{CDCl}_{3}, 100 \mathrm{MHz}\right) \delta 193.5,169.1,134.8,129.5$, 128.9, 74.6, 53.4; HRMS (EI) calc'd for [M+] $\mathrm{C}_{10} \mathrm{H}_{10} \mathrm{O}_{4}$ 194.0579, found 194.0580.

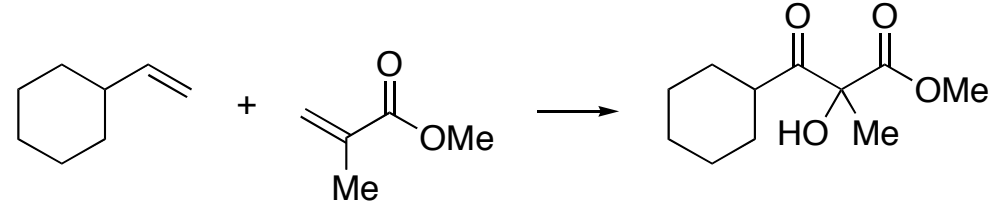

3-Cyclohexyl-2-hydroxy-2-methyl-3-oxo-propionic acid methyl ester (30). ${ }^{2,3}$

General procedure B was followed. Vinylcyclohexane (22) (63 mg, $0.57 \mathrm{mmol})$, methyl methacrylate (29) $(122 \mu \mathrm{L}, 1.14 \mathrm{mmol})$, Grubbs' catalyst 2 (24 mg, $0.029 \mathrm{mmol}), \mathrm{NaHCO}_{3}(120$ $\mathrm{mg}, 1.43 \mathrm{mmol})$ and Oxone ${ }^{\circledR}(1.76 \mathrm{~g}, 2.86 \mathrm{mmol})$ were used in this transformation. Purification of the product by flash chromatography $\left(\mathrm{SiO}_{2}\right.$, hexanes:EtOAc, 2:1) provided compound 30 as a colorless oil (60 mg, 49\%). IR ( $\mathrm{CH}_{2} \mathrm{Cl}_{2}$ cast) 3476, 2946, 2897, 2859, 1743, 1719, 1264, 1148 $\mathrm{cm}^{-1}$; ${ }^{1} \mathbf{H}-\mathbf{N M R}\left(\mathrm{CDCl}_{3}, 400 \mathrm{MHz}\right) \delta 4.22(1 \mathrm{H}, \mathrm{s}) 3.76(1 \mathrm{H}, \mathrm{s}), 2.83(1 \mathrm{H}, \mathrm{tt}, J=11.2,3.2 \mathrm{~Hz})$, 1.79-1.59 (5H, m), $1.56(3 \mathrm{H}, \mathrm{s}), 1.42-1.31(2 \mathrm{H}, \mathrm{m}), 1.29-1.17(3 \mathrm{H}, \mathrm{m}) ;{ }^{13} \mathbf{C}-\mathbf{N M R}\left(\mathrm{CDCl}_{3}, 100\right.$ MHz) $\delta 209.9,171.8,81.0,53.4,45.3,29.8,29.7,25.8,22.1$; HRMS (ESI) Calc'd for [M+Na] ${ }^{+}$ $\mathrm{C}_{11} \mathrm{H}_{18} \mathrm{O}_{4} \mathrm{Na} 237.1103$, found 237.1109.<smiles>C=C[C+]=COC(C)=O</smiles>

\section{7-Acetoxy-2-hydroxy-2-methyl-3-oxo-heptanoic acid acid methyl ester (31).}

General procedure B was followed. 1-Hexen-6-yl acetate (25) (59 mg, $0.42 \mathrm{mmol})$, methyl methacrylate (29) $(89 \mu \mathrm{L}, 0.83 \mathrm{mmol})$, Grubbs' catalyst 2 (18 mg, $0.021 \mathrm{mmol}), \mathrm{NaHCO}_{3}(87$ $\mathrm{mg}, 1.04 \mathrm{mmol})$ and Oxone ${ }^{\circledR}(1.28 \mathrm{~g}, 2.08 \mathrm{mmol})$ were used in this transformation. Purification of the product by flash chromatography $\left(\mathrm{SiO}_{2}\right.$, hexanes:EtOAc, 2:1) provided compound 31 as a colorless oil $(78 \mathrm{mg}, 76 \%)$. IR $\left(\mathrm{CH}_{2} \mathrm{Cl}_{2}\right.$ cast) 3466, 2956, 1745, 1738, 1369, $1243 \mathrm{~cm}^{-1}$; ${ }^{1}$ H-NMR $\left(\mathrm{CDCl}_{3}, 400 \mathrm{MHz}\right) \delta 4.20(1 \mathrm{H}, \mathrm{bs}), 4.00(2 \mathrm{H}, \mathrm{t}, J=6 \mathrm{~Hz}), 3.74(3 \mathrm{H}, \mathrm{s}), 2.60(2 \mathrm{H}, \mathrm{m})$, $1.98(3 \mathrm{H}, \mathrm{s}), 1.67-1.50(7 \mathrm{H}, \mathrm{m}) ;{ }^{13} \mathbf{C}-\mathbf{N M R}\left(\mathrm{CDCl}_{3}, 100 \mathrm{MHz}\right) \delta 206.5,171.9,171.1,81.0,64.1$, 53.6, 36.0, 28.1, 22.3, 20.3; HRMS (ESI) Calc'd for $[\mathrm{M}+\mathrm{Na}]^{+} \mathrm{C}_{11} \mathrm{H}_{18} \mathrm{O}_{6} \mathrm{Na} 269.1001$, found 269.0988 . 


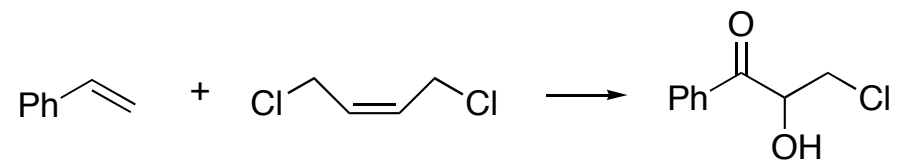

\section{3-Chloro-2-hydroxy-1-phenyl-propan-1-one (33). ${ }^{4}$}

General procedure B was followed. Styrene (27) $(63 \mathrm{mg}, 0.61 \mathrm{mmol})$, cis-1,4-dichloro-2butene (32) $(127 \mu \mathrm{L}, 1.21 \mathrm{mmol})$, Grubbs' catalyst $2(51 \mathrm{mg}, 0.061 \mathrm{mmol}), \mathrm{NaHCO}_{3}(127 \mathrm{mg}$, $1.51 \mathrm{mmol})$ and Oxone ${ }^{\circledR}(1.86 \mathrm{~g}, 3.03 \mathrm{mmol})$ were used in this transformation. Purification of the product by flash chromatography $\left(\mathrm{SiO}_{2}\right.$, hexanes:EtOAc, $\left.2: 1\right)$ provided compound $\mathbf{3 3}$ as a colorless oil $(53 \mathrm{mg}, 47 \%)$. IR $\left(\mathrm{CH}_{2} \mathrm{Cl}_{2}\right.$ cast $) 3434,3060,3029,1712,1697,1289,1165 \mathrm{~cm}^{-1}$; ${ }^{1}$ H-NMR $\left(\mathrm{CDCl}_{3}, 400 \mathrm{MHz}\right) \delta 7.89(2 \mathrm{H}, \mathrm{m}), 7.64(1 \mathrm{H}, \mathrm{m}), 7.51(2 \mathrm{H}, \mathrm{m}), 5.34(1 \mathrm{H}, \mathrm{bs}), 4.10$ $(1 \mathrm{H}, \mathrm{m}), 3.93(1 \mathrm{H}, \mathrm{dd}, J=11.6,3.5 \mathrm{~Hz}), 3.75(1 \mathrm{H}, \mathrm{dd}, J=11.6,4.0 \mathrm{~Hz}) ;{ }^{13} \mathbf{C}-\mathbf{N M R}\left(\mathrm{CDCl}_{3}, 100\right.$ MHz) $\delta 197.8,134.5,130.2,129.2,128.6,72.8,47.6$; HRMS (EI) Calc'd for [M+] $\mathrm{C}_{9} \mathrm{H}_{9} \mathrm{ClO}_{2}$ 184.0291, found 184.0283 .

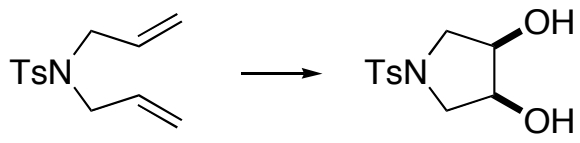

\section{(3S, 4R)-1-Toluene-4-sulfonyl)-pyrrol-3,4-diol (35). ${ }^{5}$}

General procedure $\mathbf{C}$ was followed. $N, N$-Diallyl-4-methyl-benzenesulfonamide (34) $(80 \mathrm{mg}$, $0.32 \mathrm{mmol})$, Grubbs' catalyst 2 (14 mg, $0.016 \mathrm{mmol}), \mathrm{NaIO}_{4}(102 \mathrm{mg}, 0.48 \mathrm{mmol})$ and $\mathrm{CeCl}_{3} \cdot 7 \mathrm{H}_{2} \mathrm{O}(12 \mathrm{mg}, 0.032 \mathrm{mmol})$ were used in this transformation. Purification of the product by flash chromatography ( $\mathrm{SiO}_{2}$, hexanes:EtOAc, 1:4) provided diol $\mathbf{3 5}$ as an amber oil (38 $\mathrm{mg}$, 63\%). IR $\left(\mathrm{CH}_{2} \mathrm{Cl}_{2}\right.$ cast $) 3489,3409,2928,2909 \mathrm{~cm}^{-1} ;{ }^{1} \mathbf{H}-\mathbf{N M R}\left(\mathrm{CDCl}_{3}, 300 \mathrm{MHz}\right) \delta 7.68(2 \mathrm{H}$, m), $7.31(2 \mathrm{H}, \mathrm{m}), 4.12(2 \mathrm{H}, \mathrm{m}), 3.48(2 \mathrm{H}, \mathrm{dd}, J=10.6,5.7 \mathrm{~Hz}), 3.18(2 \mathrm{H}, \mathrm{dd}, J=10.6,3.8 \mathrm{~Hz})$, 2.89 (2H, bs), $2.42(3 \mathrm{H}, \mathrm{s}) ;{ }^{13} \mathbf{C}-\mathbf{N M R}\left(\mathrm{CDCl}_{3}, 100 \mathrm{MHz}\right) \delta 130.1,129.8,127.8,127.6,70.9$, 52.4, 21.9; HRMS (ESI) Calc'd for $[\mathrm{M}+\mathrm{Na}]^{+} \mathrm{C}_{11} \mathrm{H}_{15} \mathrm{NO}_{4} \mathrm{NaS} 280.0619$, found 280.0616.

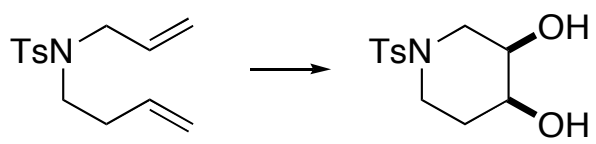

(3S, 4R)-1-(Toluene-4-sulfonyl)-piperidine-3,4-diol (37). ${ }^{5}$

General procedure $\mathbf{C}$ was followed. $N$-Allyl- $N$-but-3-enyl-4-methyl-benzenesulfonamide (36) $(50 \mathrm{mg}, 0.19 \mathrm{mmol})$, Grubbs' catalyst 2 (8 mg, $0.009 \mathrm{mmol}), \mathrm{NaIO}_{4}(61 \mathrm{mg}, 0.285 \mathrm{mmol})$ and $\mathrm{CeCl}_{3} \cdot 7 \mathrm{H}_{2} \mathrm{O}(7 \mathrm{mg}, 0.02 \mathrm{mmol})$ were used in this transformation. Purification of the product by flash chromatography $\left(\mathrm{SiO}_{2}\right.$, hexanes:EtOAc, 1:4) provided diol 37 as an amber oil (35 mg, 69\%). IR $\left(\mathrm{CH}_{2} \mathrm{Cl}_{2}\right.$ cast $) 3371,3060,3028,1336,1162 \mathrm{~cm}^{-1} ;{ }^{1} \mathbf{H}-\mathbf{N M R}\left(\mathrm{CDCl}_{3}, 400 \mathrm{MHz}\right) \delta 7.63$ $(2 \mathrm{H}, \mathrm{m}), 7.31(2 \mathrm{H}, \mathrm{m}), 3.86(1 \mathrm{H}, \mathrm{bs}), 3.75(1 \mathrm{H}, \mathrm{bs}), 3.15(1 \mathrm{H}, \mathrm{m}), 3.01(3 \mathrm{H}, \mathrm{m}), 2.41(3 \mathrm{H}, \mathrm{s})$, $2.20(1 \mathrm{H}, \mathrm{m}), 2.09(1 \mathrm{H}, \mathrm{m}), 1.84(2 \mathrm{H}, \mathrm{m}) ;{ }^{13} \mathbf{C}-\mathbf{N M R}\left(\mathrm{CDCl}_{3}, 100 \mathrm{MHz}\right) \delta 143.9,133.1,129.9$, 127.7, 68.0, 67.7, 48.1, 42.2, 29.7, 21.9; HRMS (ESI) Calc'd for $[\mathrm{M}+\mathrm{Na}]^{+} \mathrm{C}_{12} \mathrm{H}_{17} \mathrm{NO}_{4} \mathrm{NaS}$ 294.0776, found 294.0786. 


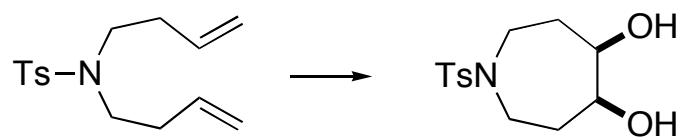

(3S, 4R)-3,4-Dihydroxycyclohexane-1,1-dicarboxylic acid diethyl ester (38). ${ }^{5}$

General procedure $\mathbf{C}$ was followed. $N, N$-Dibut-3-enyl-4-methyl-benzenesulfonamide (6) (54 $\mathrm{mg}, 0.19 \mathrm{mmol})$, Grubbs' catalyst 2 (8 mg, $0.0097 \mathrm{mmol}), \mathrm{NaIO}_{4}(61 \mathrm{mg}, 0.29 \mathrm{mmol})$ and $\mathrm{CeCl}_{3} \cdot 7 \mathrm{H}_{2} \mathrm{O}(7 \mathrm{mg}, 0.019 \mathrm{mmol})$ were used in this transformation. Purification of the product by flash chromatography $\left(\mathrm{SiO}_{2}\right.$, hexanes:EtOAc, 1:4) provided diol 38 as an amber oil (38 mg, 65\%). IR $\left(\mathrm{CH}_{2} \mathrm{Cl}_{2}\right.$ cast $) 3433,3015,2920,1643,1160 \mathrm{~cm}^{-1} ;{ }^{1} \mathbf{H}-\mathbf{N M R}\left(\mathrm{CDCl}_{3}, 400 \mathrm{MHz}\right) \delta 7.66$ $(2 \mathrm{H}, \mathrm{m}), 7.30(2 \mathrm{H}, \mathrm{m}), 3.96(2 \mathrm{H}, \mathrm{d}, J=7.6 \mathrm{~Hz}), 3.43(2 \mathrm{H}, \mathrm{ddd}, J=12.8,8.0,4.0 \mathrm{~Hz}), 3.18(2 \mathrm{H}$, $\mathrm{ddd}, J=11.2,7.6,3.6 \mathrm{~Hz}), 2.42(3 \mathrm{H}, \mathrm{s}), 2.17(2 \mathrm{H}, \mathrm{bs}), 2.06(2 \mathrm{H}, \mathrm{m}), 1.80(2 \mathrm{H}, \mathrm{m})$; ${ }^{13} \mathbf{C}-\mathbf{N M R}$ $\left(\mathrm{CDCl}_{3}, 100 \mathrm{MHz}\right) \delta 143.4,135.7,129.8,127.0,72.5,42.4,31.3,21.8$; HRMS (ESI) Calc'd for $[\mathrm{M}+\mathrm{Na}]^{+} \mathrm{C}_{13} \mathrm{H}_{19} \mathrm{NO}_{4} \mathrm{SNa} 308.0932$, found 308.0923.

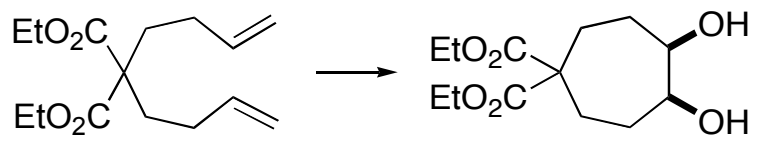

(4S, 5R)-4,5-Dihydroxycycloheptane-1,1-dicarboxylic acid diethyl ester (39).

General procedure $\mathbf{C}$ was followed. 2-Allyl-2-but-3-enyl-malonic acid diethyl ester (8) 60 $\mathrm{mg}, 0.19 \mathrm{mmol})$, Grubbs' catalyst $2(10 \mathrm{mg}, 0.011 \mathrm{mmol}), \mathrm{NaIO}_{4}(72 \mathrm{mg}, 0.34 \mathrm{mmol})$ and $\mathrm{CeCl}_{3} \cdot 7 \mathrm{H}_{2} \mathrm{O}(8 \mathrm{mg}, 0.022 \mathrm{mmol})$ were used in this transformation. Purification of the product by flash chromatography $\left(\mathrm{SiO}_{2}\right.$, hexanes:EtOAc, 1:4) provided diol 39 as an amber oil (41 mg, 67\%). IR $\left(\mathrm{CH}_{2} \mathrm{Cl}_{2}\right.$ cast $) 3409,2961,1727 \mathrm{~cm}^{-1} ;{ }^{1} \mathbf{H}-\mathbf{N M R}\left(\mathrm{CDCl}_{3}, 400 \mathrm{MHz}\right) \delta 4.14(4 \mathrm{H}, \mathrm{m})$, $3.76(2 \mathrm{H}, \mathrm{m}), 2.34(2 \mathrm{H}, \mathrm{m}), 2.26(2 \mathrm{H}, \mathrm{bs}), 1.85(2 \mathrm{H}, \mathrm{m}), 1.62(2 \mathrm{H}, \mathrm{t}, J=13.2 \mathrm{~Hz}), 1.22(6 \mathrm{H}, \mathrm{m})$; ${ }^{13} \mathbf{C}-\mathbf{N M R}\left(\mathrm{CDCl}_{3}, 100 \mathrm{MHz}\right) \delta 172.6,73.1,61.6,61.5,56.7,34.1,26.9,25.9,14.3$; HRMS (ESI) Calc'd for $[\mathrm{M}+\mathrm{Na}]^{+} \mathrm{C}_{13} \mathrm{H}_{22} \mathrm{O}_{6} \mathrm{Na} 297.1314$, found 297.1311.<smiles>C=CCCCC(C=C)OC(C)=O</smiles>

\section{$(1 S, 2 S, 3 S)-2,3-D i h y d r o x y c y c l o h e x y l a c e t a t e(41) .^{5,6}$}

General procedure $\mathbf{C}$ was followed. Acetic acid 1-vinyl-hex-5-enyl ester (40) (65 mg, 0.39 mmol), Grubbs' catalyst 2 (16 mg, $0.019 \mathrm{mmol}), \mathrm{NaIO}_{4}(124 \mathrm{mg}, 0.58 \mathrm{mmol})$ and $\mathrm{CeCl}_{3} \cdot 7 \mathrm{H}_{2} \mathrm{O}$ $(15 \mathrm{mg}, 0.04 \mathrm{mmol}$ ) were used in this transformation. Purification of the product by flash chromatography $\left(\mathrm{SiO}_{2}\right.$, hexanes:EtOAc, 1:4) provided diol 41 as a 22:1 mixture of diastereomers (40 mg, 60\%). IR $\left(\mathrm{CH}_{2} \mathrm{Cl}_{2}\right.$ cast) 3408, 2949, 2871, 1721, 1376, $1262 \mathrm{~cm}^{-1} ;{ }^{1} \mathbf{H}-\mathbf{N M R}\left(\mathrm{CDCl}_{3}\right.$, $300 \mathrm{MHz}) \delta 4.98(1 \mathrm{H}, \mathrm{m}), 4.08(1 \mathrm{H}, \mathrm{m}), 3.57(1 \mathrm{H}, \mathrm{dd}, J=8.9,2.3 \mathrm{~Hz}), 3.02(1 \mathrm{H}, \mathrm{bs}), 2.57(1 \mathrm{H}$, bs), $2.09(3 \mathrm{H}, \mathrm{s}), 2.01-1.84(2 \mathrm{H}, \mathrm{m}), 1.77-1.66(1 \mathrm{H}, \mathrm{m}), 1.57-1.26(3 \mathrm{H}, \mathrm{m}) ;{ }^{13} \mathrm{C}-\mathrm{NMR}\left(\mathrm{CDCl}_{3}\right.$, $100 \mathrm{MHz}) \delta 171.8,74.5,74.3,70.1,30.1,29.4,21.6,18.4$; HRMS (ESI) calc'd for $[\mathrm{M}+\mathrm{Na}]^{+}$ $\mathrm{C}_{8} \mathrm{H}_{14} \mathrm{O}_{4} \mathrm{Na}$ 197.0790, for 197.0787. 
<smiles>C=CCCCC(C=C)OCCCCCCCC(=O)OC(C)(C)C</smiles>

\section{(1S, 2S, 3S)-2,3-Dihydroxycycloxylbenzoate (42). ${ }^{7}$}

General procedure $\mathbf{C}$ was followed. Benzoic acid 1-vinyl-hex-5-enyl ester (20) (59 mg, 0.26 mmol), Grubbs' catalyst 2 (11 mg, $0.013 \mathrm{mmol}), \mathrm{NaIO}_{4}(81 \mathrm{mg}, 0.38 \mathrm{mmol})$ and $\mathrm{CeCl}_{3} \cdot 7 \mathrm{H}_{2} \mathrm{O}$ $(10 \mathrm{mg}, 0.026 \mathrm{mmol})$ were used in this transformation. Purification of the product by flash chromatography $\left(\mathrm{SiO}_{2}\right.$, hexanes:EtOAc, 1:4) provided diol 42 as an amber oil (48 $\left.\mathrm{mg}, 81 \%\right)$. IR $\left(\mathrm{CH}_{2} \mathrm{Cl}_{2}\right.$ cast $) 3390,3026,2924,1712,1602,1276 \mathrm{~cm}^{-1} ;{ }^{1} \mathbf{H}-\mathbf{N M R} \delta 8.01(2 \mathrm{H}, \mathrm{m}), 7.53(1 \mathrm{H}, \mathrm{m})$, $7.40(2 \mathrm{H}, \mathrm{m}), 5.24(1 \mathrm{H}, \mathrm{m}), 4.09(2 \mathrm{H}, \mathrm{m}), 3.72(1 \mathrm{H}, \mathrm{dd}, J=8.2,2.8 \mathrm{~Hz}), 3.52(1 \mathrm{H}, \mathrm{bs}), 3.08(1 \mathrm{H}$, bs), $2.02(1 \mathrm{H}, \mathrm{m}), 1.87(1 \mathrm{H}, \mathrm{m}), 1.73(1 \mathrm{H}, \mathrm{m}) ;{ }^{13} \mathbf{C}-\mathbf{N M R}\left(\mathrm{CDCl}_{3}, 100 \mathrm{MHz}\right) \delta 170.0,133.2$, 130.2, 129.7, 128.4, 75.0, 74.1, 70.1, 30.0, 29.1, 18.5; HRMS (ESI) Calc'd for [M+Na] ${ }^{+}$ $\mathrm{C}_{13} \mathrm{H}_{16} \mathrm{O}_{4} \mathrm{Na} 259.0946$, found 259.0938.<smiles>C=CCOC(CC=C)C1CCCCC1</smiles>

\section{$(3 R, 4 S, 6 R)-6-C y c l o h e x y l-t e t r a h y d r o-p y r a n-3,4-d i o l ~(44)$.}

General procedure $\mathbf{C}$ was followed. (1-Allyloxy-but-3-enyl)-cyclohexane (43) (57 mg, 0.29 mmol), Grubbs' catalyst 2 (13 mg, $0.015 \mathrm{mmol}), \mathrm{NaIO}_{4}(93 \mathrm{mg}, 0.44 \mathrm{mmol})$ and $\mathrm{CeCl}_{3} \cdot 7 \mathrm{H}_{2} \mathrm{O}$ (11 $\mathrm{mg}, 0.029 \mathrm{mmol}$ ) were used in this transformation. Purification of the product by flash chromatography $\left(\mathrm{SiO}_{2}\right.$, hexanes:EtOAc, 1:4) provided diol 44 as an 3:2 mixture of diastereomers (40 mg, 69\%). IR $\left(\mathrm{CH}_{2} \mathrm{Cl}_{2}\right.$ cast) $3408,3316,2915,2857,1244 \mathrm{~cm}^{-1} ;{ }^{1} \mathbf{H}-\mathbf{N M R}\left(\mathrm{CDCl}_{3}, 400\right.$ MHz) $\delta 4.03(1 \mathrm{H}, \mathrm{dd}, J=12.5,2.2 \mathrm{~Hz}), 3.73(2 \mathrm{H}, \mathrm{m}), 3.46(1 \mathrm{H}, \mathrm{dd}, J=12.5,0.9 \mathrm{~Hz}), 3.00(1 \mathrm{H}$, ddd, $J=11.7,6.8,2.2 \mathrm{~Hz}), 2.17(2 \mathrm{H}, \mathrm{m}), 1.94-1.159(7 \mathrm{H}, \mathrm{m}), 1.44(1 \mathrm{H}, \mathrm{m}), 2.21(3 \mathrm{H}, \mathrm{m})$; ${ }^{13}$ C-NMR $\left(\mathrm{CDCl}_{3}, 100 \mathrm{MHz}\right) \delta 81.1,70.7,69.5,68.6,42.8,32.9,29.3,28.8,26.8,26.4,26.3$; HRMS (ESI) Calc'd for [M+Na] ${ }^{+} \mathrm{C}_{11} \mathrm{H}_{20} \mathrm{O}_{3} \mathrm{Na} 223.1310$, found 223.1321.<smiles>C=CCC([Al])N(CC=C)c1ccc(OC)cc1</smiles>

\section{(3R, 4S, 6R)-6-(4-Methoxy-phenyl)-1-(toluene-4-sulfonyl)-piperidine-3,4-diol (46).}

General procedure $\mathbf{C}$ was followed. $N$-Allyl- $N$-[1-(4-methoxy-phenyl)-but-3-enyl]-4-methylbenzenesulfonamide (45) $(51 \mathrm{mg}, 0.14 \mathrm{mmol})$, Grubbs' catalyst 2 (6 mg, $0.007 \mathrm{mmol}), \mathrm{NaIO}_{4}$ (44 mg, $0.206 \mathrm{mmol}$ ) and $\mathrm{CeCl}_{3} \cdot 7 \mathrm{H}_{2} \mathrm{O}(5 \mathrm{mg}, 0.014 \mathrm{mmol}$ ) were used in this transformation. Purification of the product by flash chromatography $\left(\mathrm{SiO}_{2}\right.$, hexanes:EtOAc, 1:4) provided diol 46 as an 7:1 mixture of diastereomers (40 mg, 77\%). IR $\left(\mathrm{CH}_{2} \mathrm{Cl}_{2}\right.$ cast) $3424,3032,2928,2899$, 1610, 1513, 1325, 1251, $1157 \mathrm{~cm}^{-1}$; ${ }^{1} \mathbf{H}-\mathbf{N M R}\left(\mathrm{CDCl}_{3}, 500 \mathrm{MHz}\right) \delta 7.83(2 \mathrm{H}, \mathrm{m}), 7.33(2 \mathrm{H}, \mathrm{m})$, $7.19(2 \mathrm{H}, \mathrm{m}), 6.85(2 \mathrm{H}, \mathrm{m}), 5.35(1 \mathrm{H}, \mathrm{m}), 3.97(1 \mathrm{H}, \mathrm{dt}, J=15.2,2.1 \mathrm{~Hz}), 3.81(3 \mathrm{H}, \mathrm{s}), 3.74(1 \mathrm{H}$, bs), $3.66(1 \mathrm{H}, \mathrm{m}), 3.11(1 \mathrm{H}, \mathrm{dd}, J=15.6,1.6 \mathrm{~Hz}), 2.52(1 \mathrm{H}, \mathrm{d}, J=5.2 \mathrm{~Hz}), 2.46(3 \mathrm{H}, \mathrm{s}), 2.37$ 
$(1 \mathrm{H}, \mathrm{m}), 2.15(1 \mathrm{H}, \mathrm{d}, J=8.2 \mathrm{~Hz}), 2.04(1 \mathrm{H}, \mathrm{m}) ;{ }^{13} \mathbf{C}-\mathbf{N M R}\left(\mathrm{CDCl}_{3}, 100 \mathrm{MHz}\right) \delta 158.8,143.5$, 129.6, 129.1, 127.7, 127.6, 114.3, 67.3, 66.0, 55.5, 55.1, 45.9, 30.7, 21.9; HRMS (ESI) Calc'd for $[\mathrm{M}+\mathrm{Na}]^{+} \mathrm{C}_{19} \mathrm{H}_{23} \mathrm{NO}_{5} \mathrm{SNa} 400.1195$, found 400.1185 .<smiles>C=CC(=O)OC</smiles>

(2S, 3R)-3-Cyclohexyl-2,3-dihydroxypropanoic acid methyl ester (47). ${ }^{8}$

General procedure D was followed. Vinylcyclohexane (22) (68 mg, $0.62 \mathrm{mmol})$, methylacrylate $(\mathbf{2 3})(111 \mu \mathrm{L}, 1.23 \mathrm{mmol})$, Grubbs' catalyst 2 (26 mg, $0.031 \mathrm{mmol}), \mathrm{NaIO}_{4}(198 \mathrm{mg}$, $0.93 \mathrm{mmol})$ and $\mathrm{CeCl}_{3} \cdot 7 \mathrm{H}_{2} \mathrm{O}(23 \mathrm{mg}, 0.062 \mathrm{mmol})$ were used in this transformation. Purification of the product by flash chromatography $\left(\mathrm{SiO}_{2}\right.$, hexanes:EtOAc, 2:1) provided diol 47 as an amber oil (90 mg, 77\%). IR $\left(\mathrm{CH}_{2} \mathrm{Cl}_{2}\right.$ cast) 3414, 2948, 2897, 2855, 1741, 1441, 1278, 1225, $1118 \mathrm{~cm}^{-1} ;{ }^{1} \mathbf{H}-\mathbf{N M R}\left(\mathrm{CDCl}_{3}\right) \delta 4.29(1 \mathrm{H}, \mathrm{m}), 3.81(3 \mathrm{H}, \mathrm{s}), 3.54(1 \mathrm{H}, \mathrm{m}), 3.00$ (1H, m), 2.01 $(1 \mathrm{H}, \mathrm{m}), 1.81\left(1.59(6 \mathrm{H}, \mathrm{m}), 1.28-0.96(4 \mathrm{H}, \mathrm{m}) ;{ }^{13} \mathbf{C}-\mathbf{N M R}\left(\mathrm{CDCl}_{3}, 100 \mathrm{MHz}\right) \delta 174.7,71.1\right.$, 53.0, 40.6, 29.6, 29.4, 26.6, 26.2; HRMS (ESI) Calc'd for [M+Na] ${ }^{+} \mathrm{C}_{10} \mathrm{H}_{18} \mathrm{O}_{4} \mathrm{Na} 225.1103$, found 225.1092 .

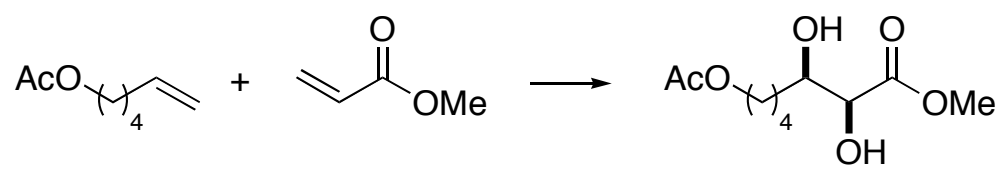

(2S, 3R)-7-Acetoxy-2,3-dihydroxyheptanoic acid acid methyl ester (48).

General procedure D was followed. 1-Hexen-6-yl acetate $(\mathbf{2 5})(61 \mathrm{mg}, 0.43 \mathrm{mmol})$, methylacrylate (23) $(78 \mu \mathrm{L}, 0.86 \mathrm{mmol})$, Grubbs' catalyst 2 (18 mg, $0.022 \mathrm{mmol}), \mathrm{NaIO}_{4}(138 \mathrm{mg}, 0.64$ $\mathrm{mmol})$ and $\mathrm{CeCl}_{3} \cdot 7 \mathrm{H}_{2} \mathrm{O}(16 \mathrm{mg}, 0.043 \mathrm{mmol})$ were used in this transformation. Purification of the product by flash chromatography $\left(\mathrm{SiO}_{2}\right.$, hexanes:EtOAc, 1:1) provided diol 48 as an amber oil $(56 \mathrm{mg}, 56 \%)$. IR $\left(\mathrm{CH}_{2} \mathrm{Cl}_{2}\right.$ cast $) 3536,2953,2915,1738,1248 \mathrm{~cm}^{-1} ;{ }^{1} \mathbf{H}-\mathbf{N M R}\left(\mathrm{CDCl}_{3}, 300\right.$ $\mathrm{MHz}) \delta 4.08(3 \mathrm{H}, \mathrm{m}), 3.84(4 \mathrm{H}, \mathrm{m}), 3.04(1 \mathrm{H}, \mathrm{dd}, J=5.3,2.0 \mathrm{~Hz}), 2.05(3 \mathrm{H}, \mathrm{s}), 1.94(1 \mathrm{H}, \mathrm{s})$, 1.74-1.40 (6H, m); ${ }^{13} \mathrm{C}-\mathrm{NMR}\left(\mathrm{CDCl}_{3}, 100 \mathrm{MHz}\right) \delta 173.9,171.2,73.4,72.4,64.5,53.0,33.5$, 28.7, 22.5, 21.3; HRMS (ESI) Calc'd for [M+Na] ${ }^{+} \mathrm{C}_{10} \mathrm{H}_{18} \mathrm{O}_{6} \mathrm{Na} 257.1001$, found 257.0990.

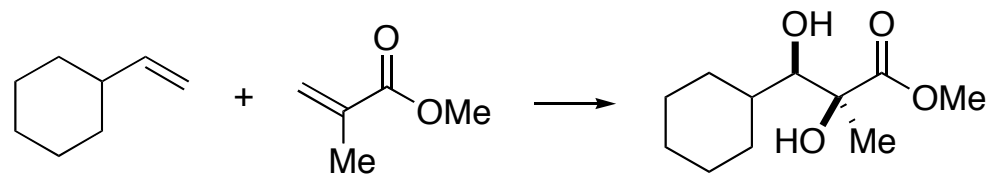

(2S, 3R)-3-Cyclohexyl-2,3-dihydroxy-2-methyl-propanoic acid methyl ester (49).

General procedure D was followed. Vinylcyclohexane (22) (65 mg, $0.59 \mathrm{mmol})$, methyl methacrylate (29) $(127 \mu \mathrm{L}, 1.18 \mathrm{mmol})$, Grubbs' catalyst 2 (25 mg, $0.029 \mathrm{mmol}), \mathrm{NaIO}_{4}(189$ $\mathrm{mg}, 0.88 \mathrm{mmol})$ and $\mathrm{CeCl}_{3} \cdot 7 \mathrm{H}_{2} \mathrm{O}(22 \mathrm{mg}, 0.059 \mathrm{mmol})$ were used in this transformation. Purification of the product by flash chromatography $\left(\mathrm{SiO}_{2}\right.$, hexanes:EtOAc, 1:1) provided diol 49 as an amber oil $(63 \mathrm{mg}, 50 \%)$. IR $\left(\mathrm{CH}_{2} \mathrm{Cl}_{2}\right.$ cast $) 3399,2921,1735 \mathrm{~cm}^{-1} ;{ }^{1} \mathbf{H}-\mathbf{N M R}\left(\mathrm{CDCl}_{3}\right.$, $400 \mathrm{MHz}) \delta 3.79(3 \mathrm{H}, \mathrm{s}), 3.58(1 \mathrm{H}, \mathrm{bs}), 3.44(1 \mathrm{H}, \mathrm{s}), 2.11(1 \mathrm{H}, \mathrm{b}), 1.86(1 \mathrm{H}, \mathrm{m}), 1.73(2 \mathrm{H}, \mathrm{m})$, 1.65-1.51 (4H, m), $1.36(3 \mathrm{H}, \mathrm{s}), 1.33-1.05(4 \mathrm{H}, \mathrm{m}) ;{ }^{13} \mathbf{C}-\mathbf{N M R}\left(\mathrm{CDCl}_{3}, 100 \mathrm{MHz}\right) \delta$ 177.3, 78.7, 77.9, 53.4, 38.9, 31.9, 27.0, 26.5, 22.9; HRMS (ESI) Calc'd for [M+Na] ${ }^{+} \mathrm{C}_{11} \mathrm{H}_{20} \mathrm{O}_{4} \mathrm{Na} 239.1259$, 
found 239.1264.<smiles>C=CCOC(C)=O</smiles>

$(2 S, 3 R)$-7-Acetoxy-2,3-dihydroxy-2-methylheptanoic acid acid methyl ester (50).

General procedure D was followed. 1-Hexen-6-yl acetate (25) $(67 \mathrm{mg}, 0.47 \mathrm{mmol})$, methyl methacrylate (29) $(101 \mu \mathrm{L}, 0.94 \mathrm{mmol})$, Grubbs' catalyst $2(20 \mathrm{mg}, 0.024 \mathrm{mmol}), \mathrm{NaIO}_{4}(151$ $\mathrm{mg}, 0.71 \mathrm{mmol})$ and $\mathrm{CeCl}_{3} \cdot 7 \mathrm{H}_{2} \mathrm{O}(18 \mathrm{mg}, 0.047 \mathrm{mmol})$ were used in this transformation. Purification of the product by flash chromatography $\left(\mathrm{SiO}_{2}\right.$, hexanes:EtOAc, 1:1) provided diol 50 as an amber oil $(89 \mathrm{mg}, 76 \%)$. IR $\left(\mathrm{CH}_{2} \mathrm{Cl}_{2}\right.$ cast $) 3432,2955,2329,1735,1256 \mathrm{~cm}^{-1}$; ${ }^{1} \mathbf{H}-\mathbf{N M R}\left(\mathrm{CDCl}_{3}, 400 \mathrm{MHz}\right) \delta 4.05(3 \mathrm{H}, \mathrm{m}), 3.50(3 \mathrm{H}, \mathrm{s}), 3.40(2 \mathrm{H}, \mathrm{m}), 2.03(4 \mathrm{H}, \mathrm{m}), 1.92(1 \mathrm{H}$, m), $1.32(3 \mathrm{H}, \mathrm{s}), 1.72-1.60(2 \mathrm{H}, \mathrm{m}), 1.46-1.32(2 \mathrm{H}, \mathrm{m}) ;{ }^{13} \mathbf{C}-\mathbf{N M R}\left(\mathrm{CDCl}_{3}, 100 \mathrm{MHz}\right) \delta 176.6$, 171.3, 77.7, 75.5, 64.6, 53.2, 30.0 28.7, 22.6, 22.0 21.3; HRMS (ESI) Calc'd for $[\mathrm{M}+\mathrm{Na}]^{+}$ $\mathrm{C}_{11} \mathrm{H}_{20} \mathrm{O}_{6} \mathrm{Na} 271.1158$, found 271.1146.<smiles>C=C[C+]=CC(=O)OCC</smiles>

(2S, 3R)-2,3-Dihydroxy-3-phenyl-propionic acid methyl ester (51). ${ }^{8}$

General procedure D was followed. Styrene (27) $(51 \mathrm{mg}, 0.49 \mathrm{mmol})$, methylacrylate (23) ( $88 \mu \mathrm{L}, 0.98 \mathrm{mmol})$, Grubbs' catalyst $2(21 \mathrm{mg}, 0.025 \mathrm{mmol}), \mathrm{NaIO}_{4}(157 \mathrm{mg}, 0.74 \mathrm{mmol})$ and $\mathrm{CeCl}_{3} \cdot 7 \mathrm{H}_{2} \mathrm{O}(18 \mathrm{mg}, 0.049 \mathrm{mmol})$ were used in this transformation. Purification of the product by flash chromatography $\left(\mathrm{SiO}_{2}\right.$, hexanes:EtOAc, 1:1) provided diol $\mathbf{5 1}$ as an amber oil $(52 \mathrm{mg}$, 56\%). IR $\left(\mathrm{CH}_{2} \mathrm{Cl}_{2}\right.$ cast $) 3395,3032,2954,2872,1739,1439,1276,1120 \mathrm{~cm}^{-1} ;{ }^{1} \mathbf{H}-\mathbf{N M R}$ $\left(\mathrm{CDCl}_{3}, 400 \mathrm{MHz}\right) \delta$ 7.43-7.32 (5H, m), $5.04(1 \mathrm{H}, \mathrm{dd}, J=7.3,2.9 \mathrm{~Hz}), 4.39(1 \mathrm{H}, \mathrm{dd}, J=6.1,2.9$ $\mathrm{Hz}), 3.83(3 \mathrm{H}, \mathrm{s}), 3.06(1 \mathrm{H}, \mathrm{d}, J=6.1 \mathrm{~Hz}), 2.66(1 \mathrm{H}, \mathrm{d}, J=7.3 \mathrm{~Hz}) ;{ }^{13} \mathbf{C}-\mathbf{N M R}\left(\mathrm{CDCl}_{3}, 100\right.$ $\mathrm{MHz}) \delta 173.1,139.9,128.5,128.1,126.3,75.0,74.6,53.1$; HRMS (ESI) Calc'd for $[\mathrm{M}+\mathrm{Na}]^{+}$ $\mathrm{C}_{10} \mathrm{H}_{22} \mathrm{O}_{4} \mathrm{Na} 219.0633$, found 219.0641.<smiles>C=CC(=O)OC</smiles>

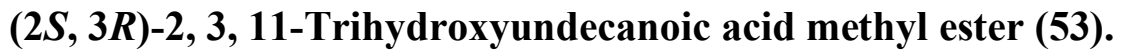

General procedure D was followed. 9-Decen-1-ol (52) $(51 \mathrm{mg}, 0.33 \mathrm{mmol})$, methylacrylate (23) $(59 \mu \mathrm{L}, 0.65 \mathrm{mmol})$, Grubbs' catalyst 2 (14 mg, $0.016 \mathrm{mmol}), \mathrm{NaIO}_{4}(105 \mathrm{mg}, 0.49 \mathrm{mmol})$ and $\mathrm{CeCl}_{3} \cdot 7 \mathrm{H}_{2} \mathrm{O}(12 \mathrm{mg}, 0.033 \mathrm{mmol})$ were used in this transformation. Purification of the product by flash chromatography $\left(\mathrm{SiO}_{2}\right.$, hexanes:EtOAc, 1:2) provided diol $\mathbf{5 3}$ as an amber oil (44 mg, 54\%). IR $\left(\mathrm{CH}_{2} \mathrm{Cl}_{2}\right.$ cast) 3411, 2951, 2859, $1739 \mathrm{~cm}^{-1} ;{ }^{1} \mathbf{H}-\mathbf{N M R}\left(\mathrm{CDCl}_{3}, 400 \mathrm{MHz}\right) \delta$ $4.08(1 \mathrm{H}, \mathrm{m}), 3.86(1 \mathrm{H}, \mathrm{m}), 3.81(3 \mathrm{H}, \mathrm{s}), 3.61(2 \mathrm{H}, \mathrm{t}, J=6.8 \mathrm{~Hz}), 3.03(1 \mathrm{H}, \mathrm{d}, J=5.6 \mathrm{~Hz}), 1.90$ $(1 \mathrm{H}, \mathrm{m}), 1.62-1.50(5 \mathrm{H}, \mathrm{m}), 1.47-1.41(1 \mathrm{H}, \mathrm{m}), 1.38-1.24(8 \mathrm{H}, \mathrm{m}) ;{ }^{13} \mathbf{C}-\mathbf{N M R}\left(\mathrm{CDCl}_{3}, 100 \mathrm{MHz}\right)$ $\delta$ 174.0, 73.2, 72.6, 63.3, 53.1, 34.0, 33.0, 29.7, 29.6, 26.0; HRMS (ESI) Calc'd for $[\mathrm{M}+\mathrm{Na}]^{+}$ $\mathrm{C}_{12} \mathrm{H}_{24} \mathrm{O}_{5} \mathrm{Na} 271.1521$, found 271.1508. 


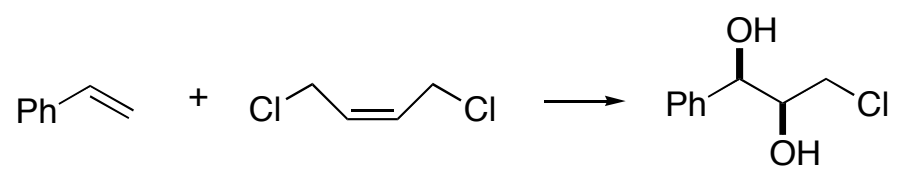

\section{$(1 S, 2 S)$-Chloro-1-phenyl-propane-1,2-diol (54). ${ }^{4}$}

General procedure D was followed. Styrene (27) $(70 \mathrm{mg}, 0.67 \mathrm{mmol})$, cis-1,4-dichloro-2butene (32) $(141 \mu \mathrm{L}, 1.34 \mathrm{mmol})$, Grubbs' catalyst $2(28 \mathrm{mg}, 0.037 \mathrm{mmol}), \mathrm{NaIO}_{4}(431 \mathrm{mg}$, $2.016 \mathrm{mmol})$ and $\mathrm{CeCl}_{3} \cdot 7 \mathrm{H}_{2} \mathrm{O}(50 \mathrm{mg}, 0.13 \mathrm{mmol})$ were used in this transformation. Purification of the product by flash chromatography $\left(\mathrm{SiO}_{2}\right.$, hexanes:EtOAc, $\left.1: 1\right)$ provided diol $\mathbf{5 4}$ as an amber oil (53 mg, 42\%). IR ( $\mathrm{CH}_{2} \mathrm{Cl}_{2}$ cast) 3383, 2833, 1633, 1433, $1058 \mathrm{~cm}^{-1}$; ${ }^{1} \mathbf{H}-\mathbf{N M R}$ $\left(\mathrm{CDCl}_{3}, 400 \mathrm{MHz}\right) \delta 7.35(5 \mathrm{H}, \mathrm{m}), 4.70(1 \mathrm{H}, \mathrm{d}, J=6.8 \mathrm{~Hz}), 3.89(2 \mathrm{H}, \mathrm{m}), 3.61(1 \mathrm{H}, \mathrm{m}), 3.53$ $(1 \mathrm{H}, \mathrm{dd}, J=11.6,3.8 \mathrm{~Hz}), 3.35(1 \mathrm{H}, \mathrm{dd}, J=11.6,6.8 \mathrm{~Hz}), 3.18(1 \mathrm{H}, \mathrm{bs}), 2.98(1 \mathrm{H}, \mathrm{bs})$; ${ }^{13} \mathbf{C}-\mathbf{N M R}\left(\mathrm{CDCl}_{3}, 100 \mathrm{MHz}\right) \delta 139.8,125.8,128.6,126.7,75.6,71.3,46.3$; HRMS (EI) Calc'd for $[\mathrm{M}+] \mathrm{C}_{9} \mathrm{H}_{11} \mathrm{ClO}_{2}$ 186.0448, found 186.0455 . 


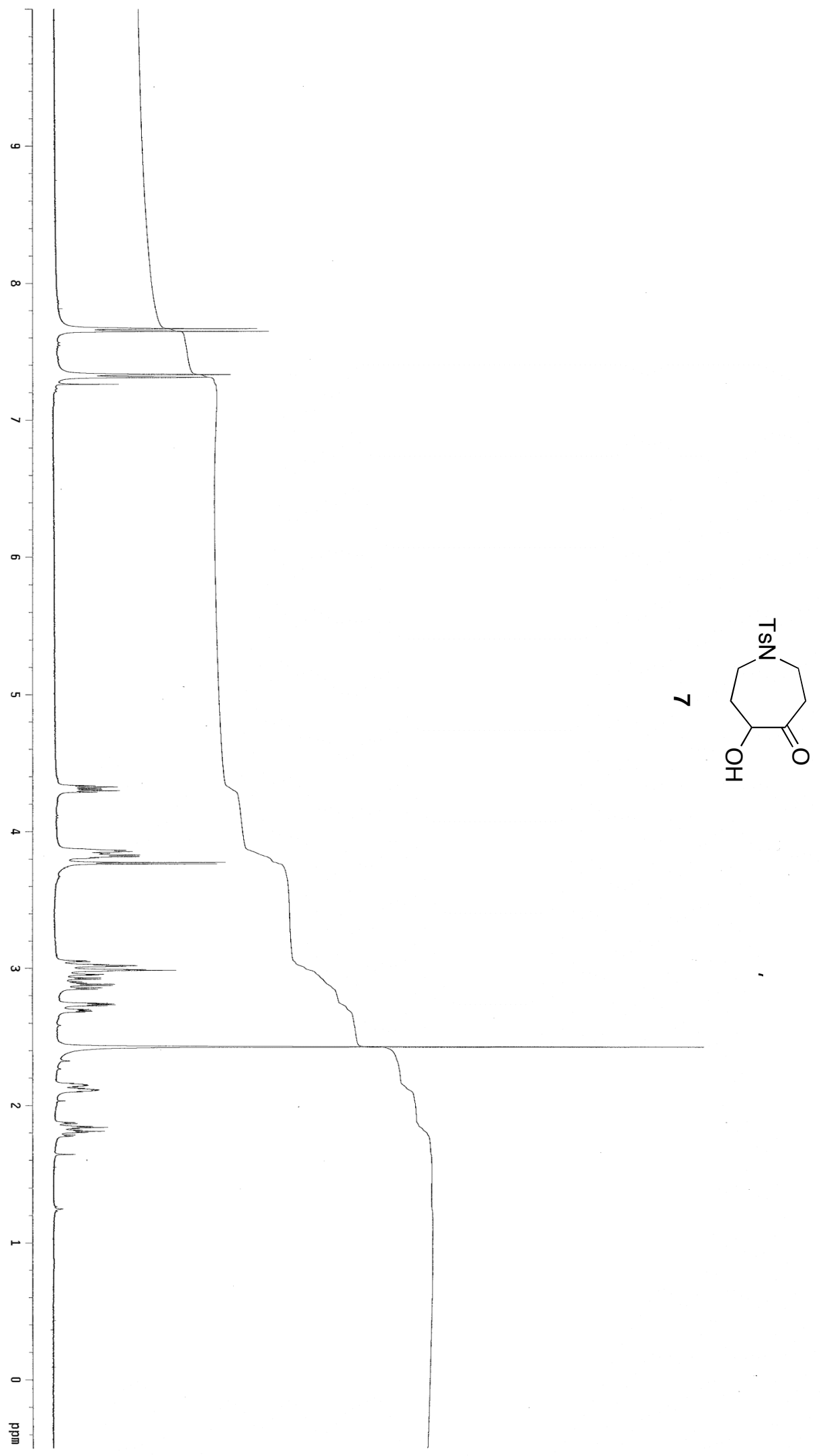




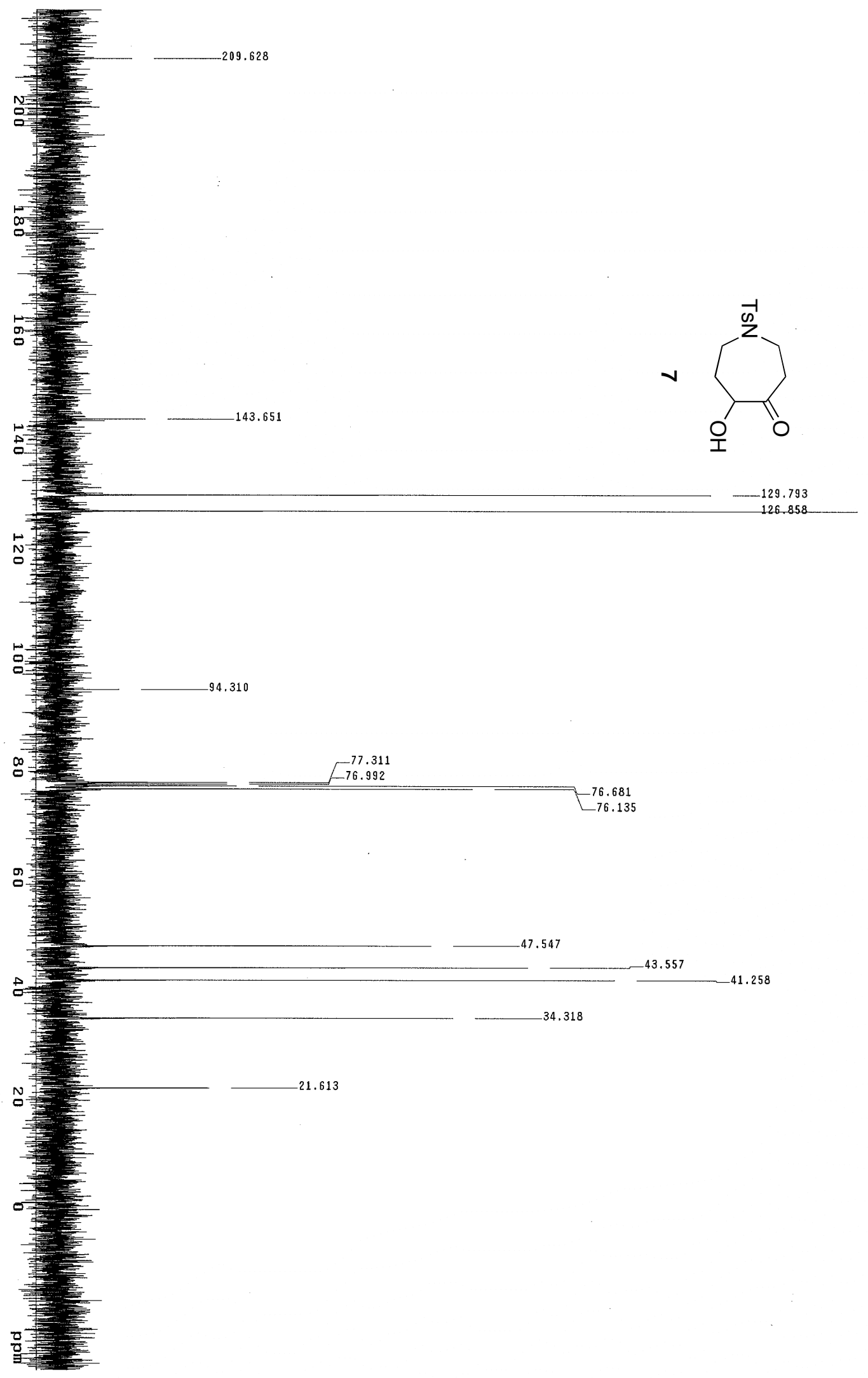




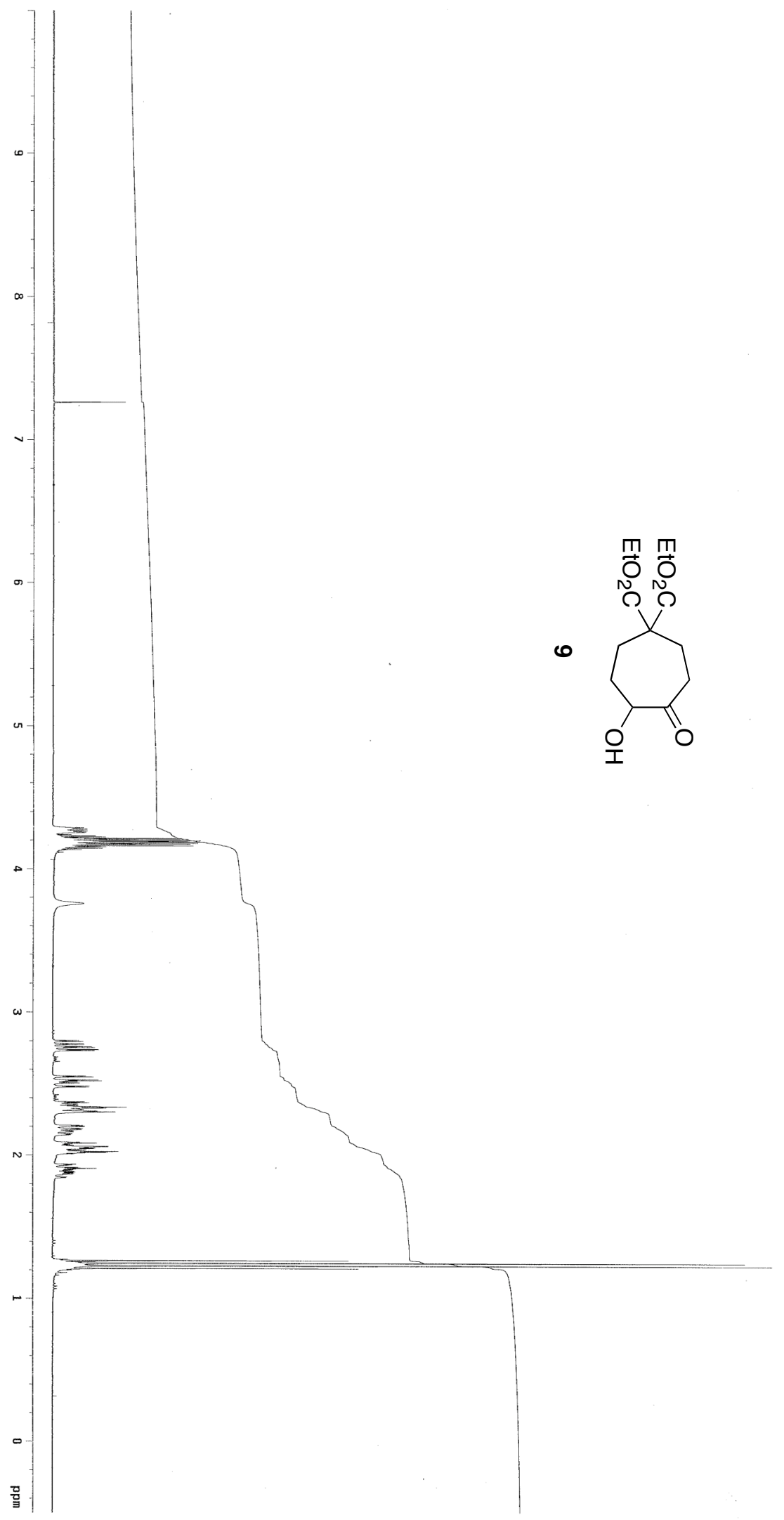




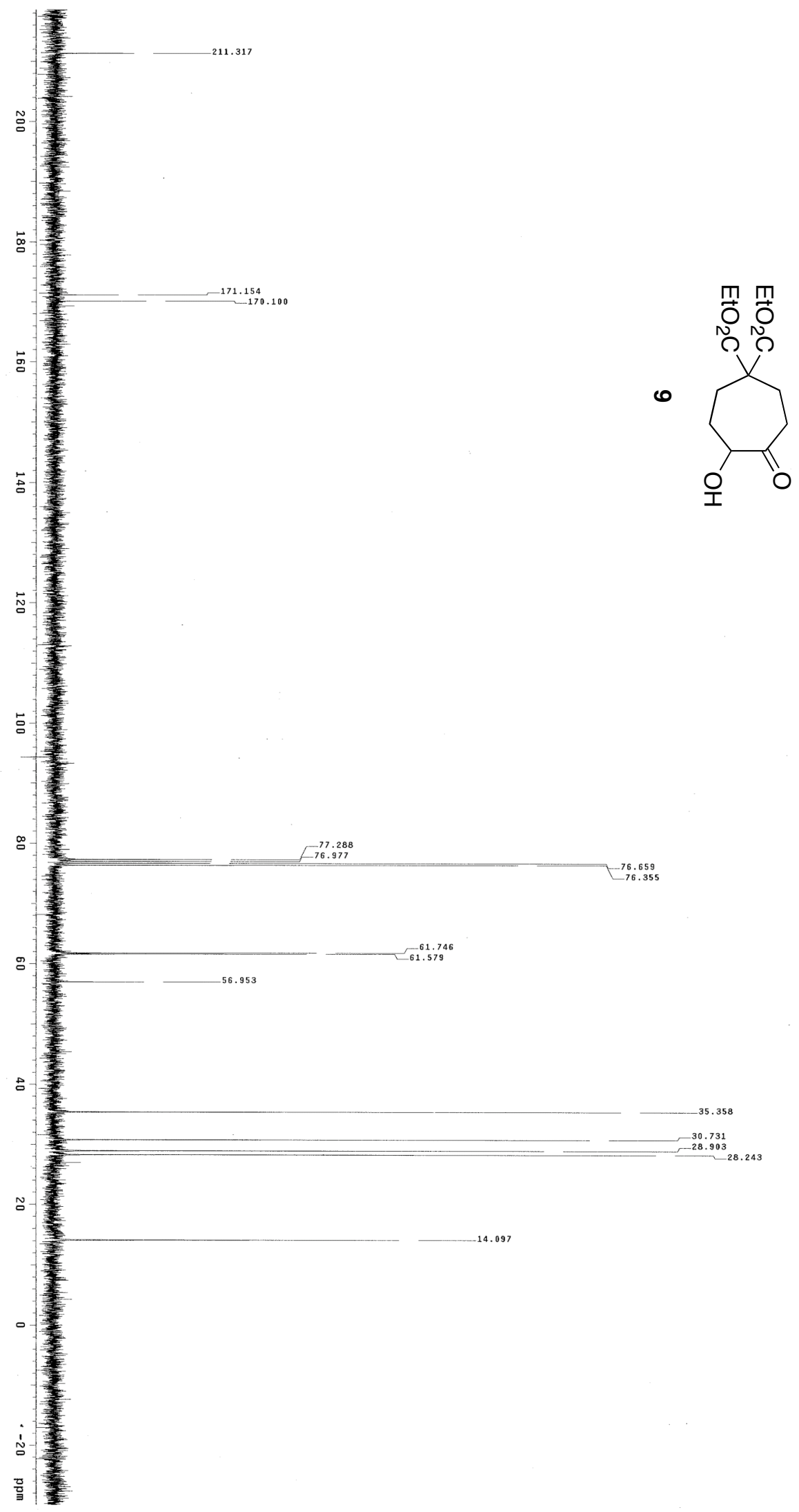




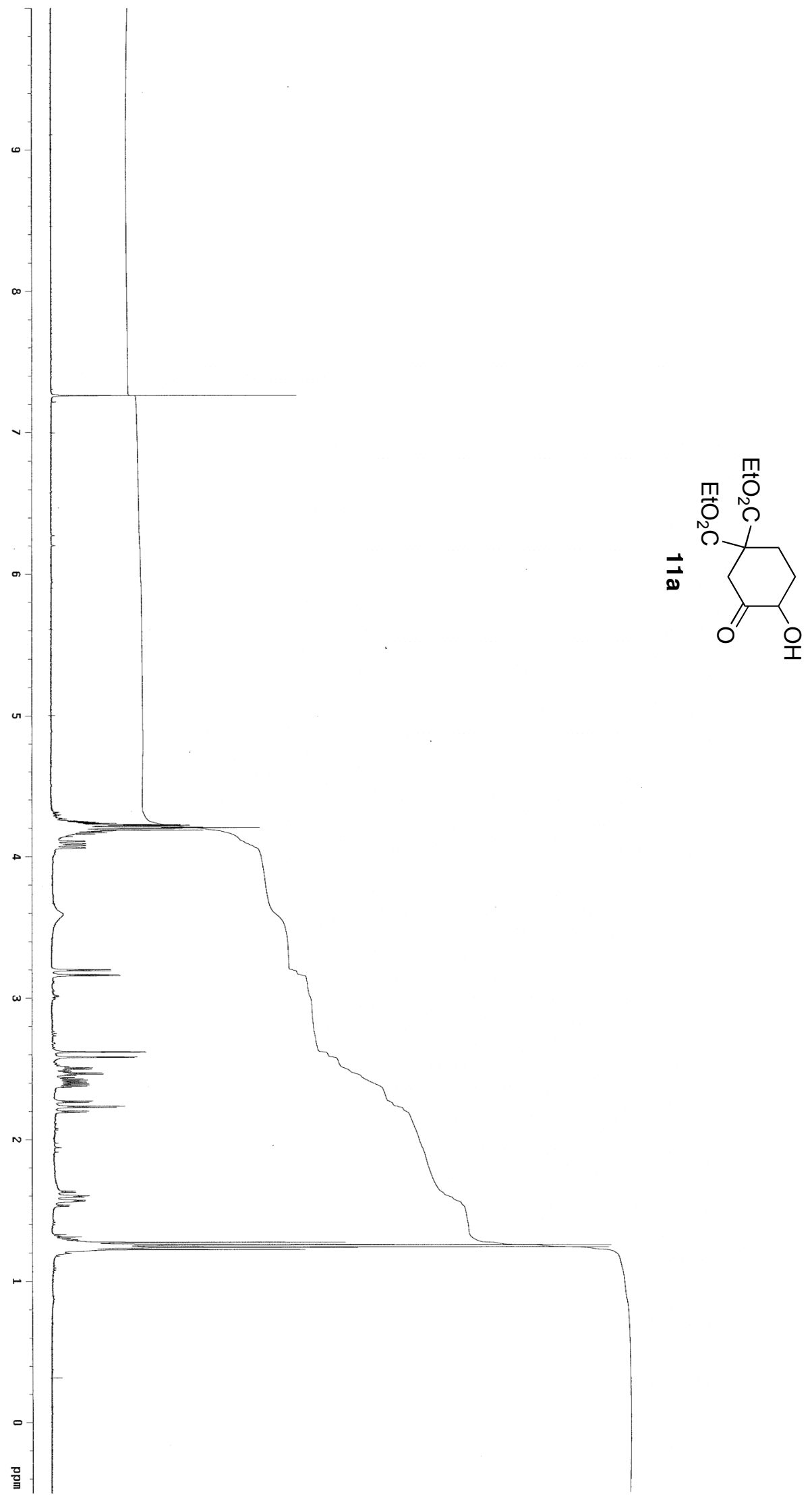




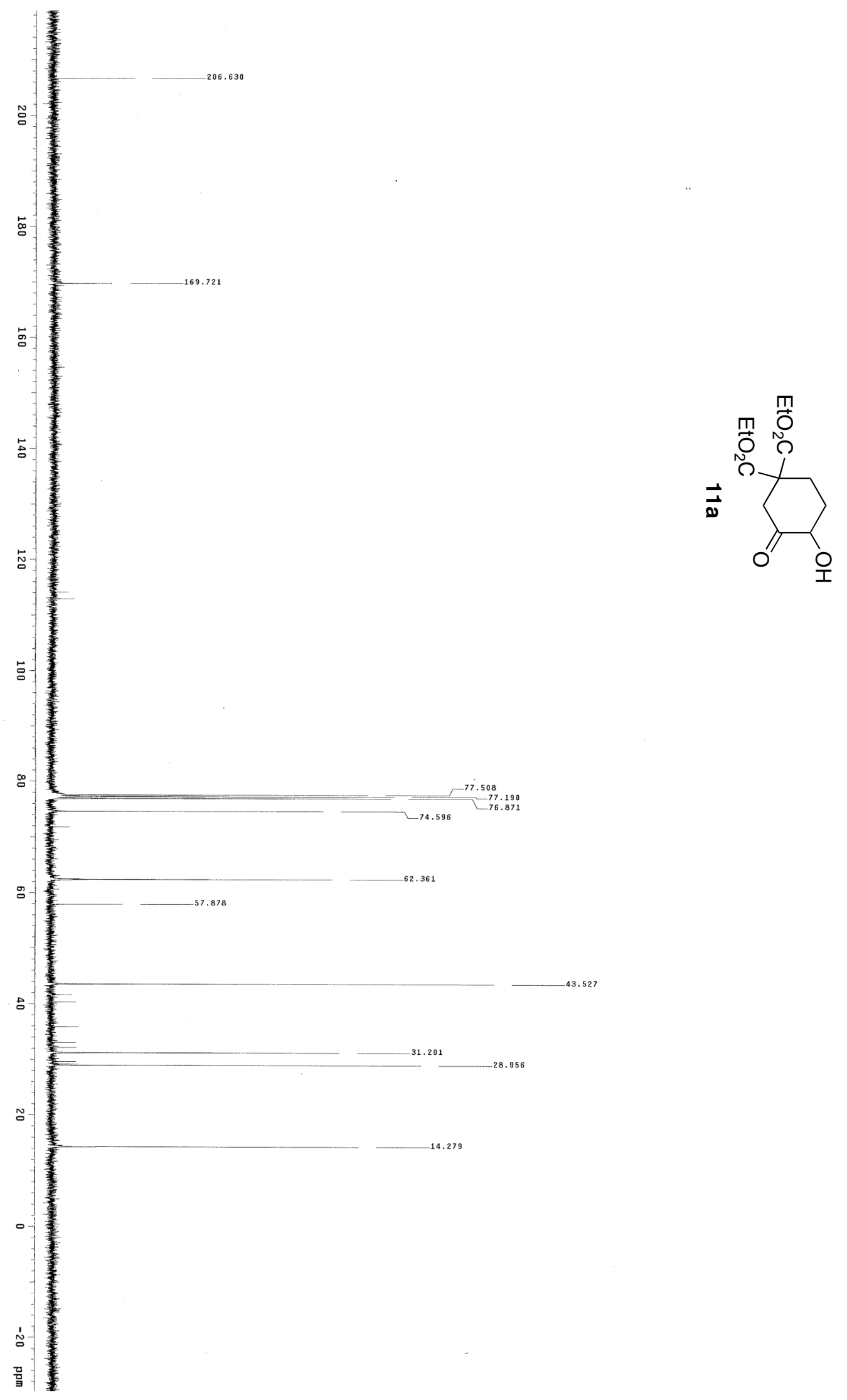




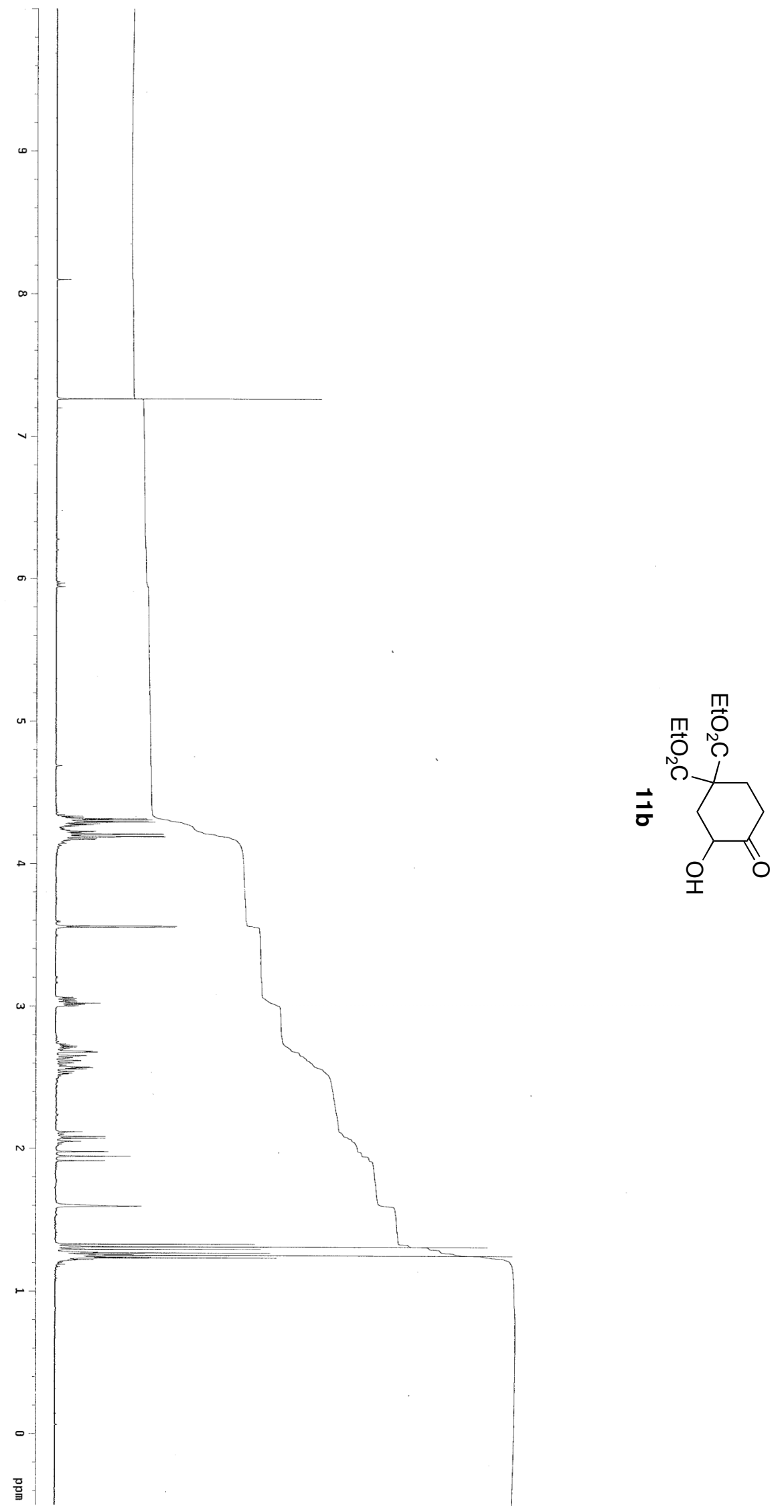



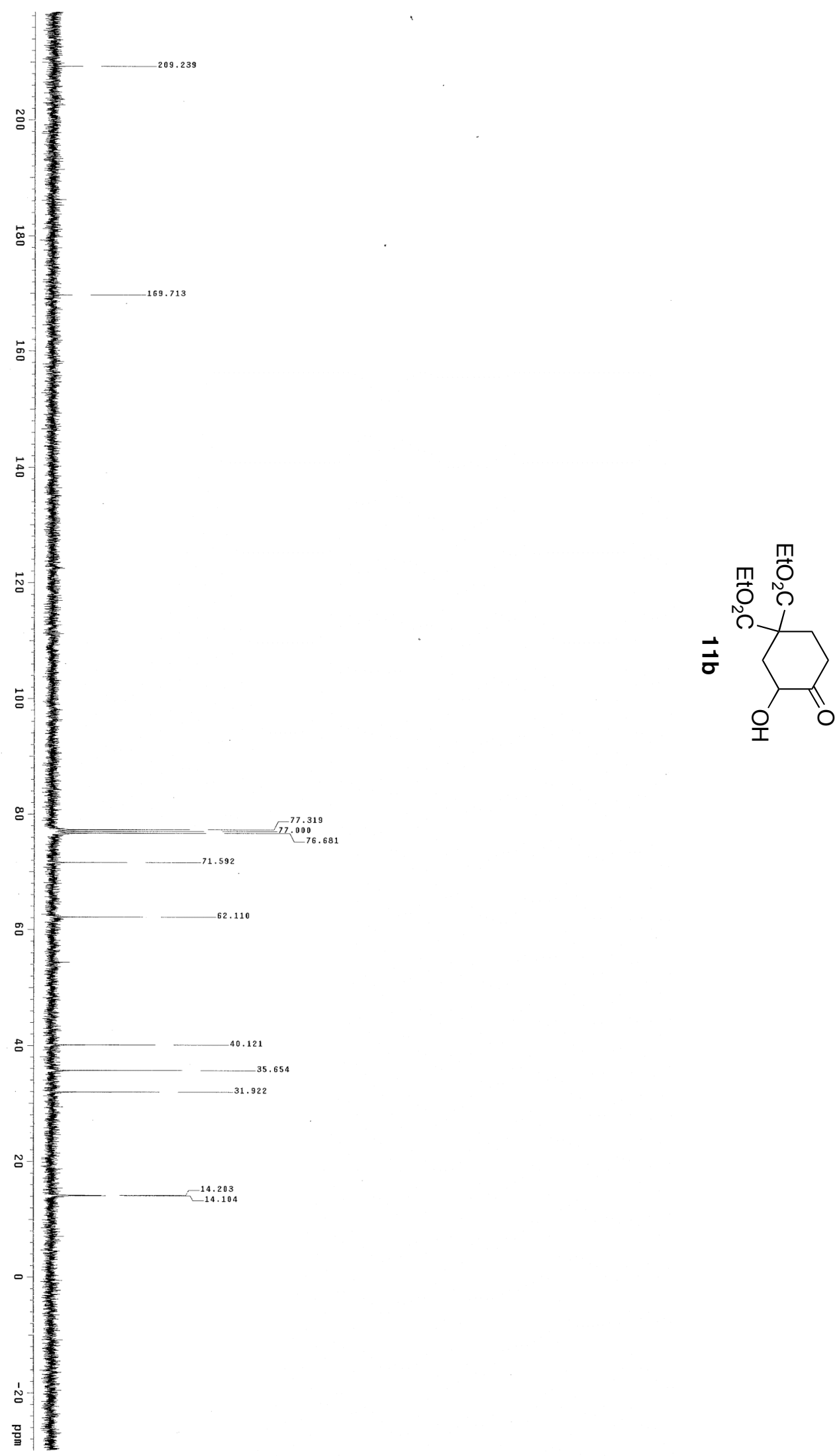


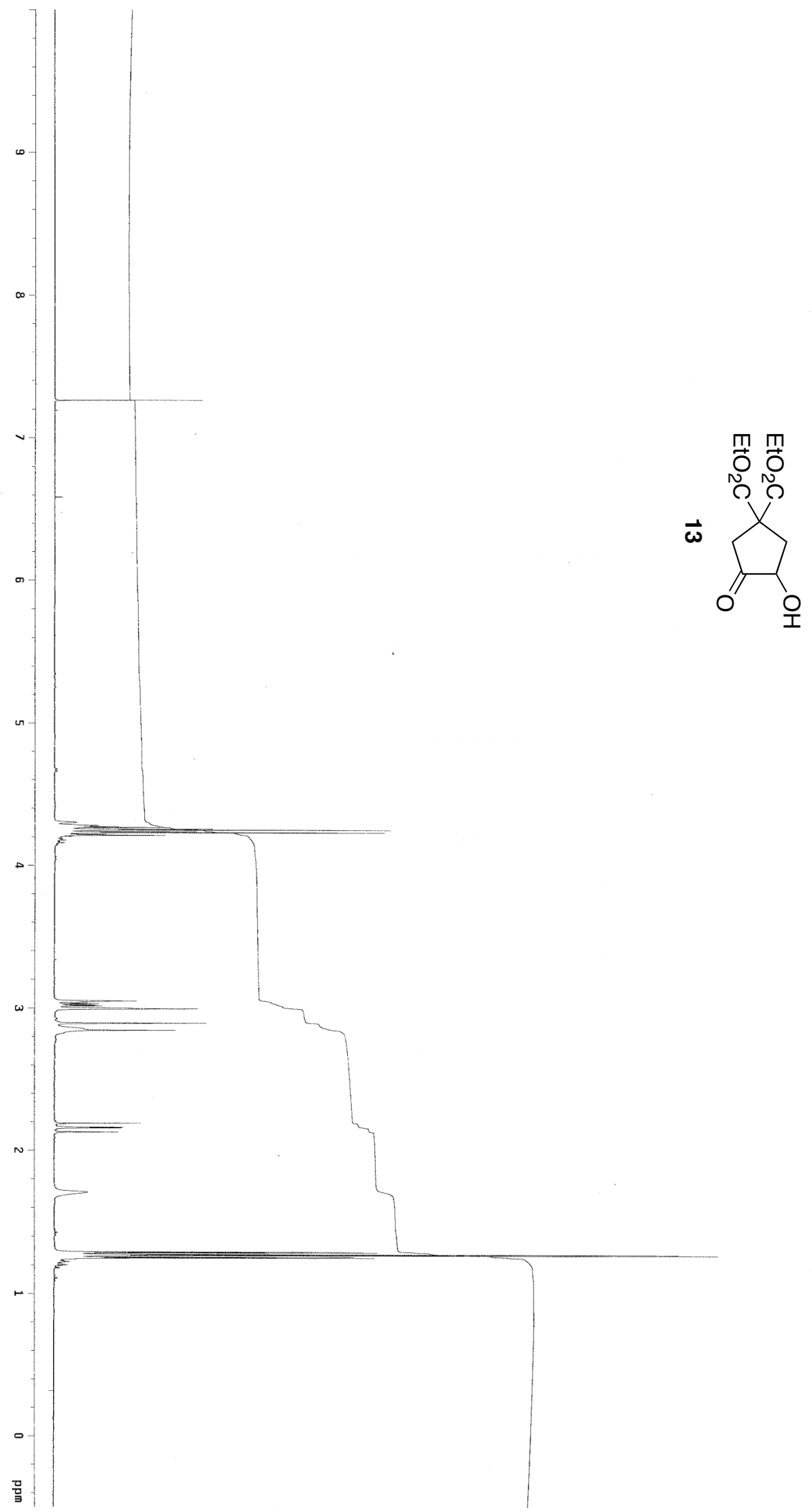



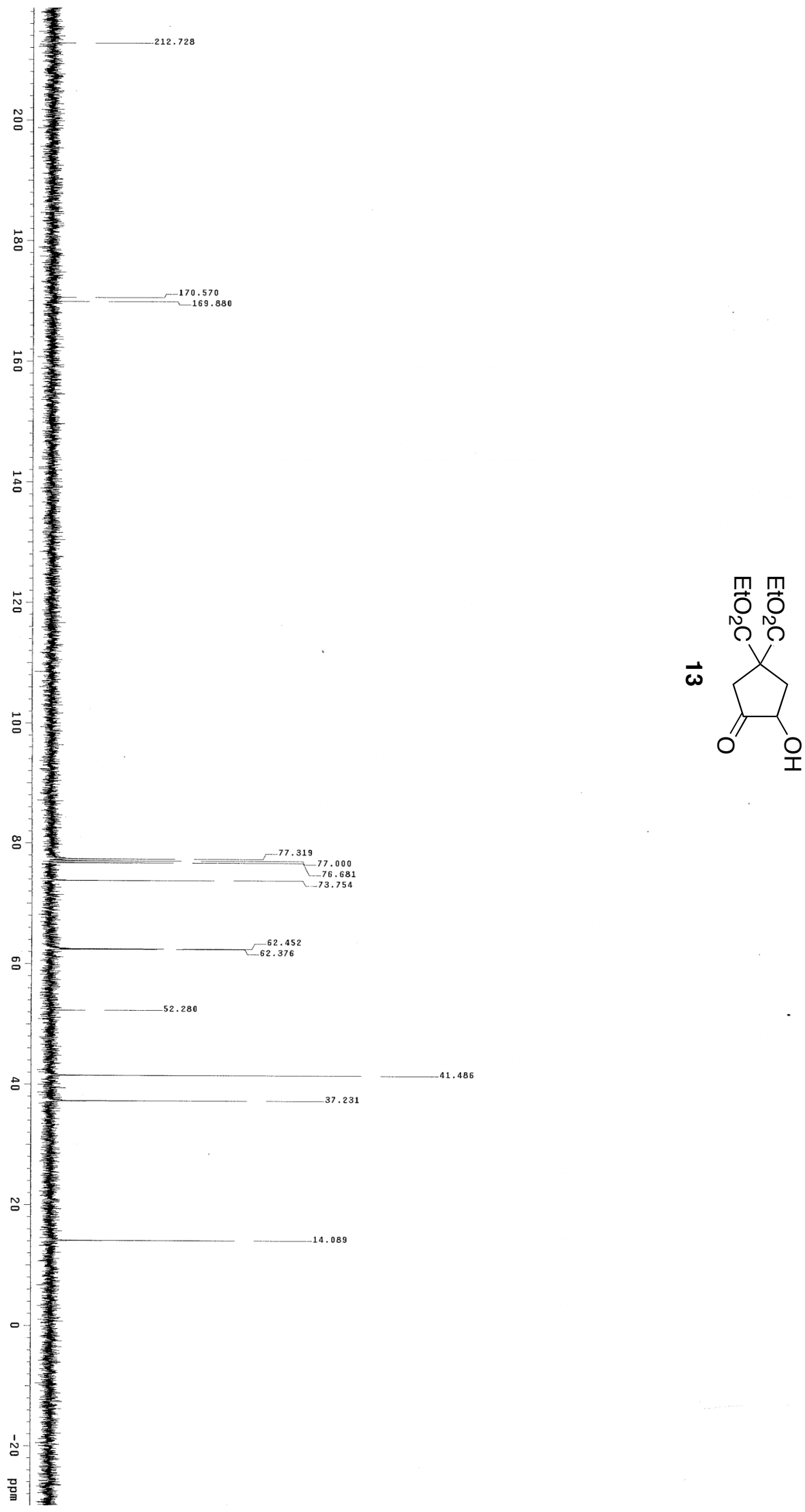


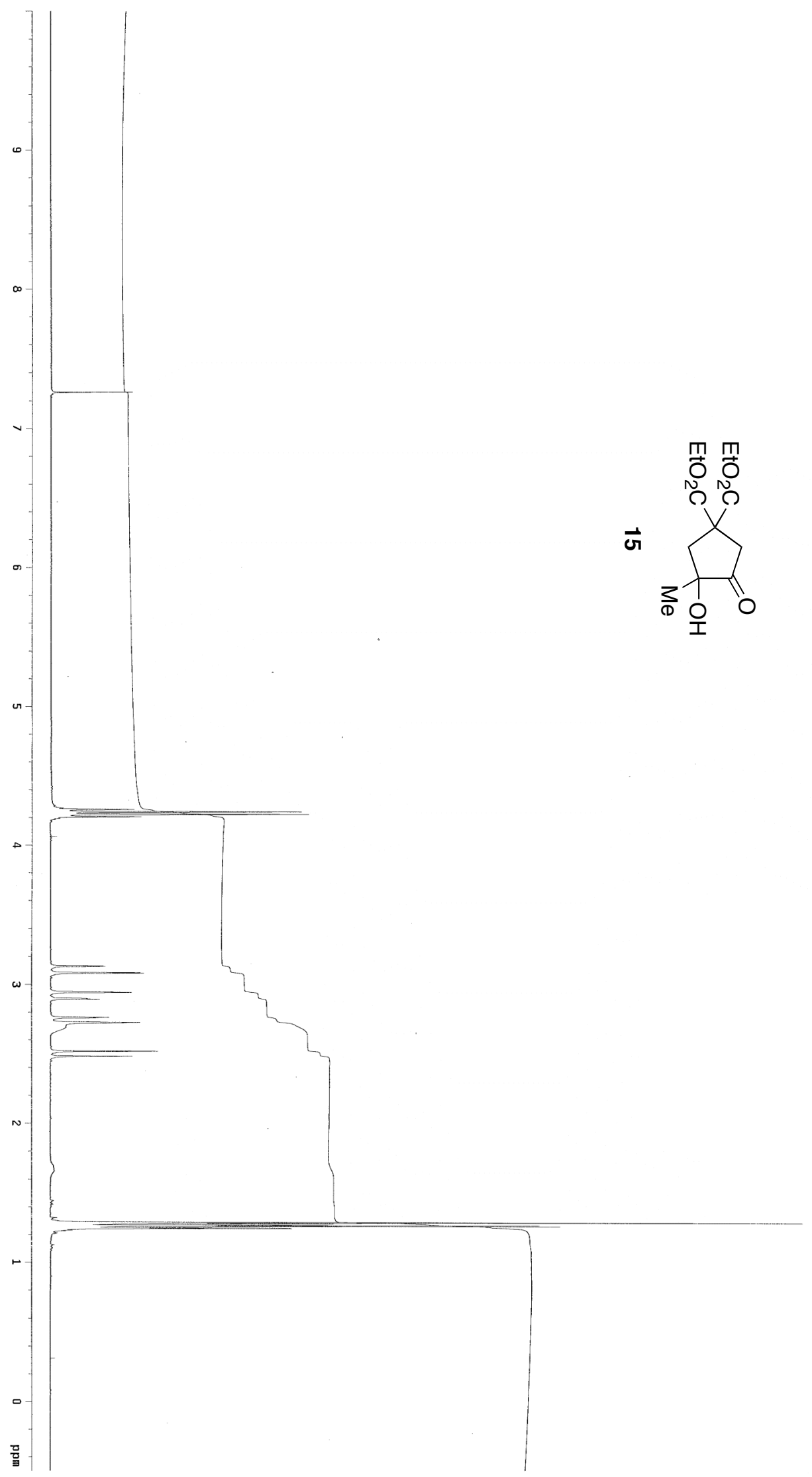



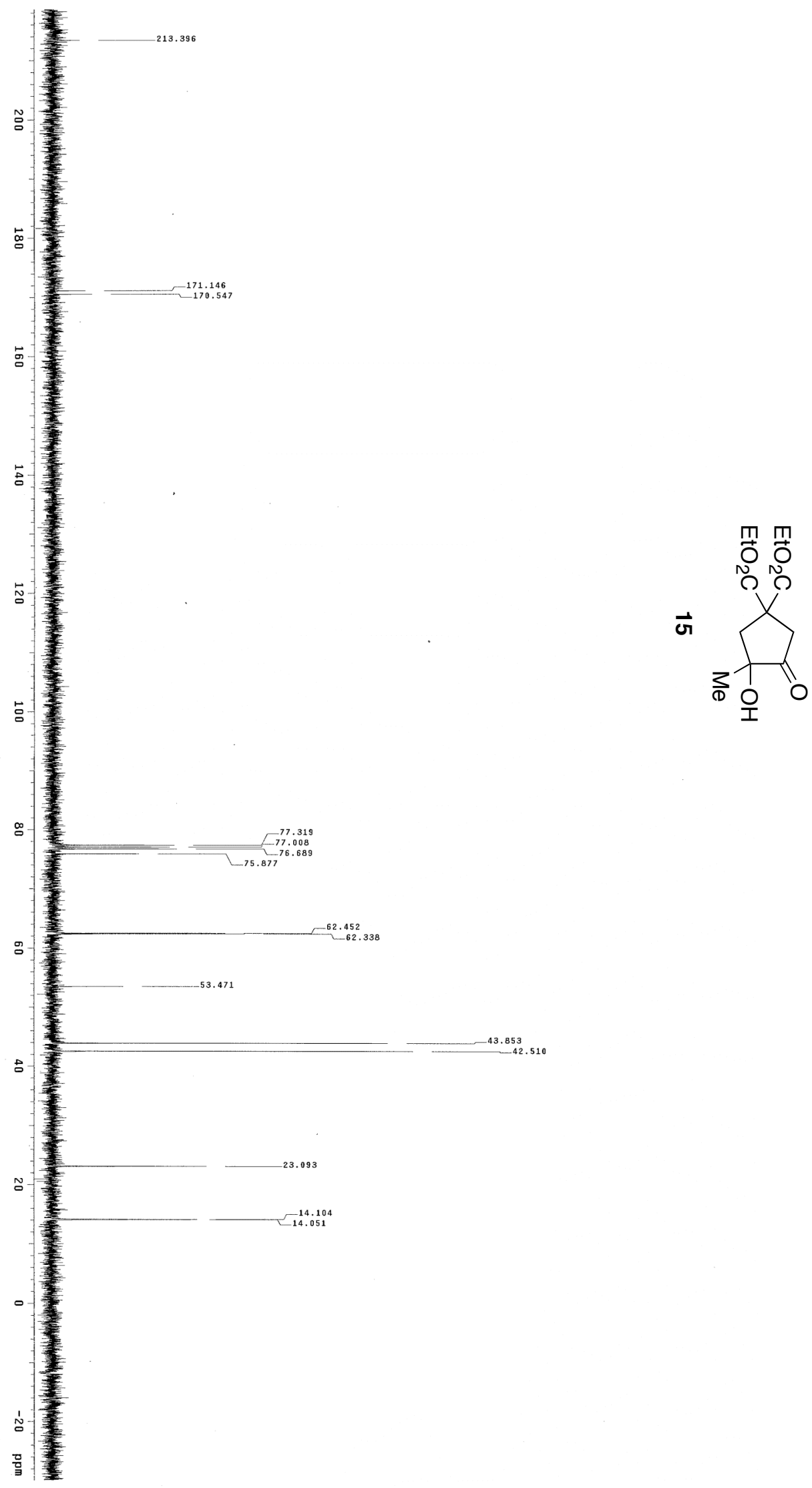


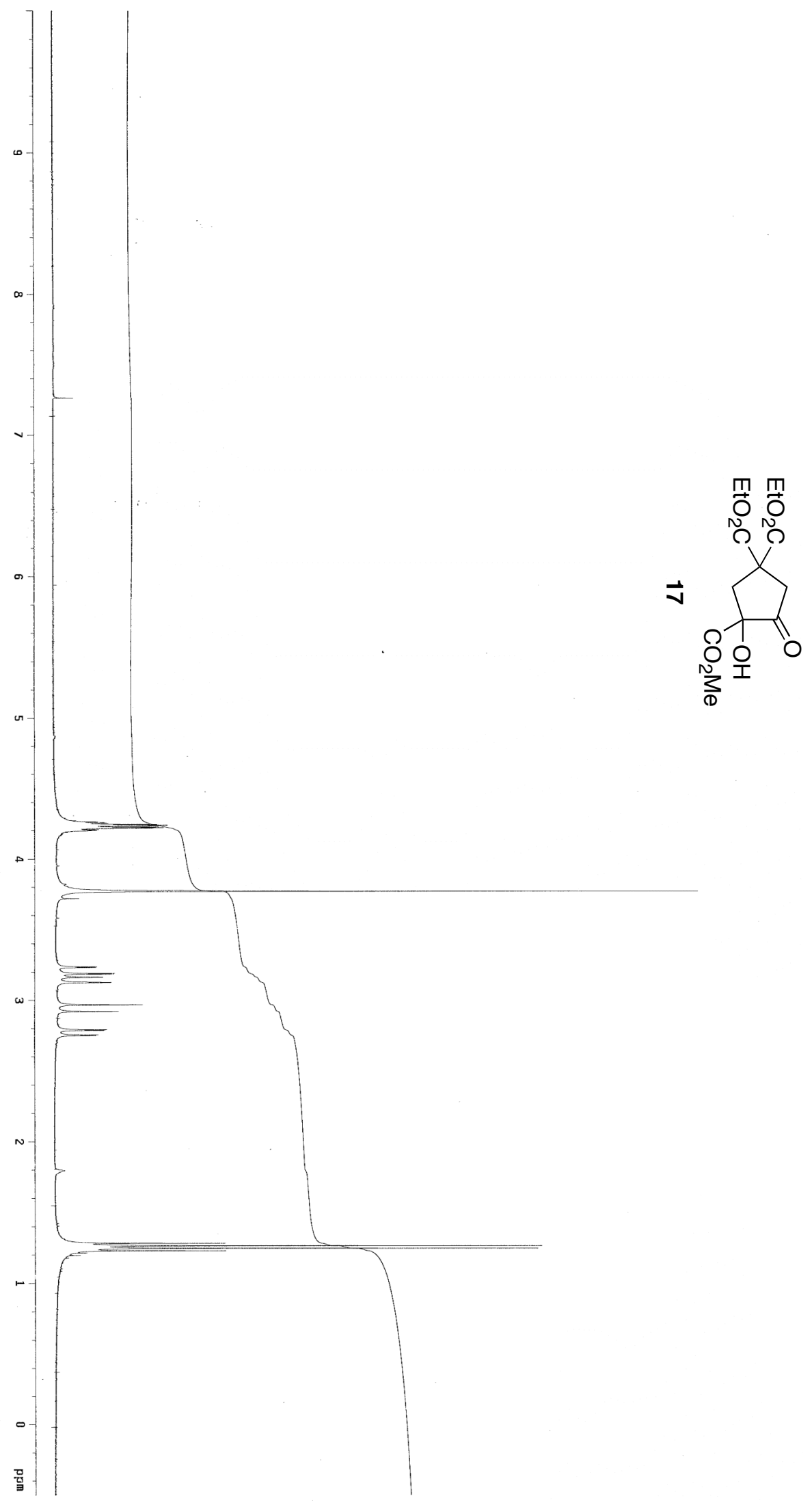




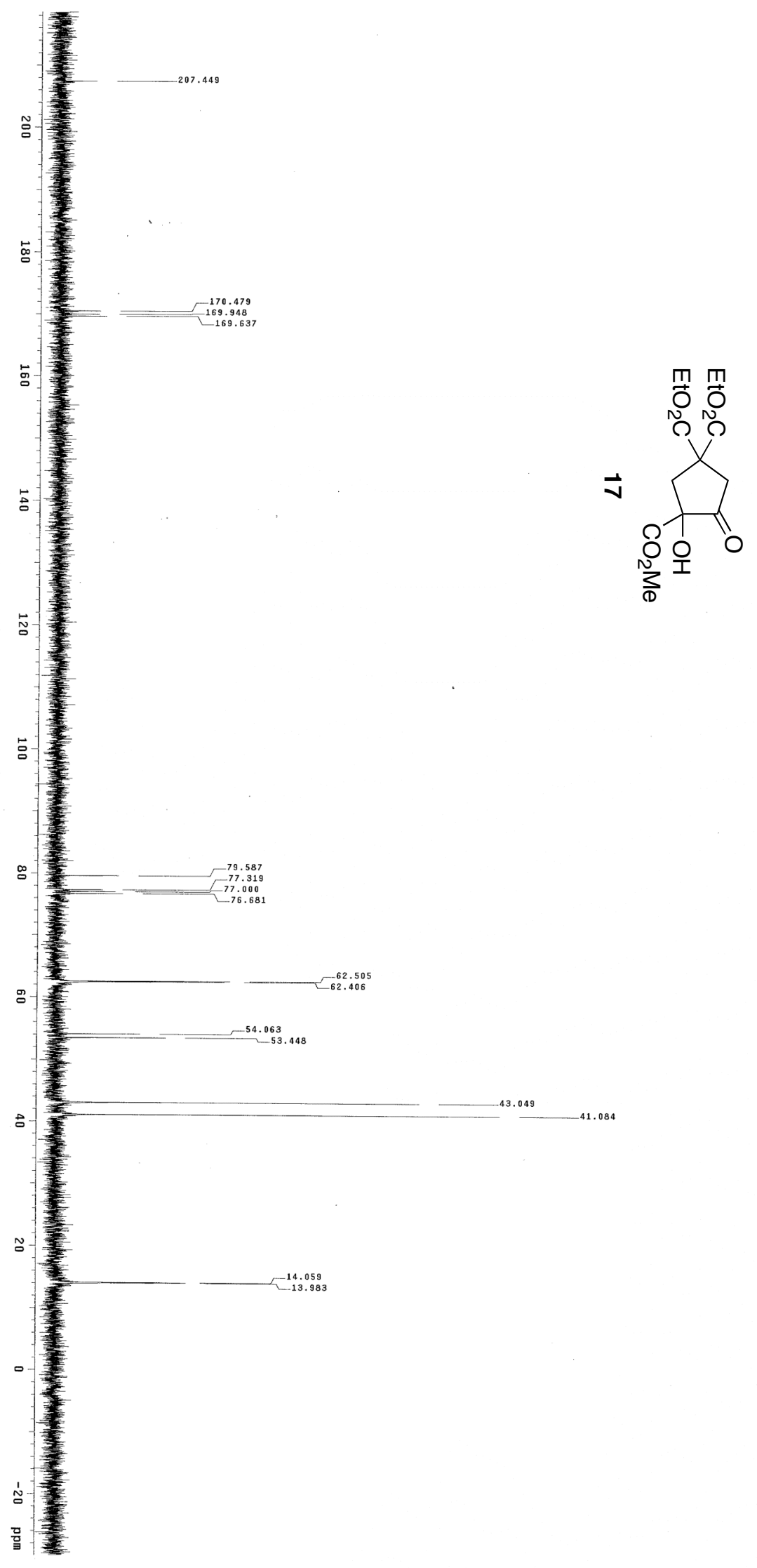



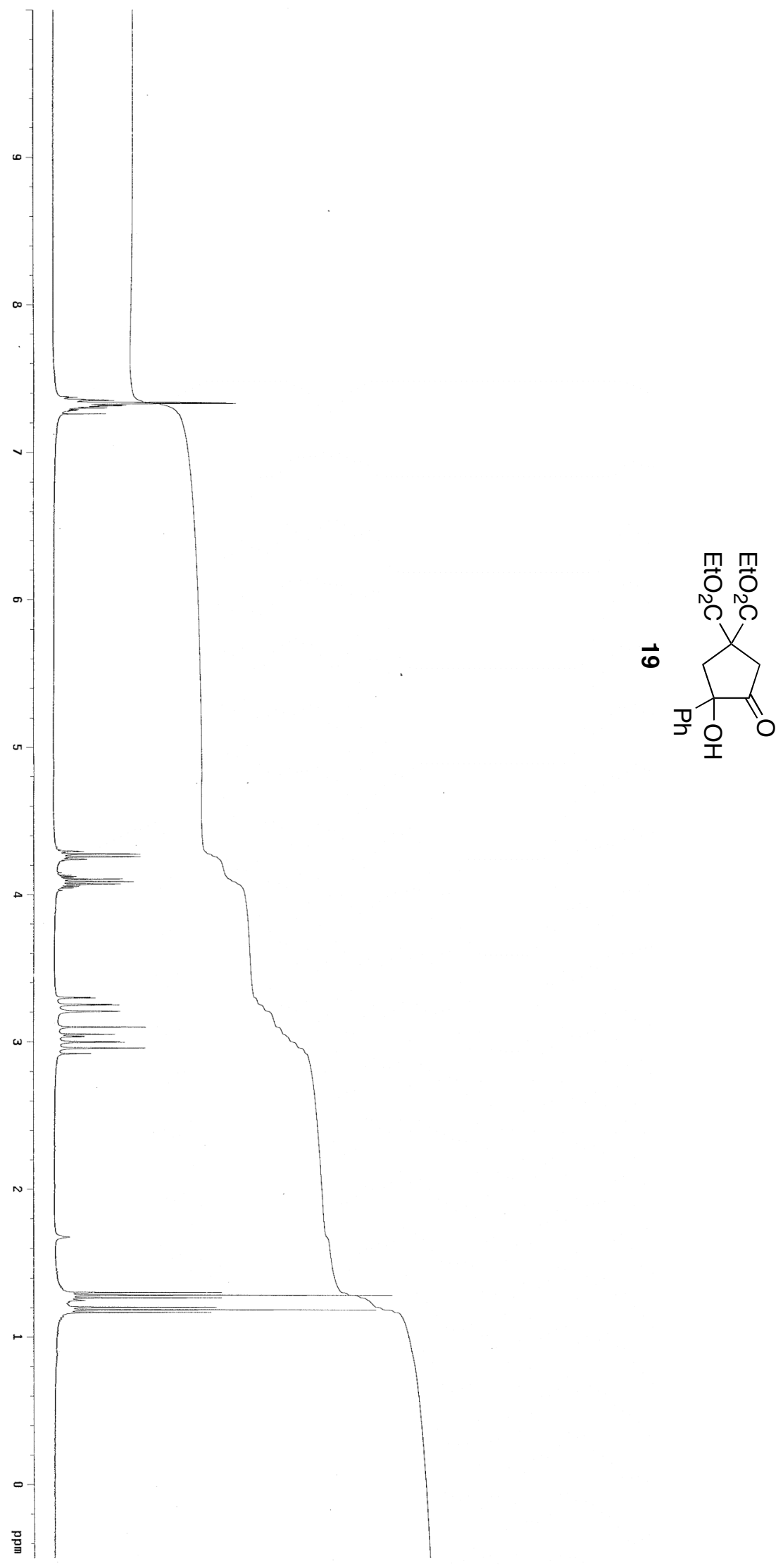

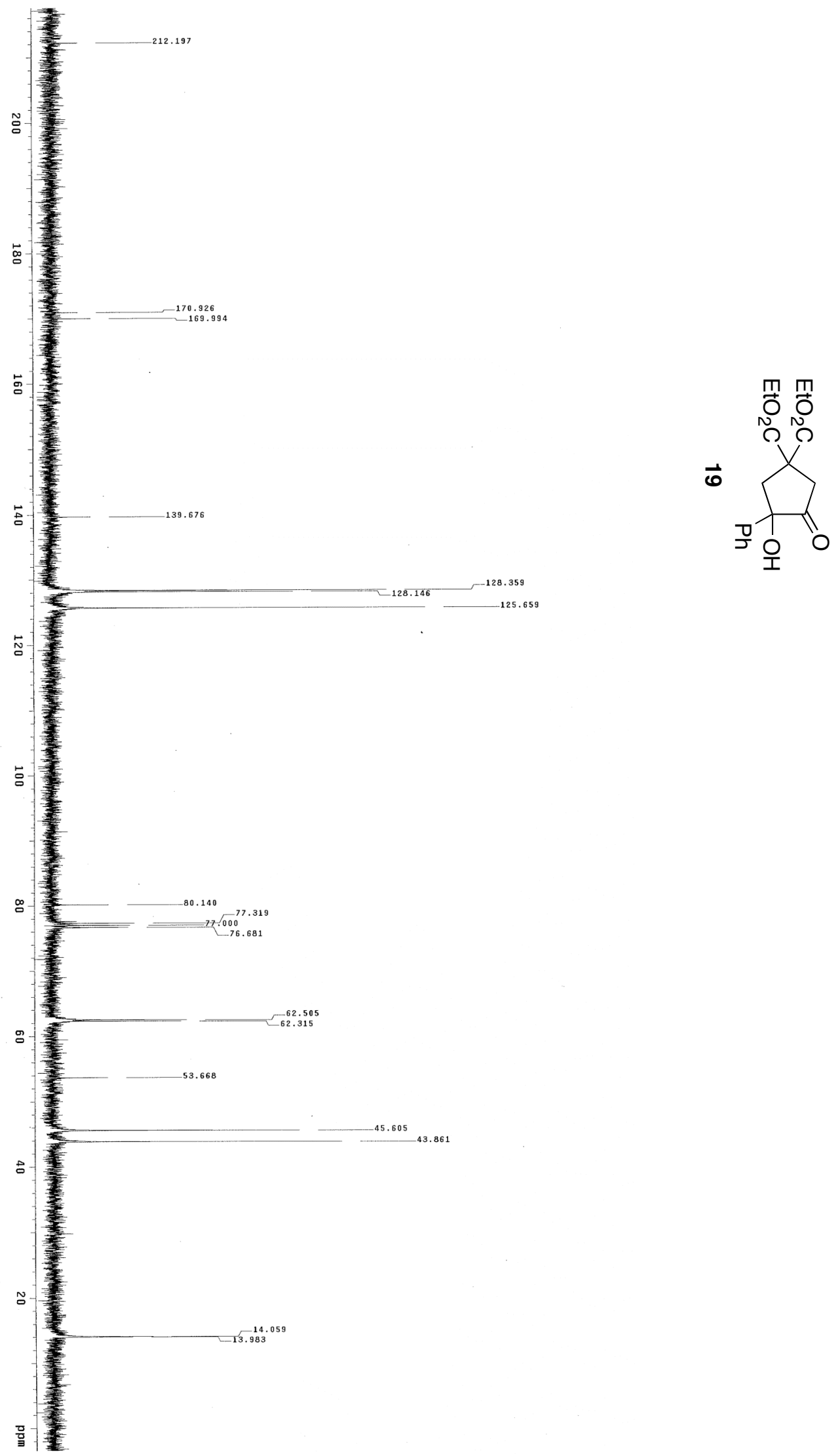


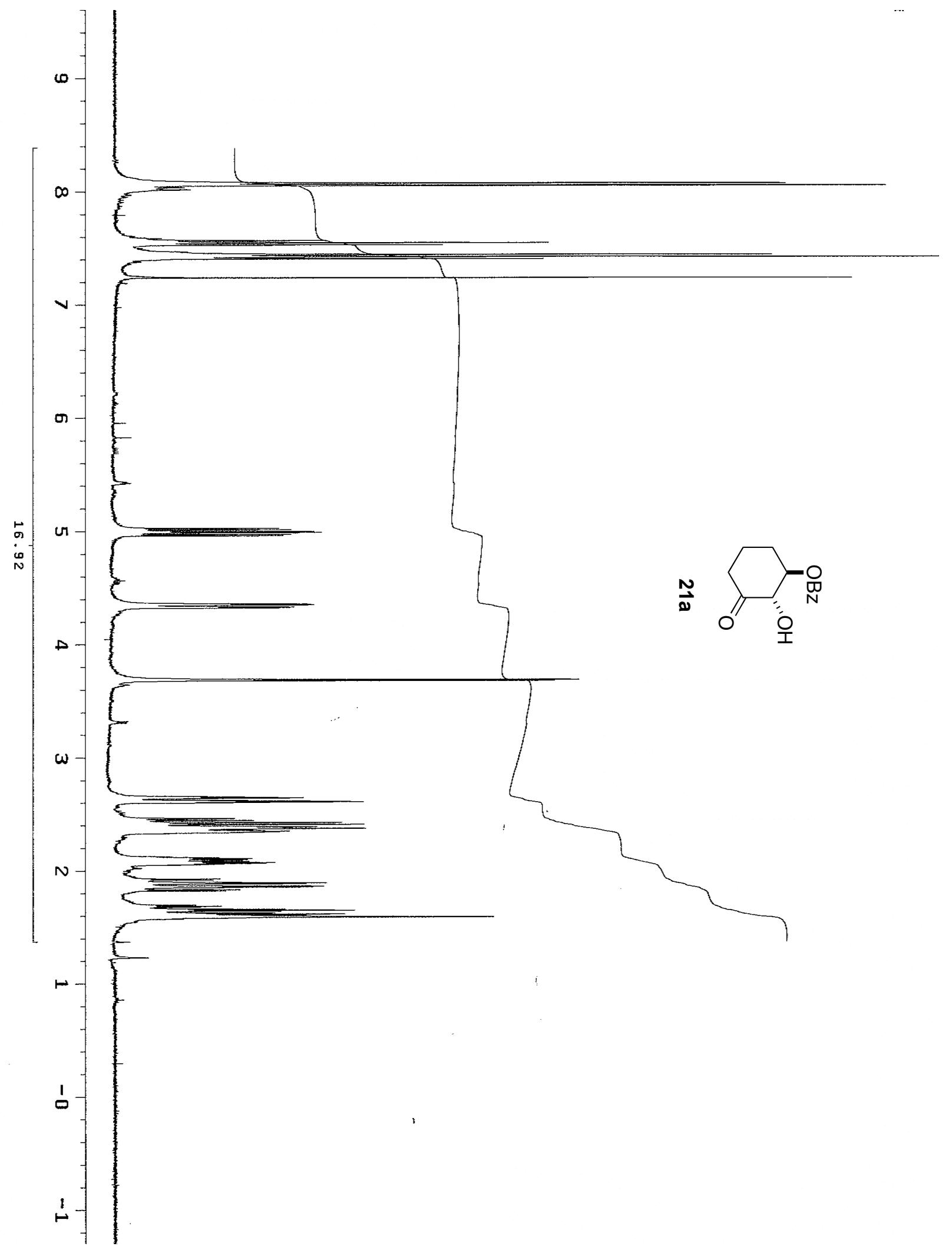




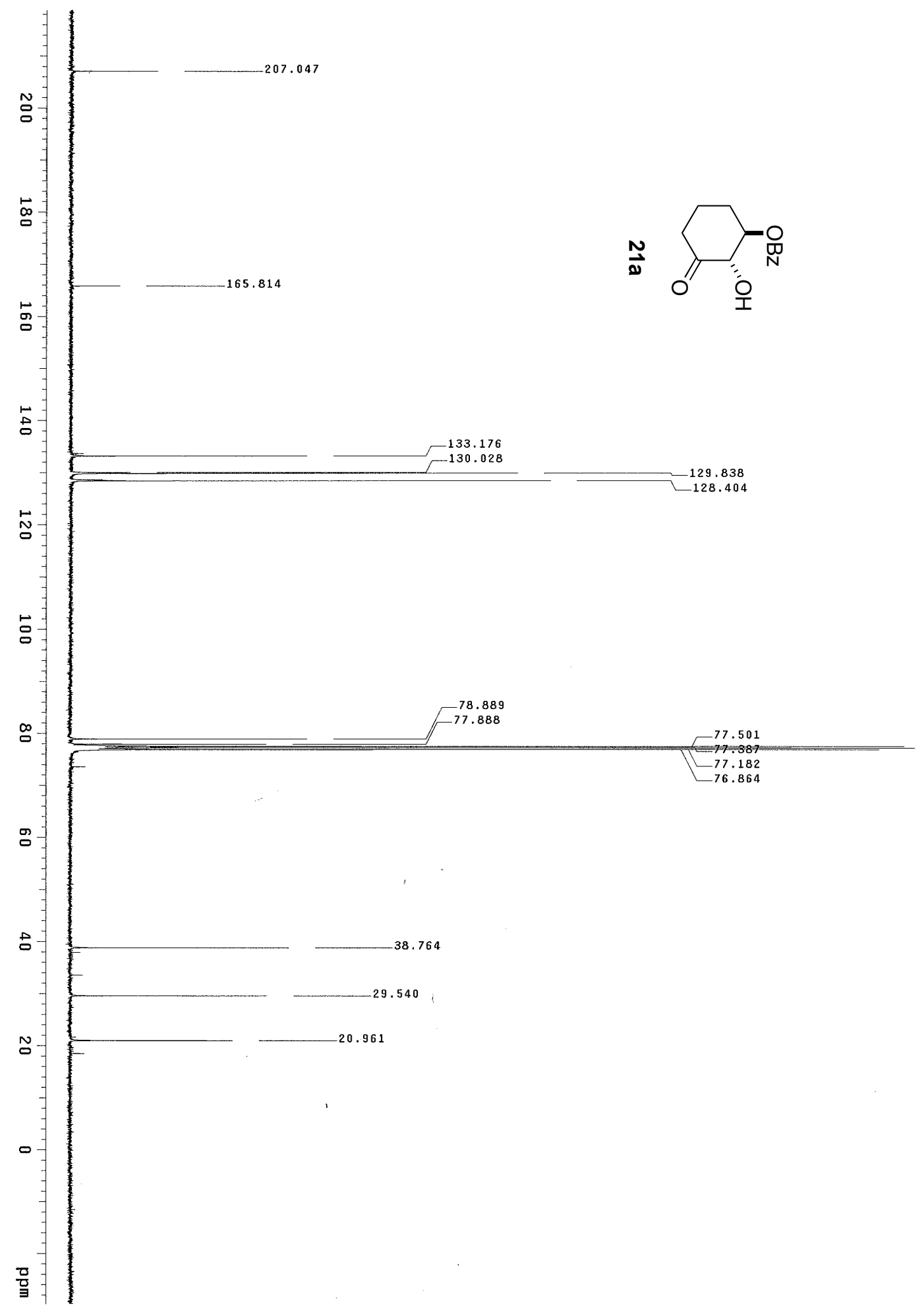




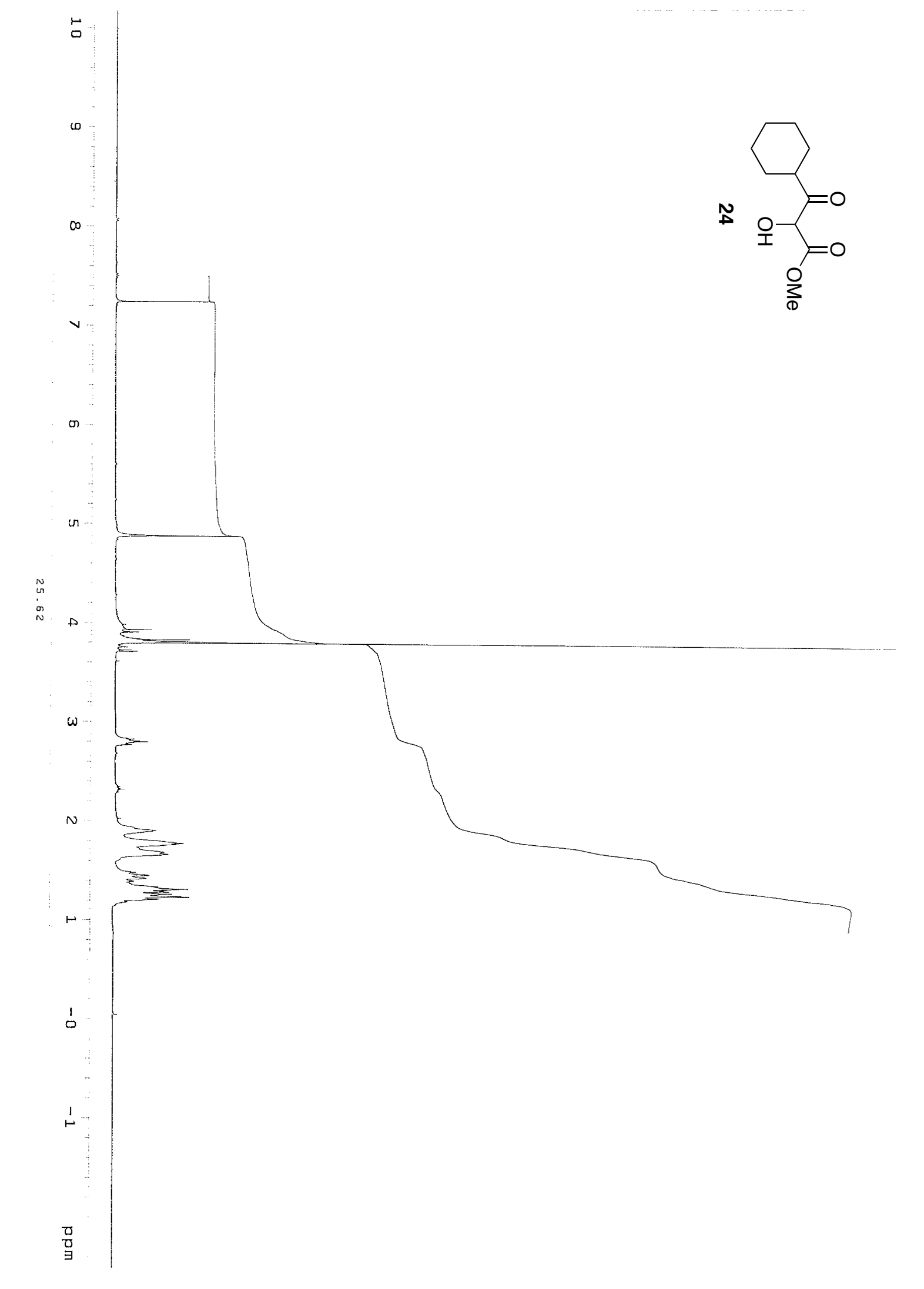




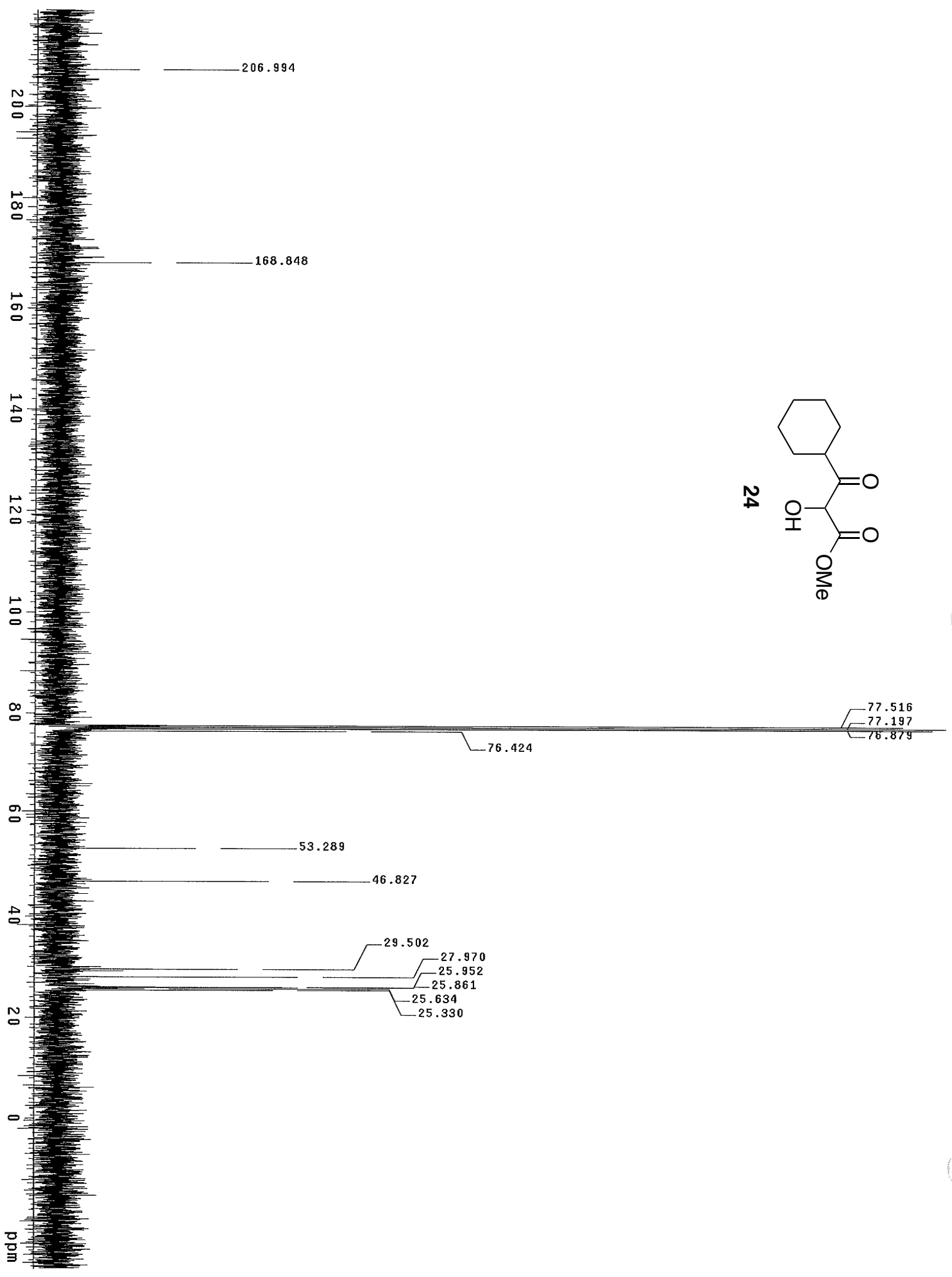




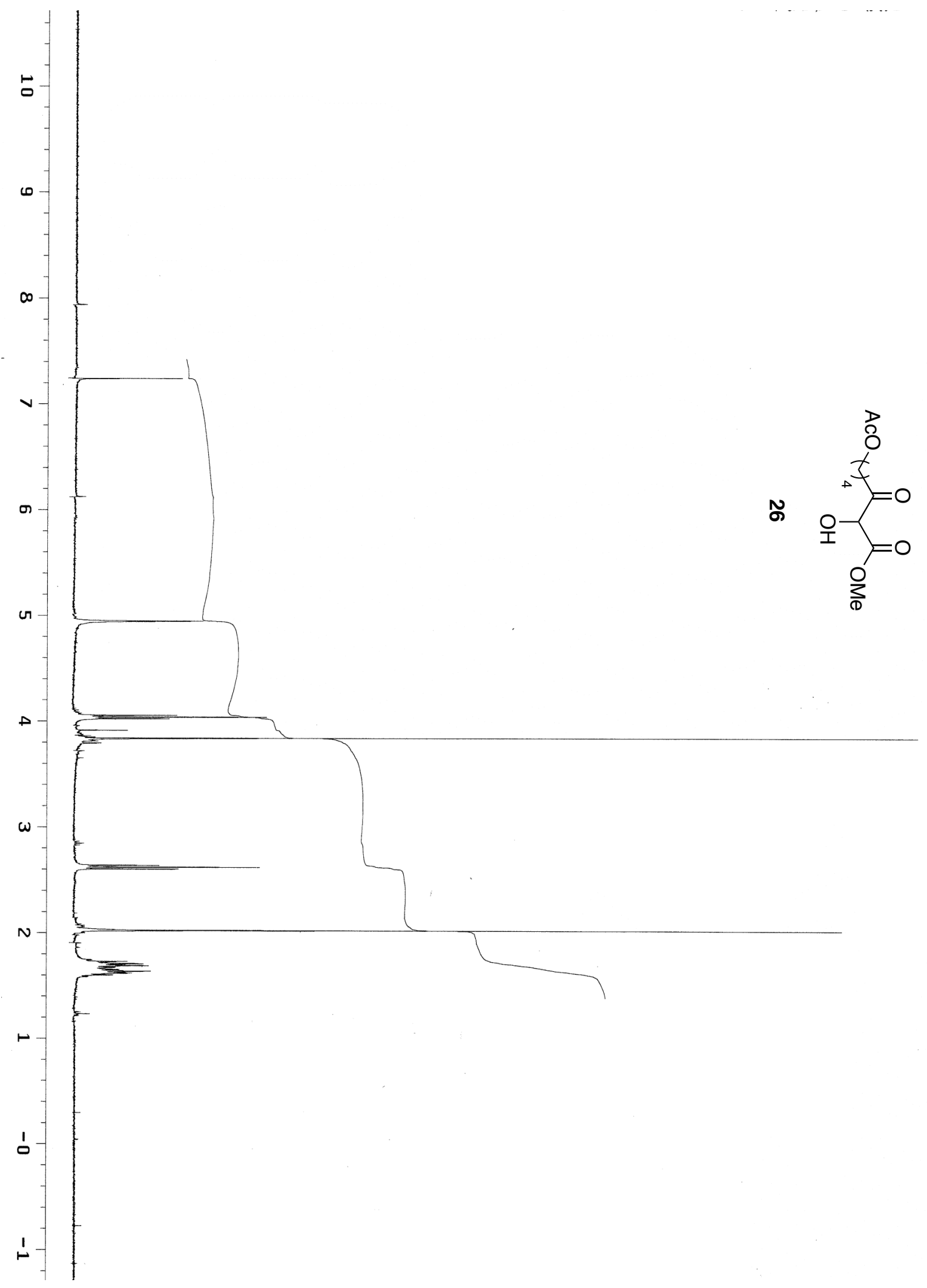




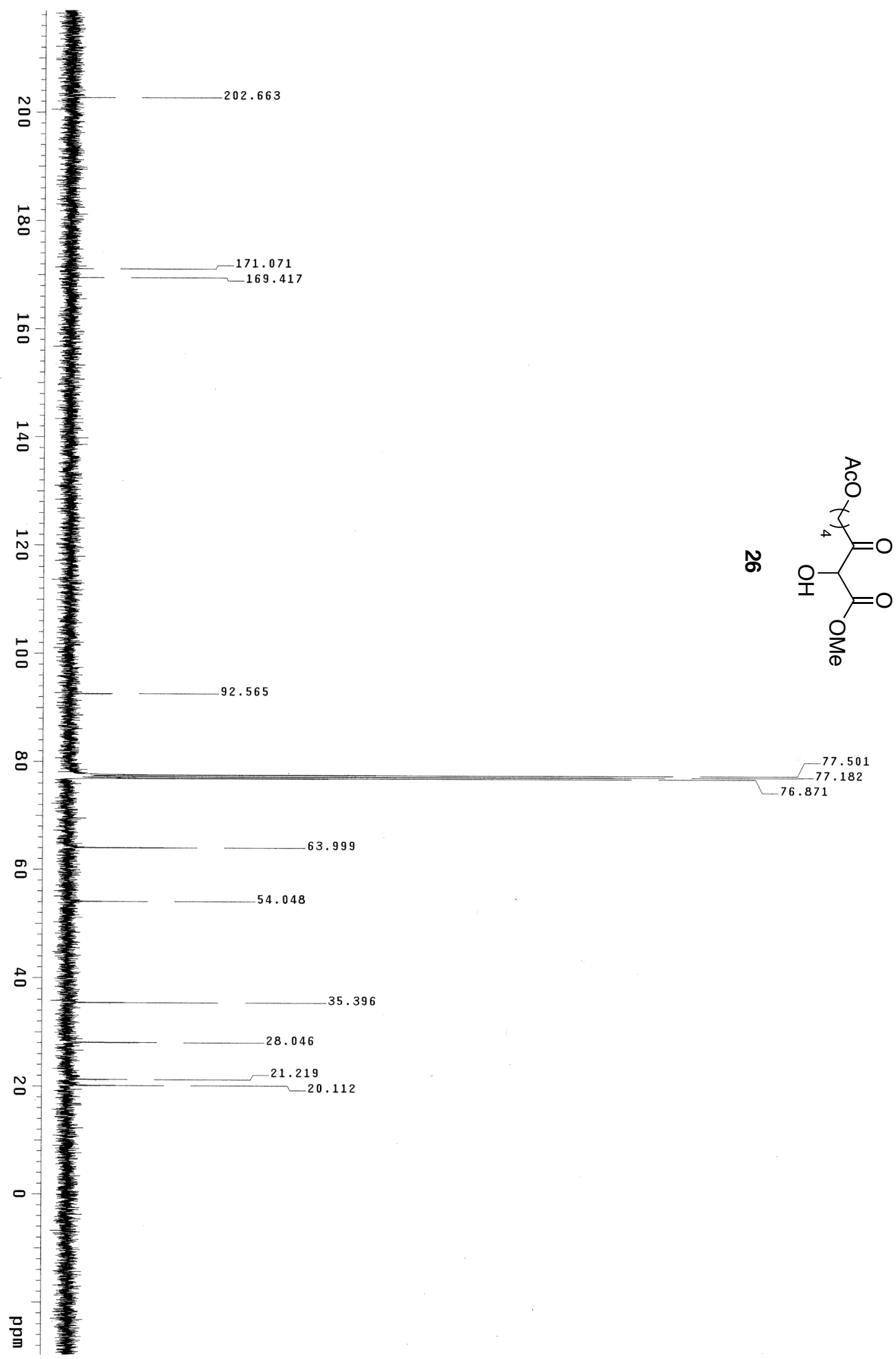




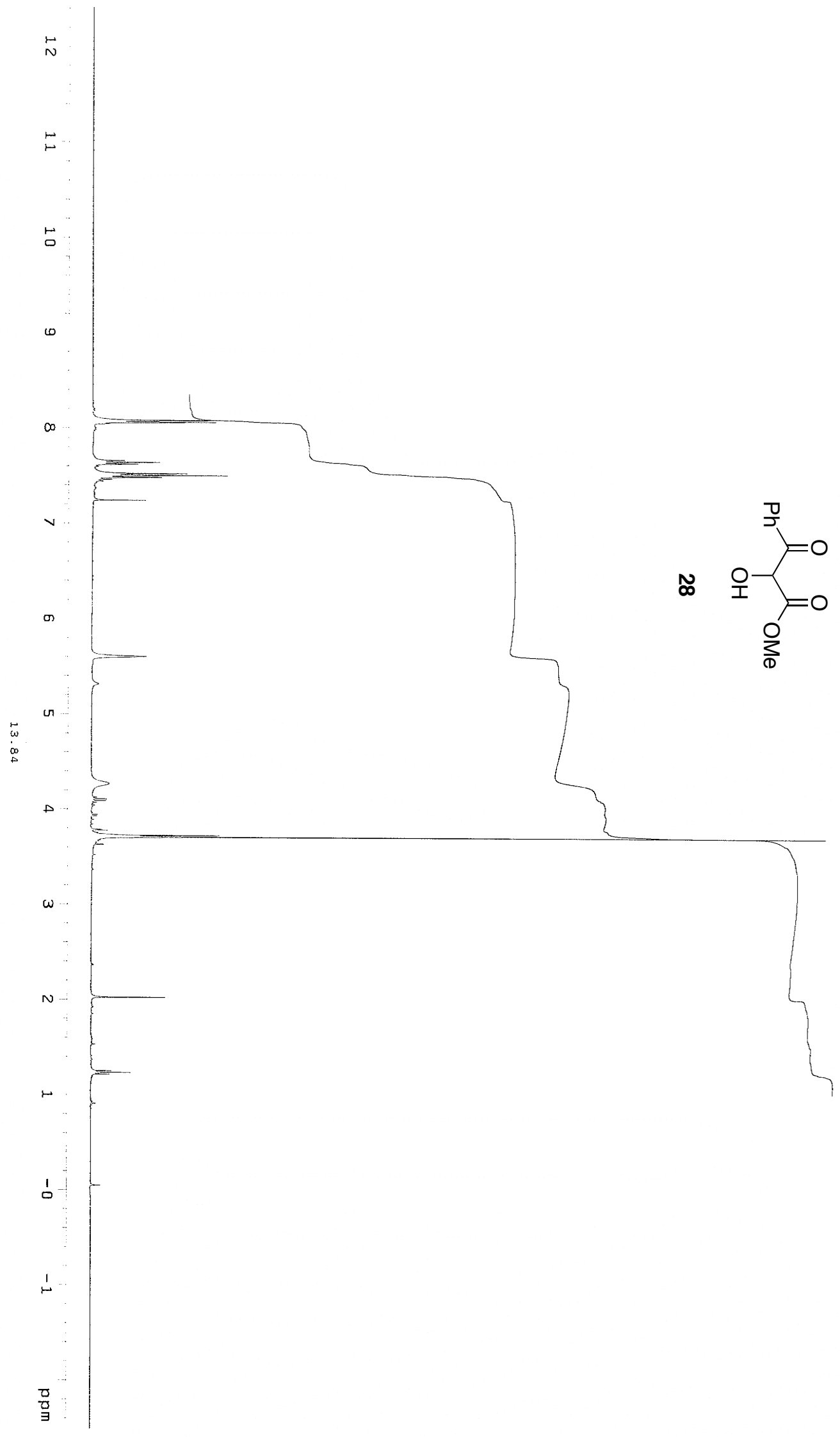



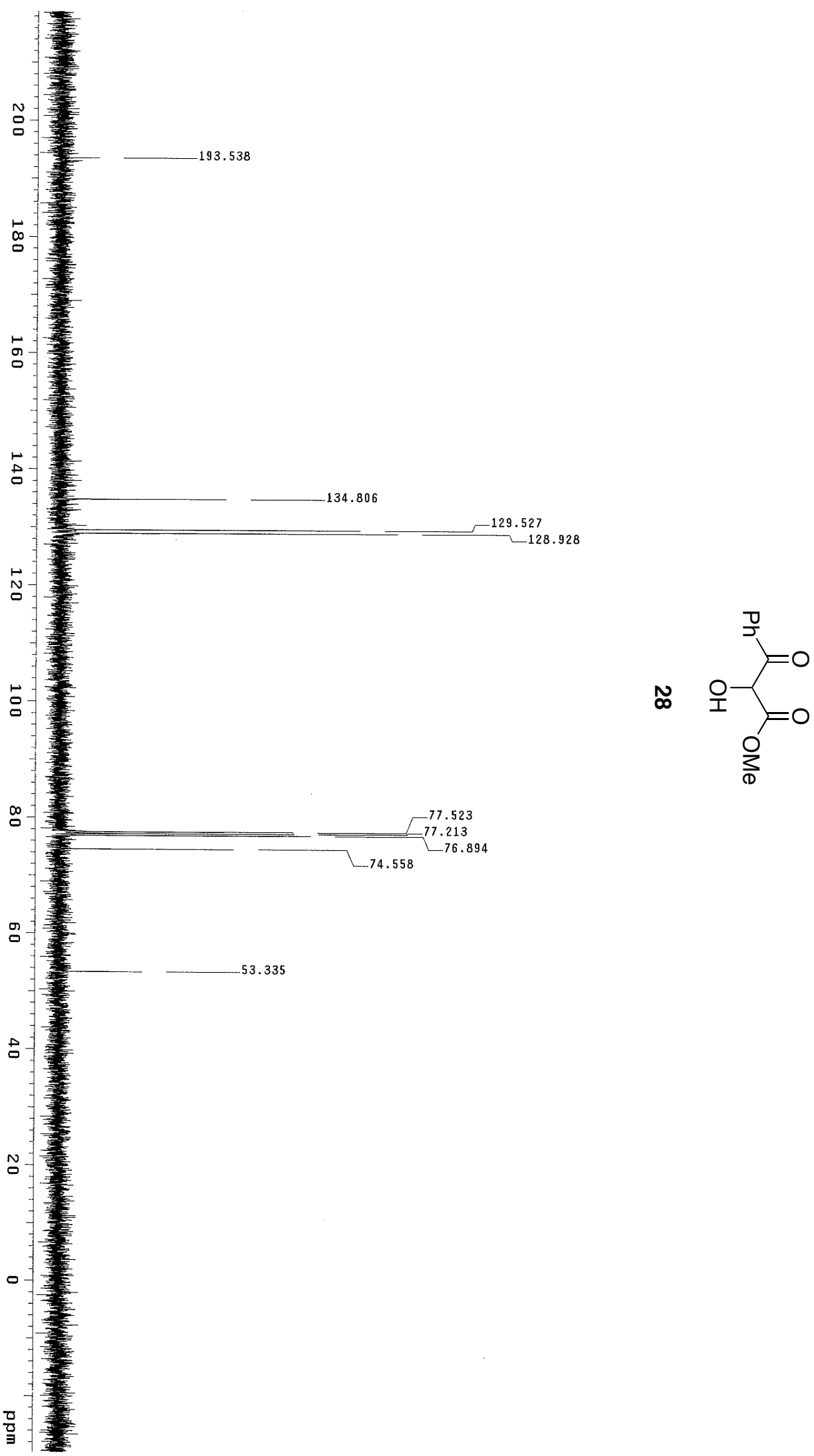


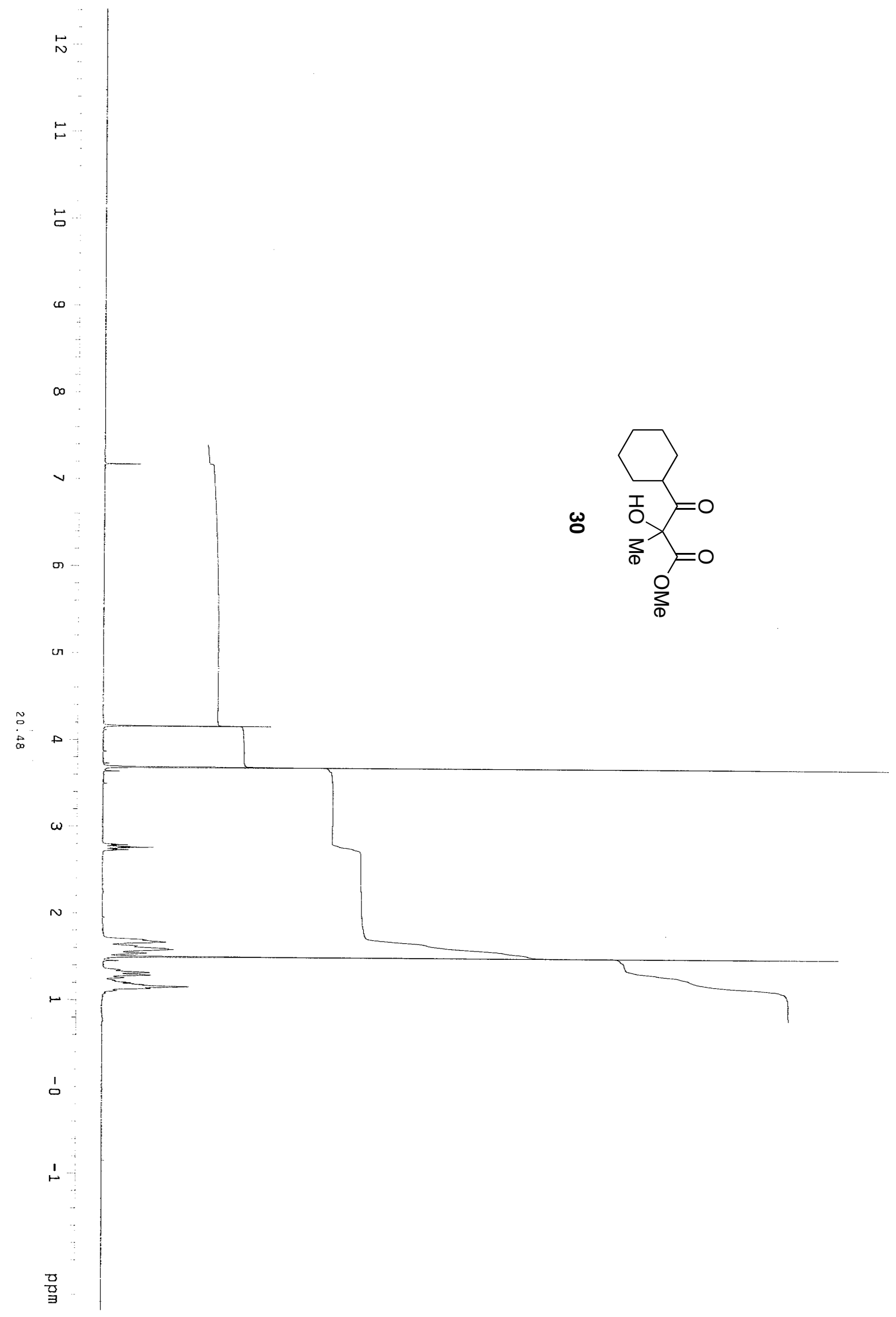




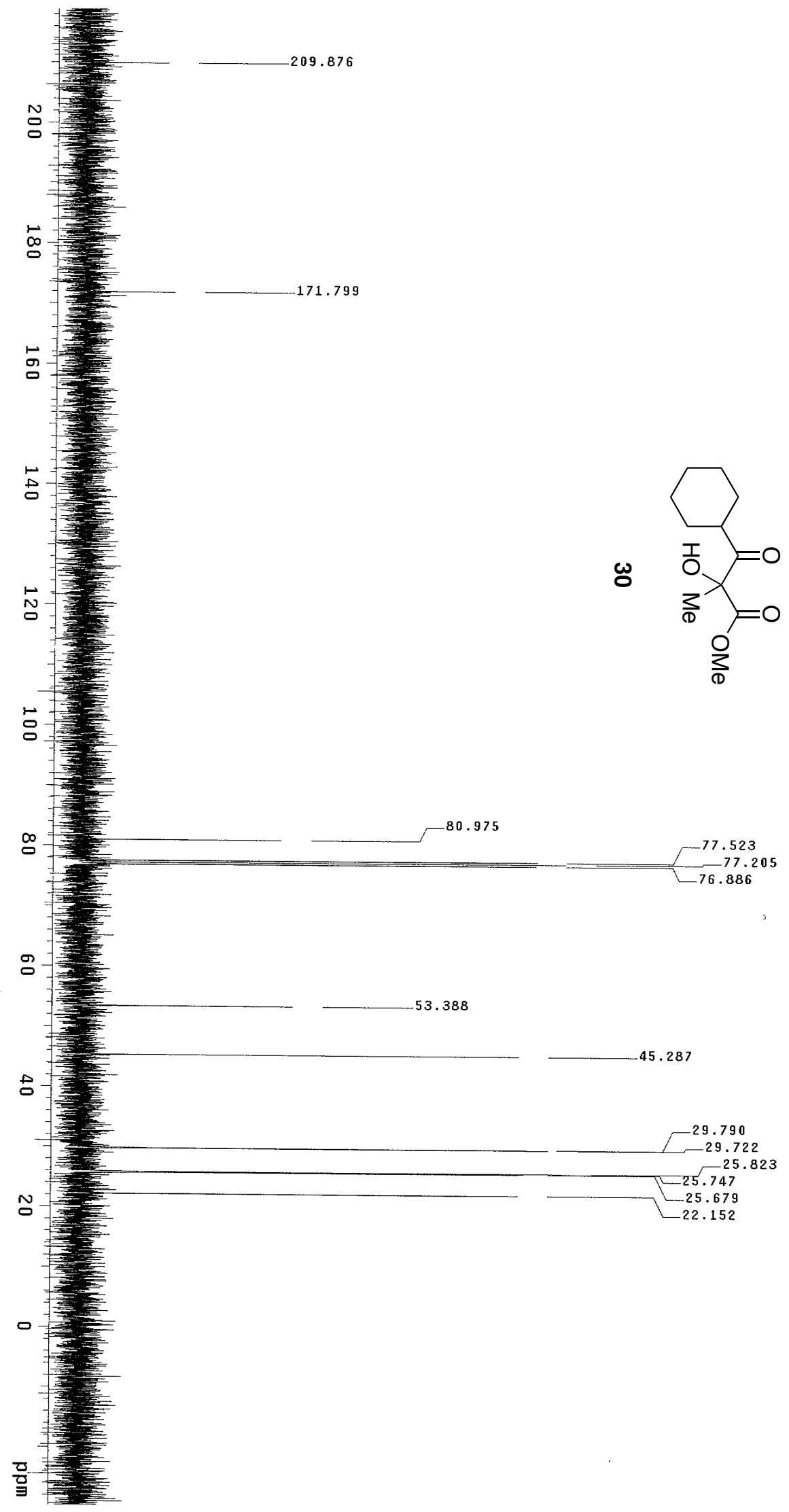




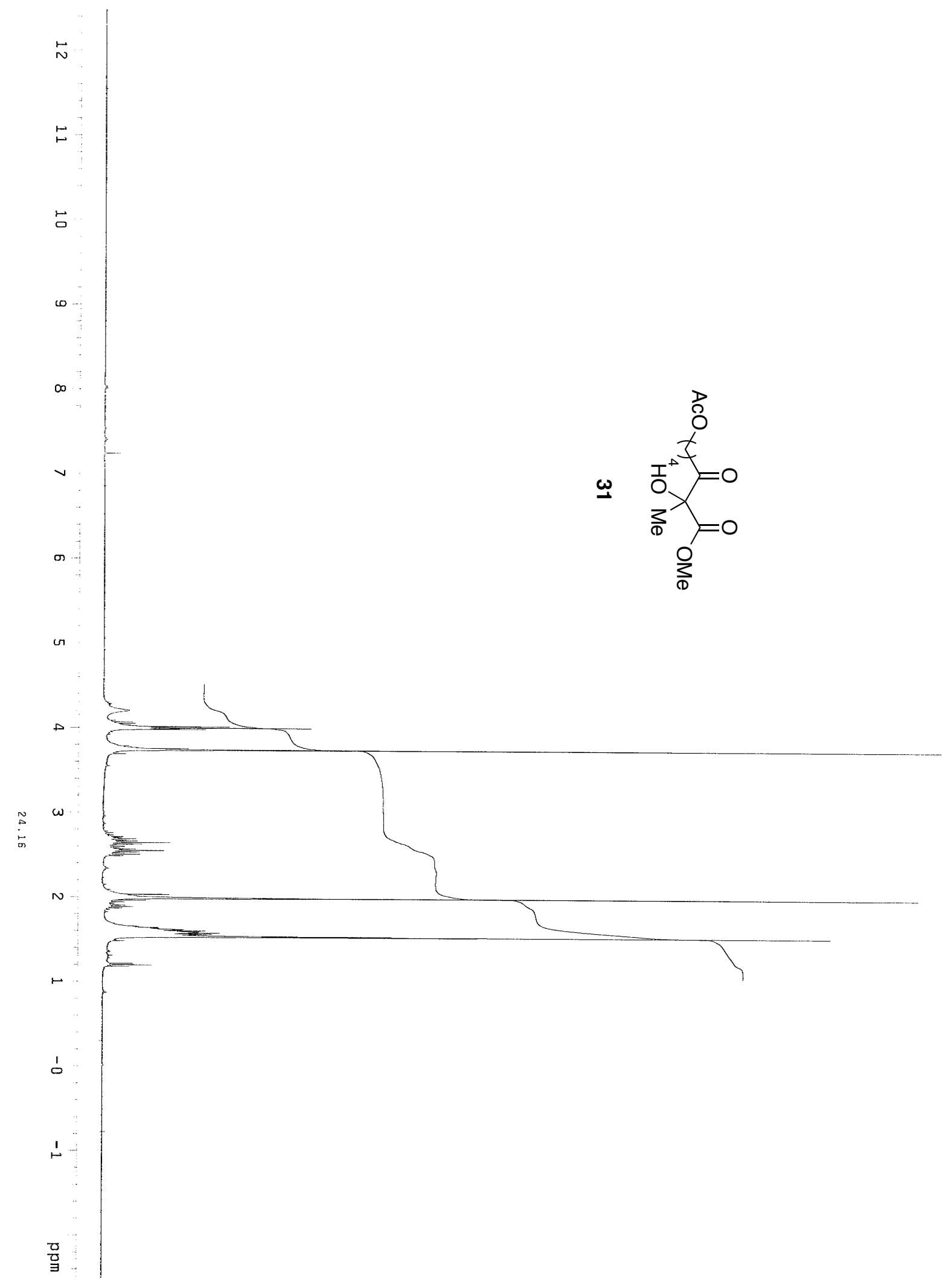




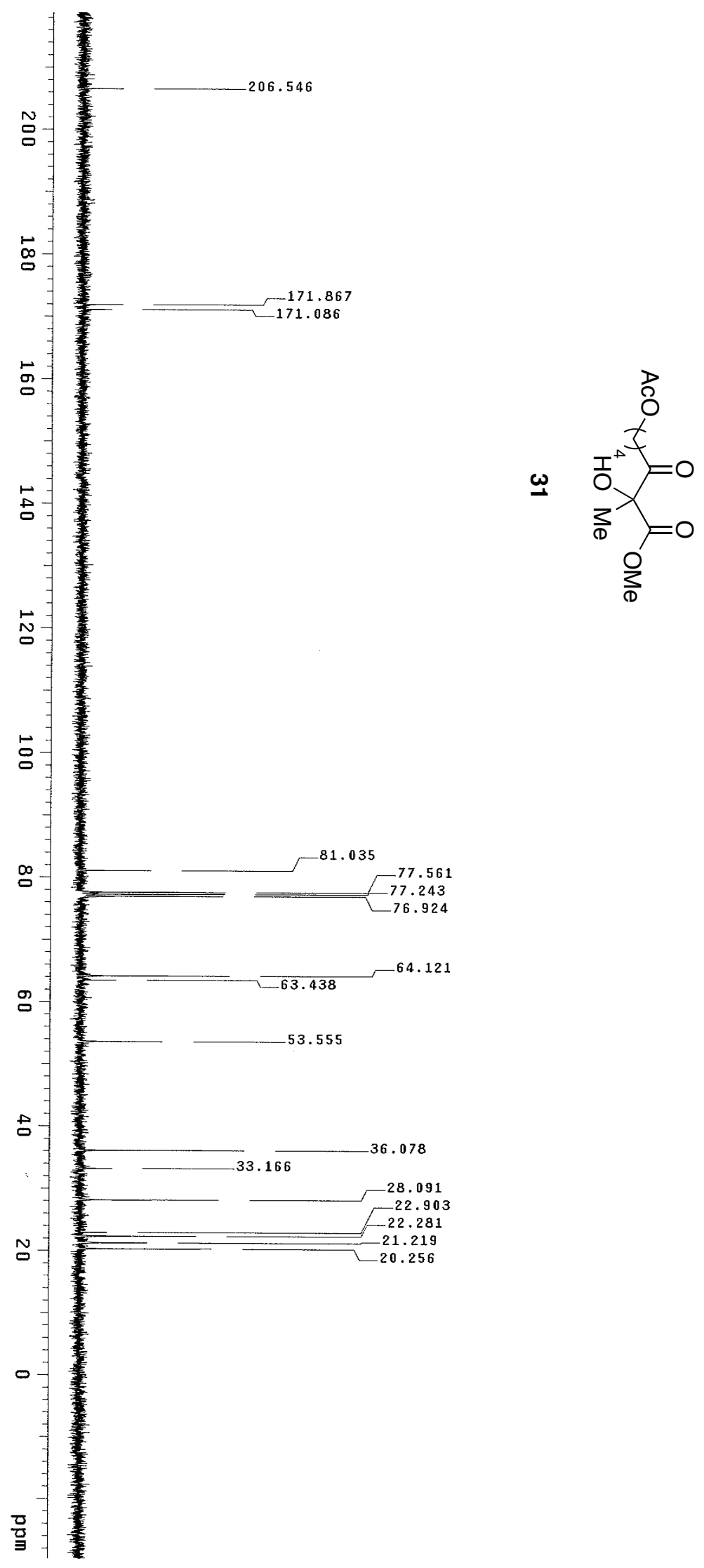




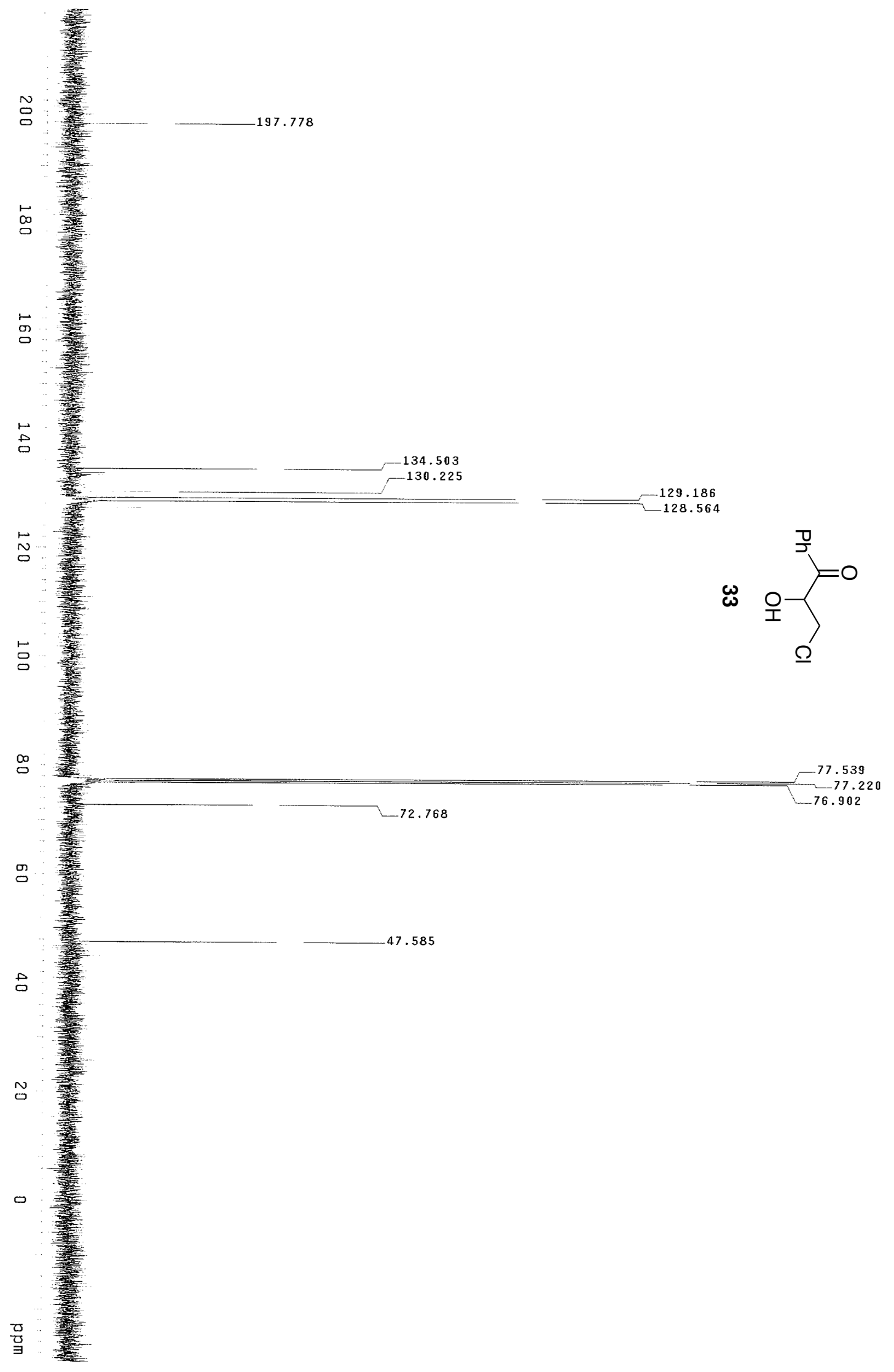




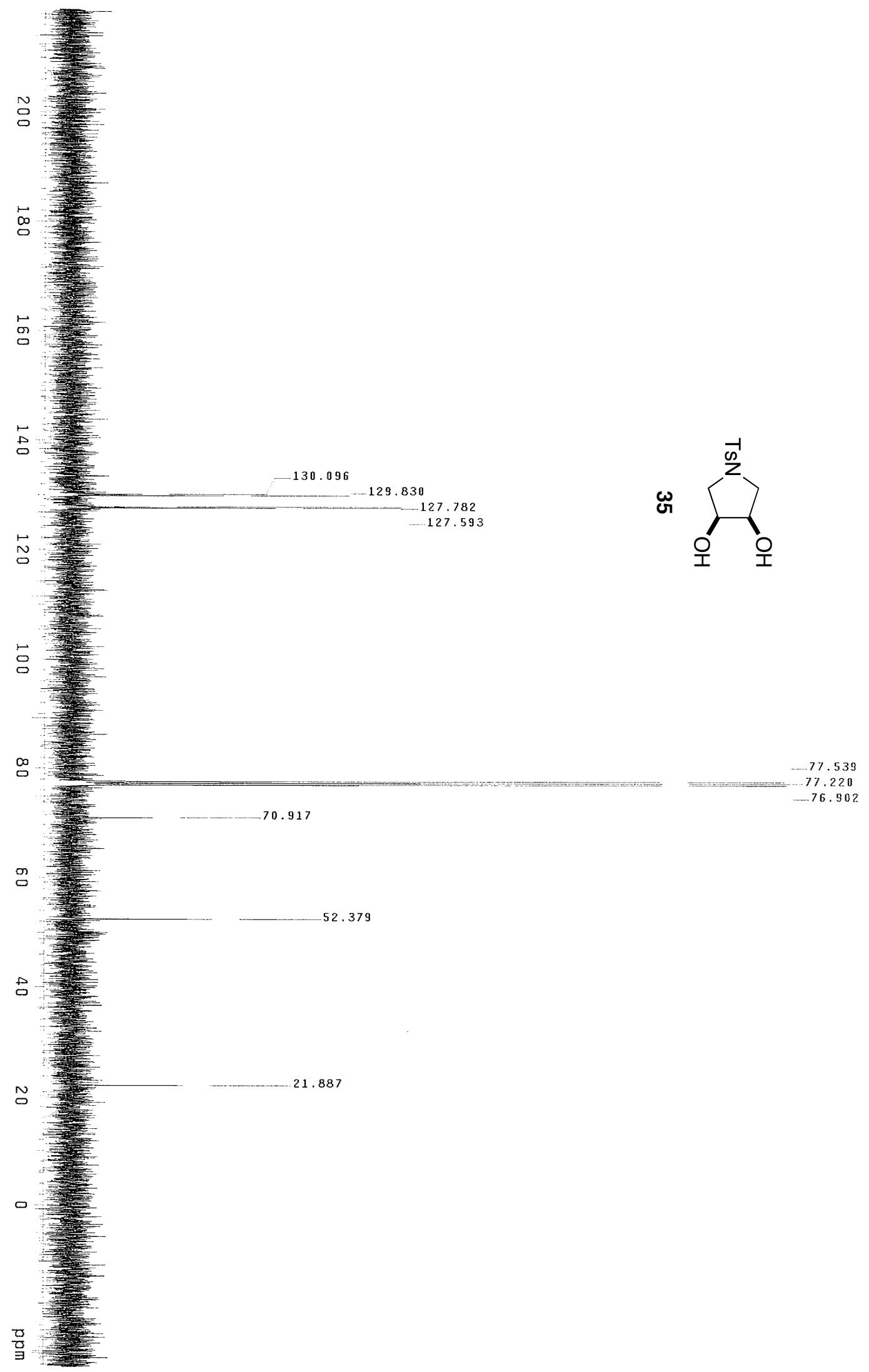




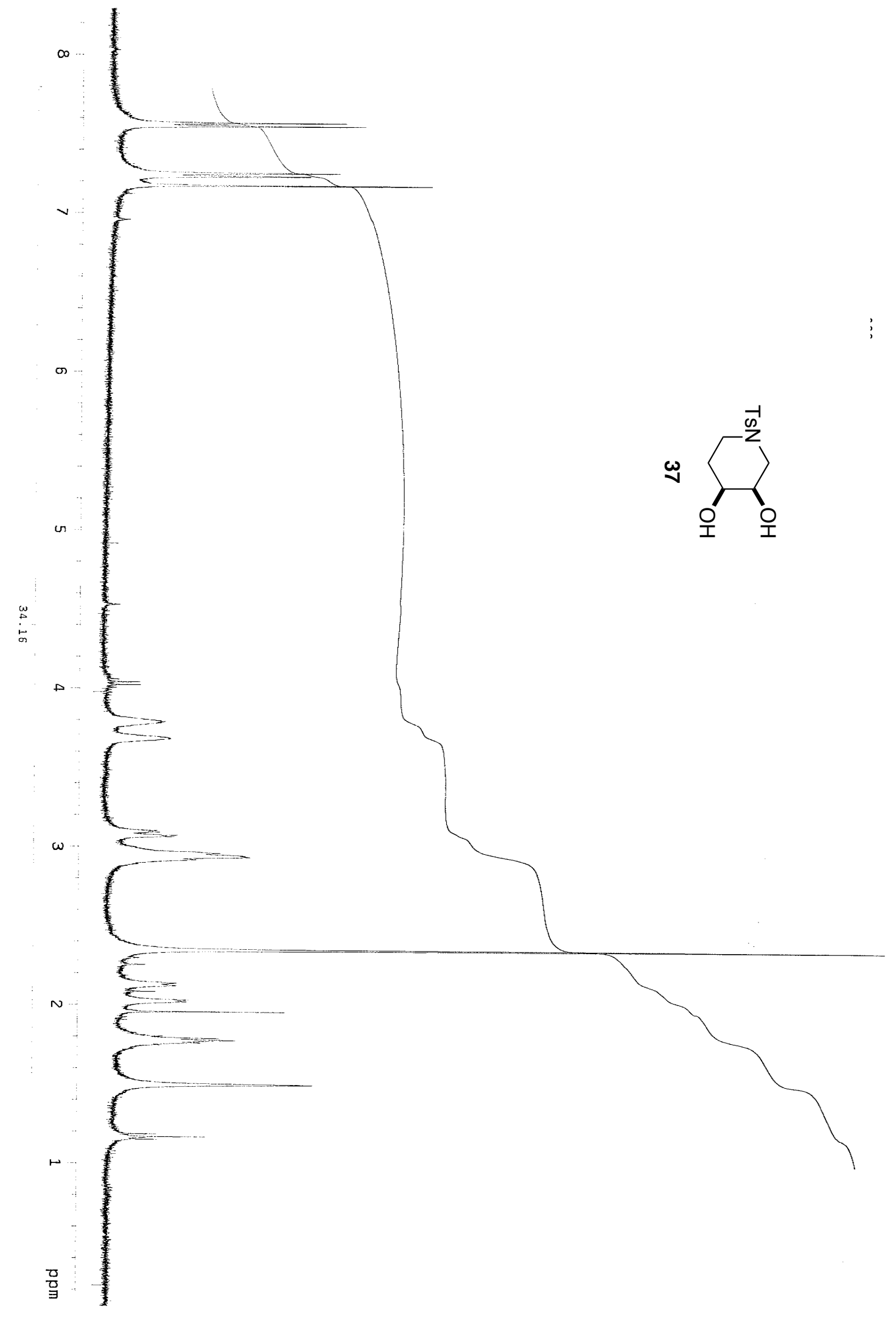




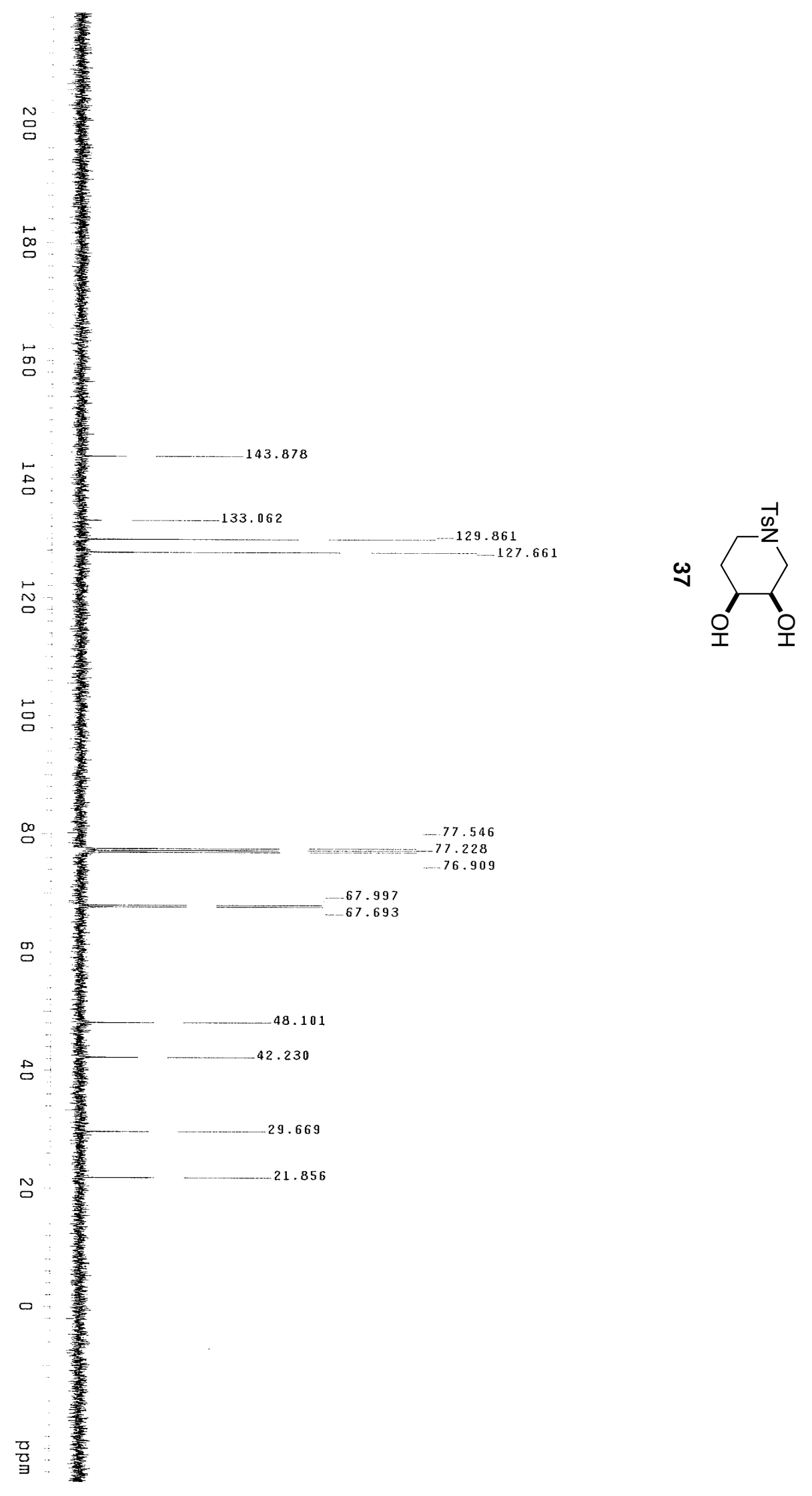




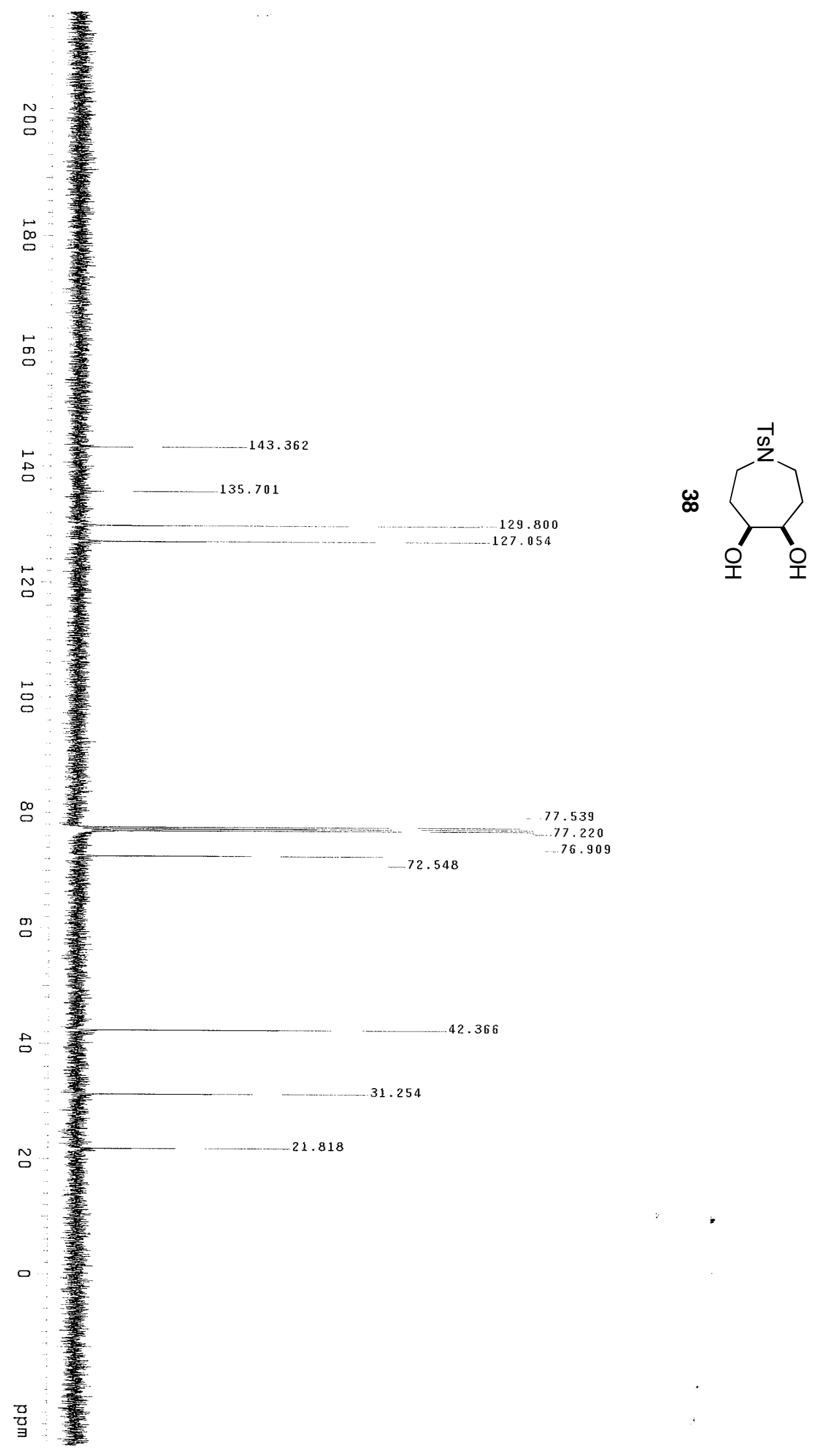




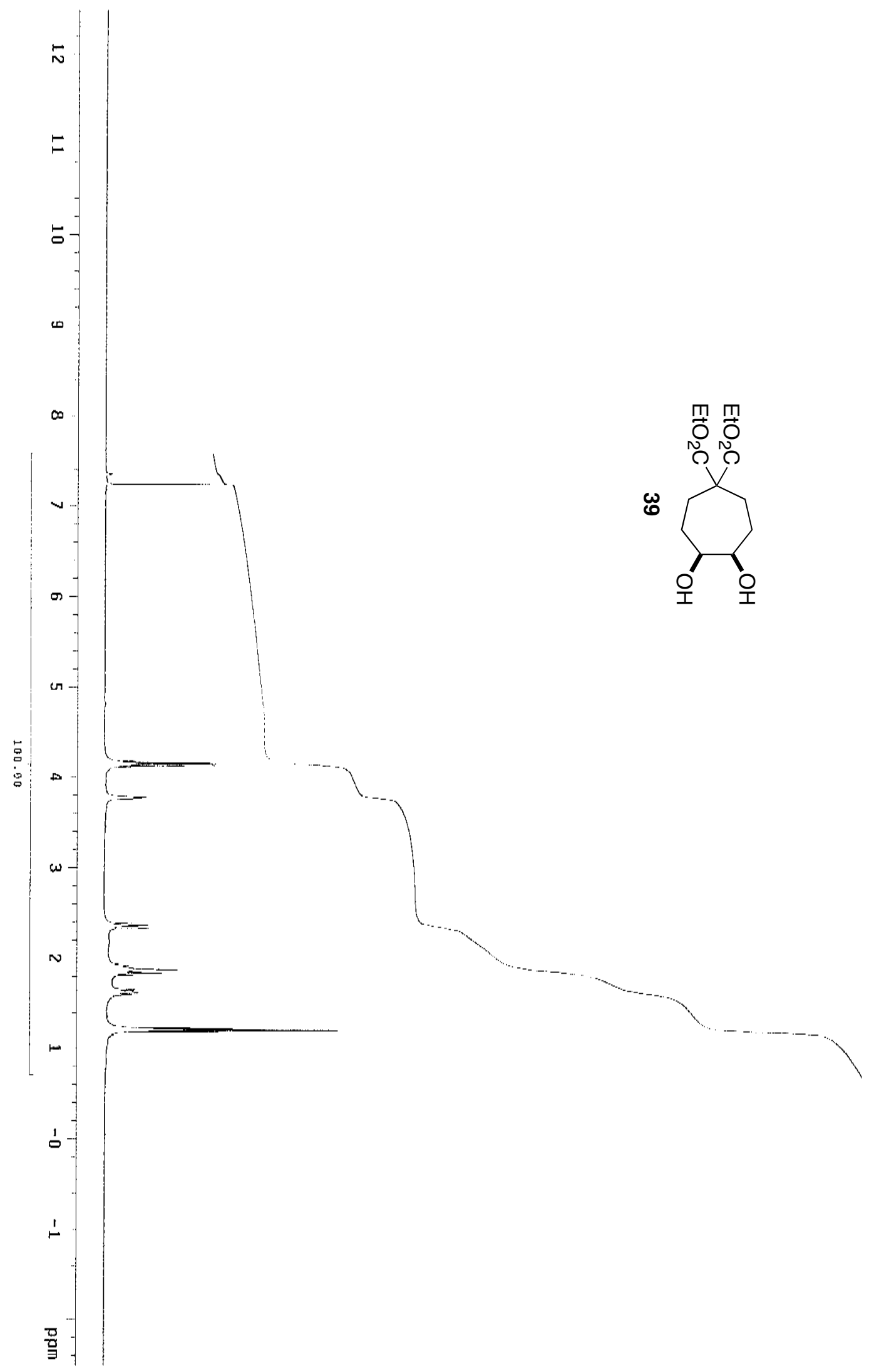




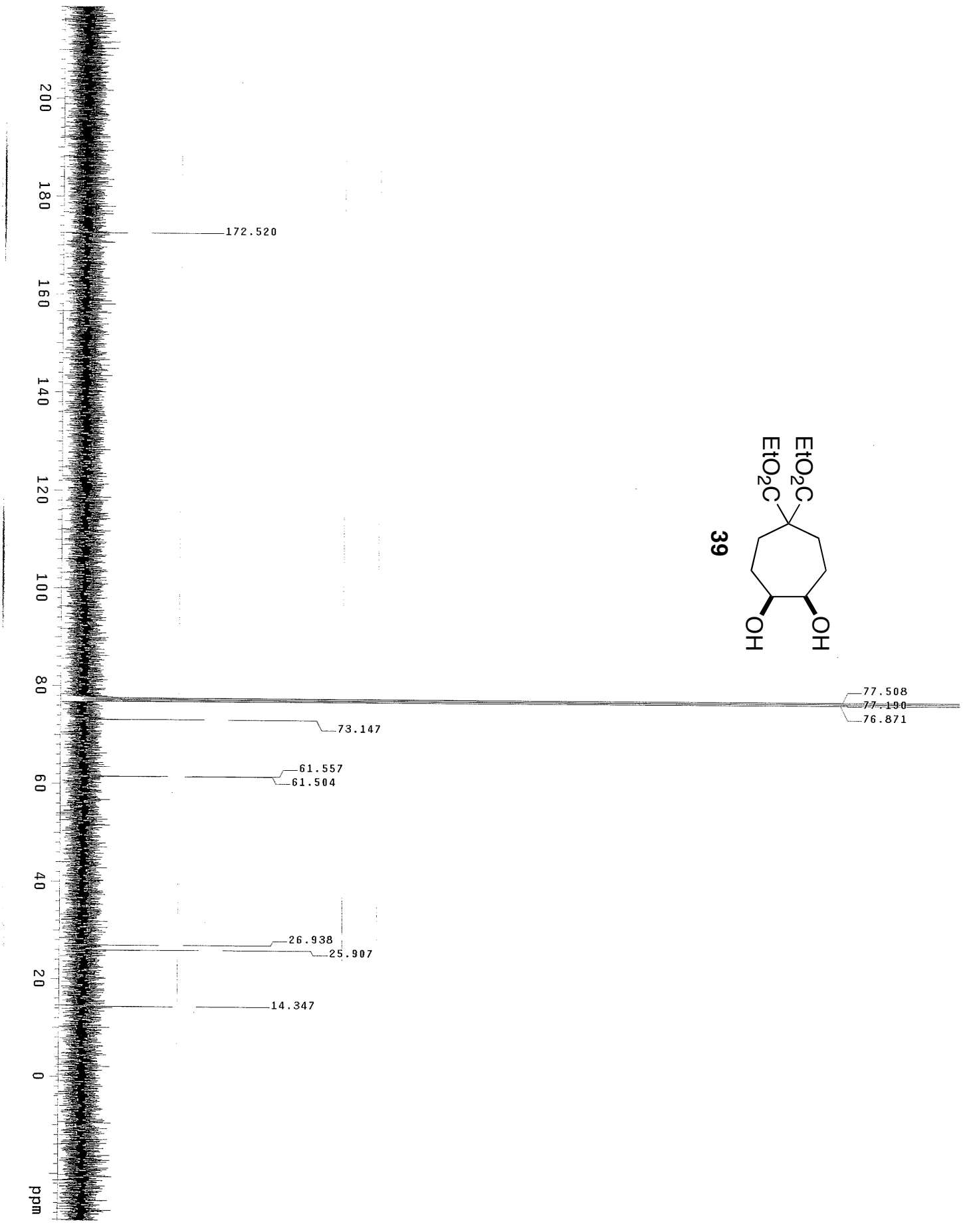




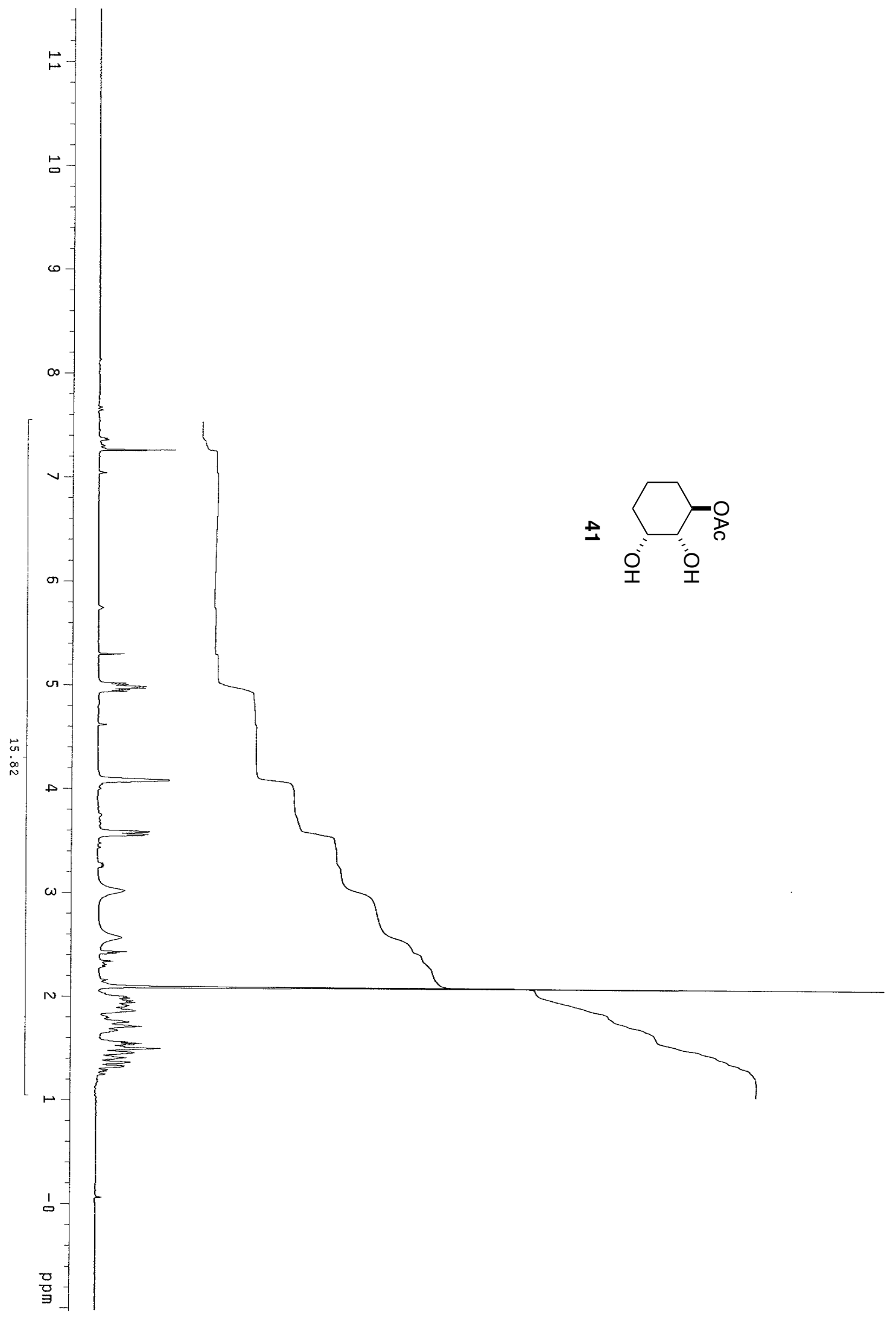




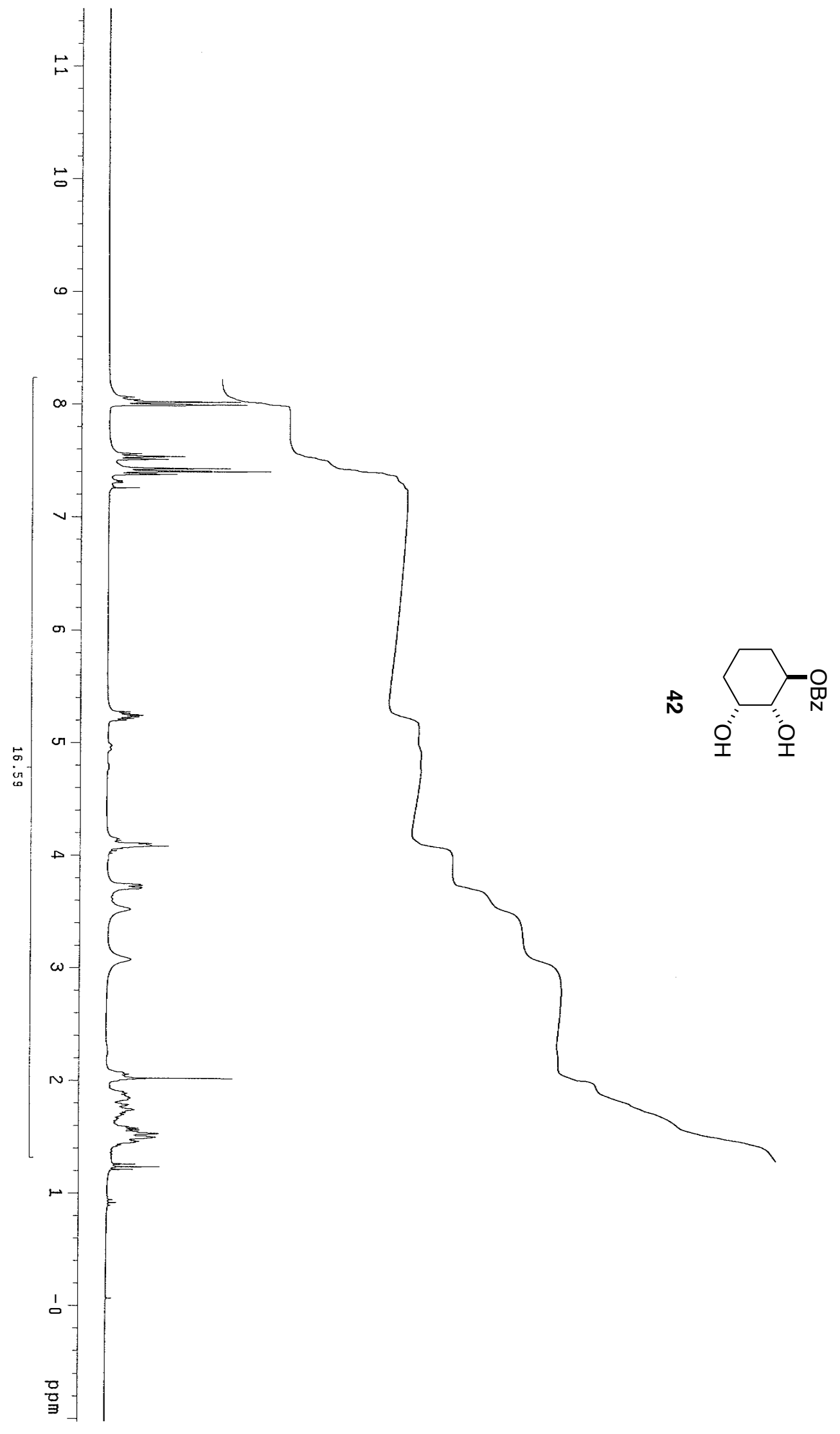




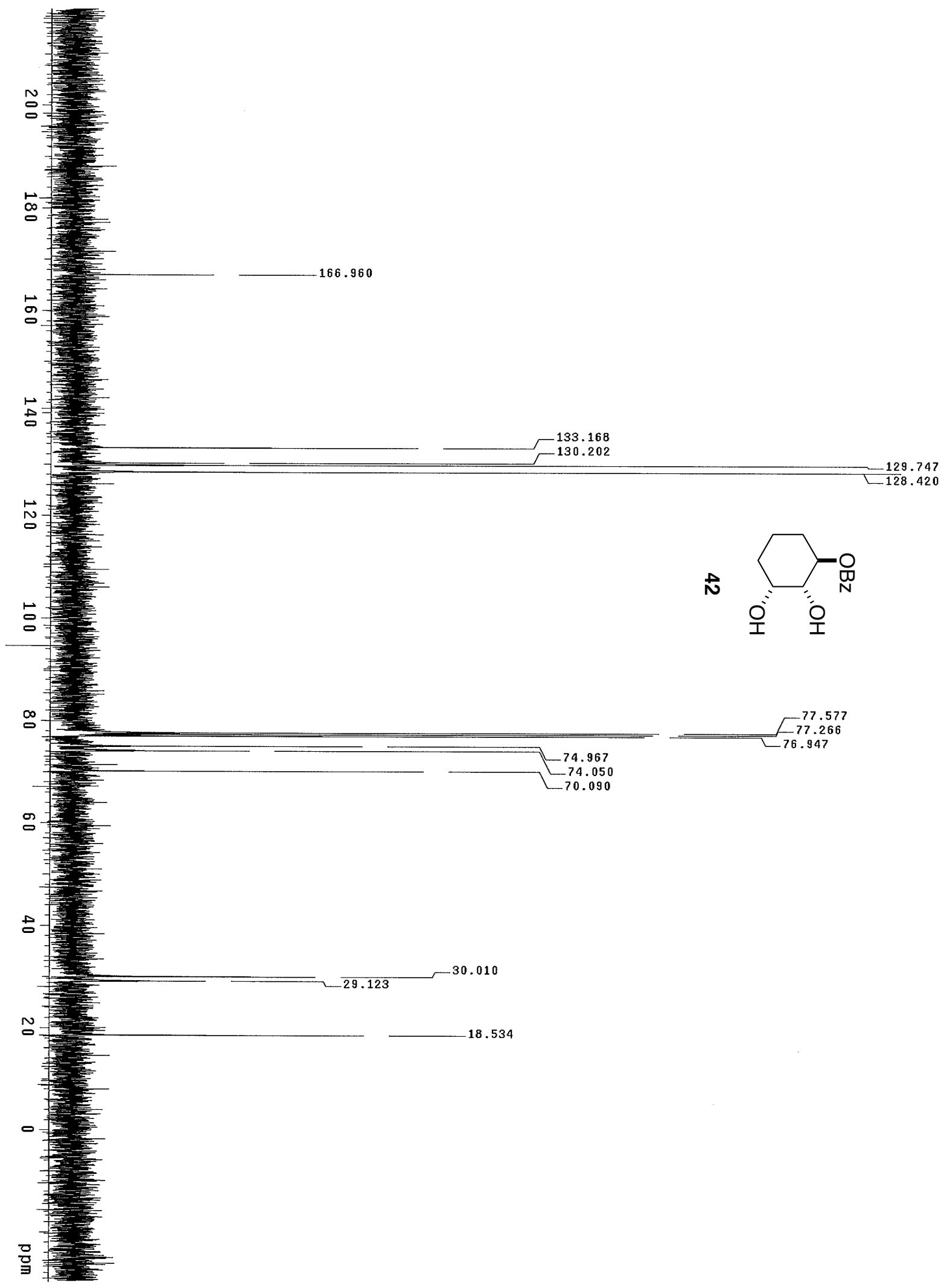




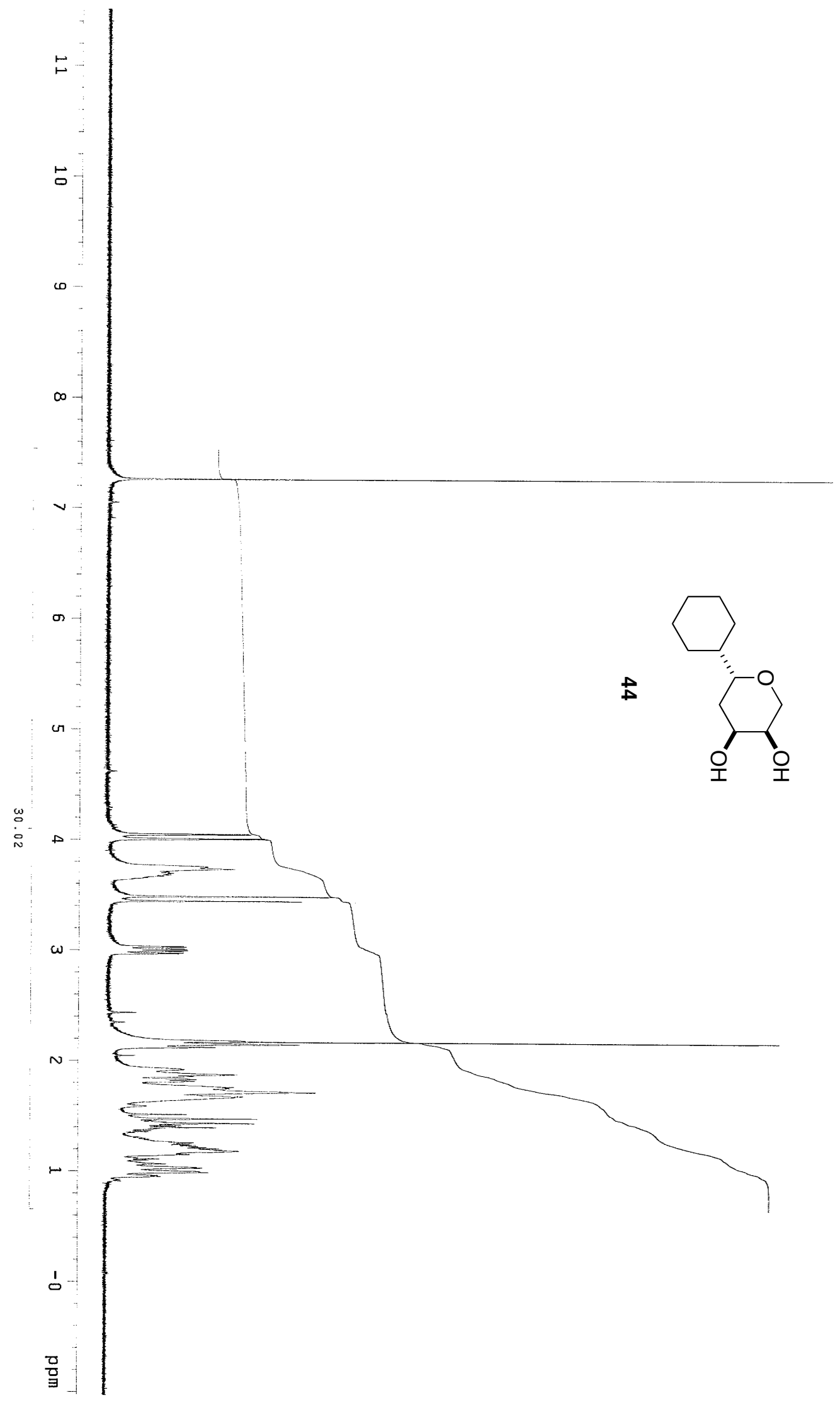




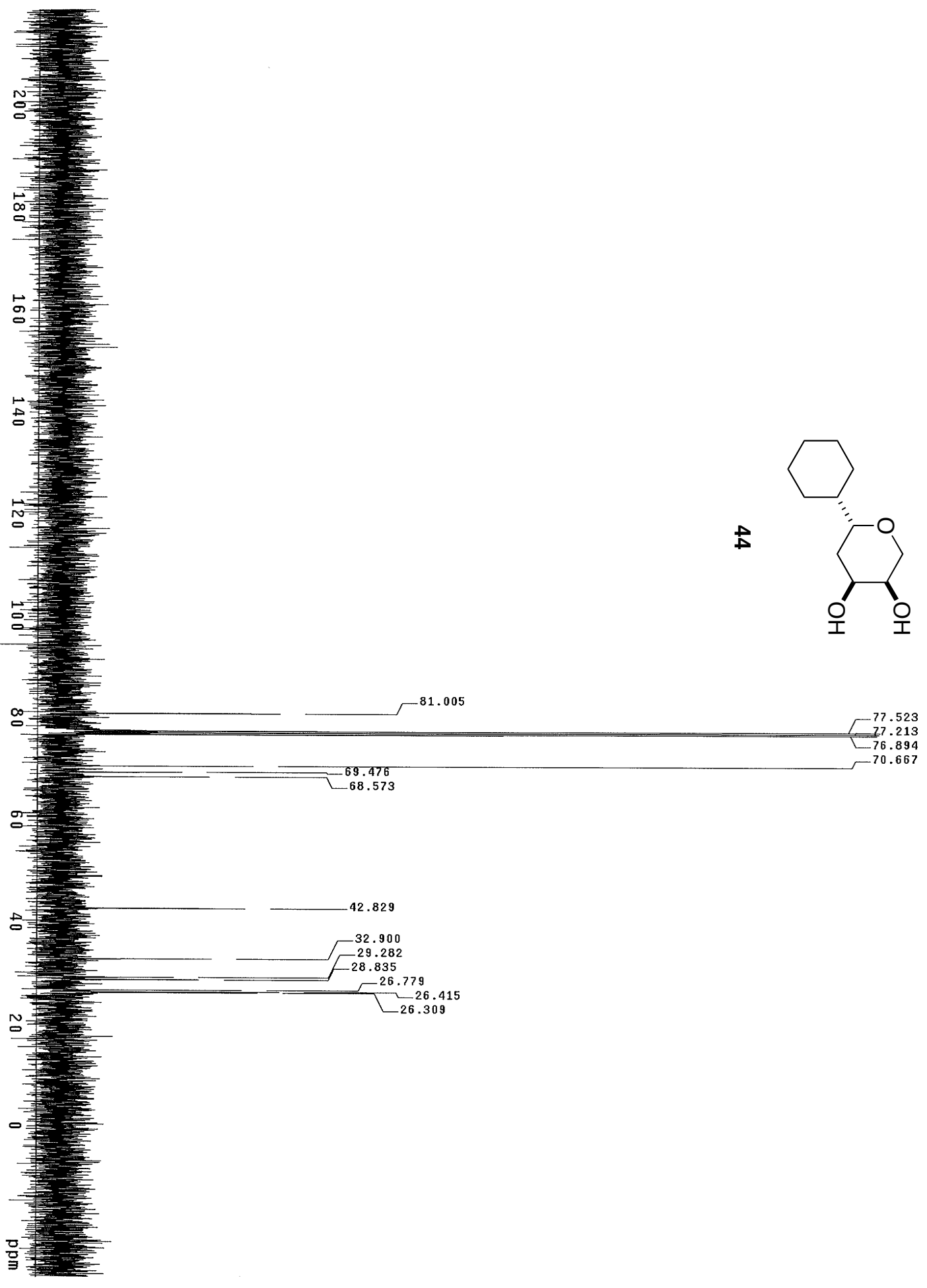




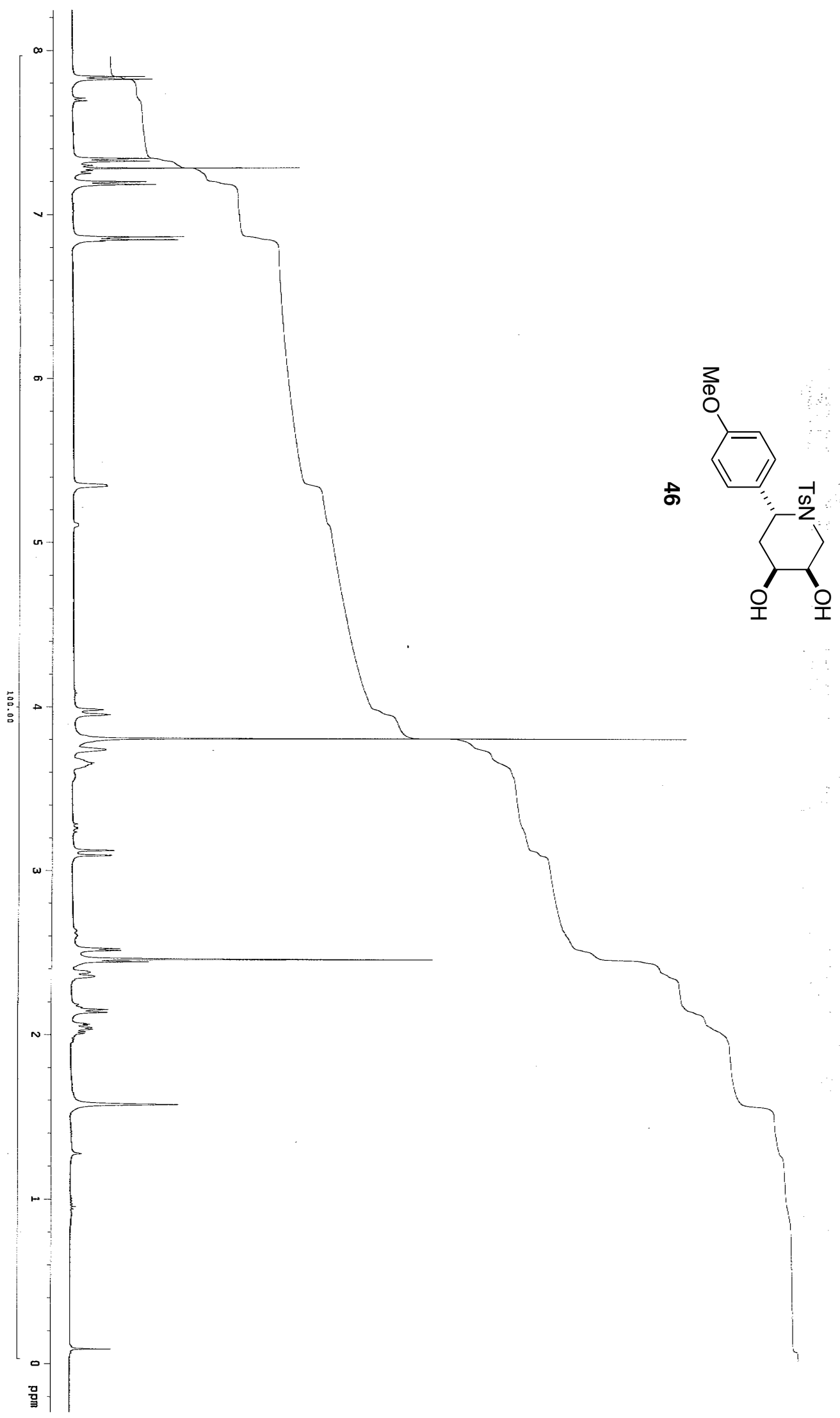




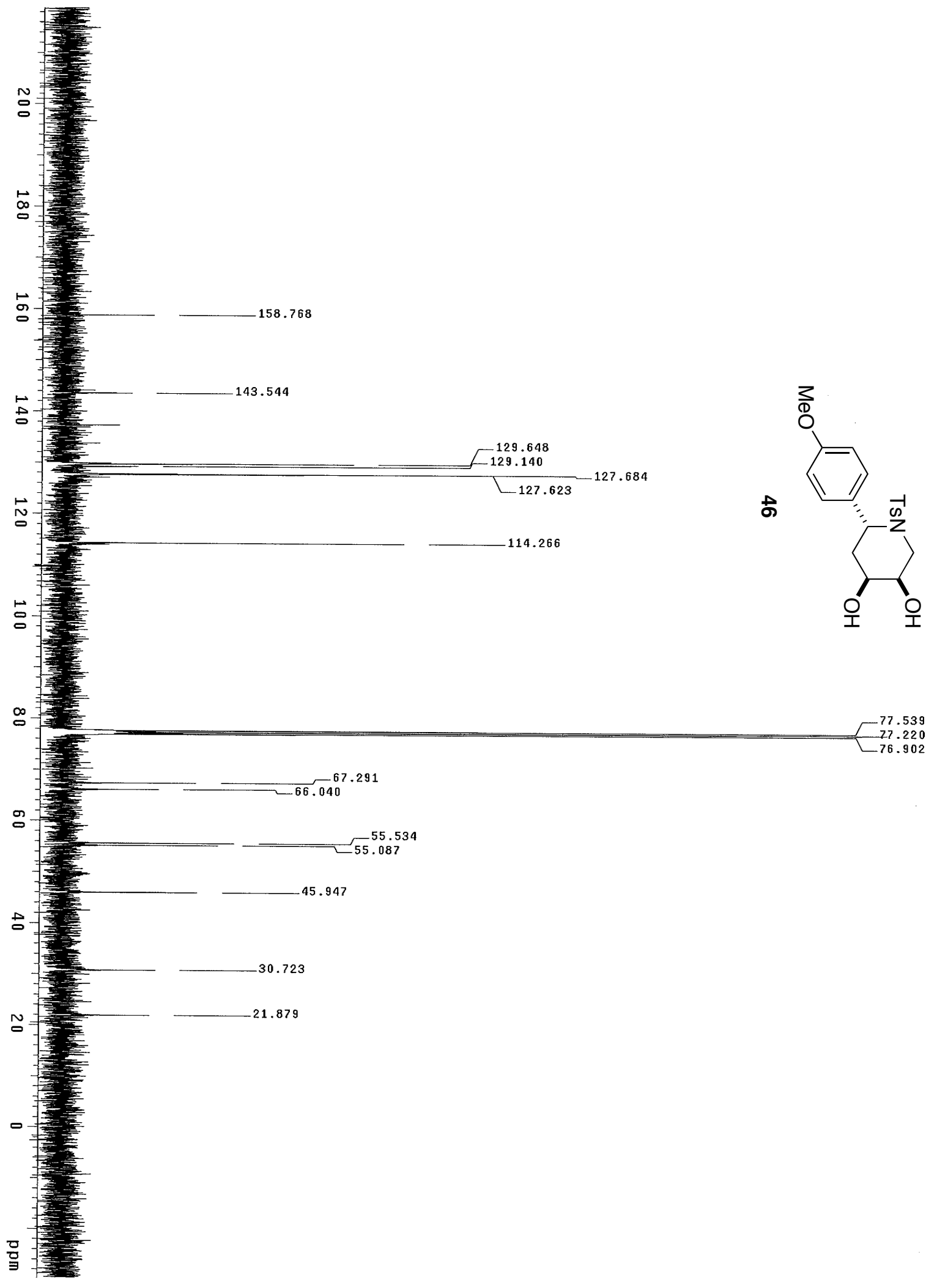




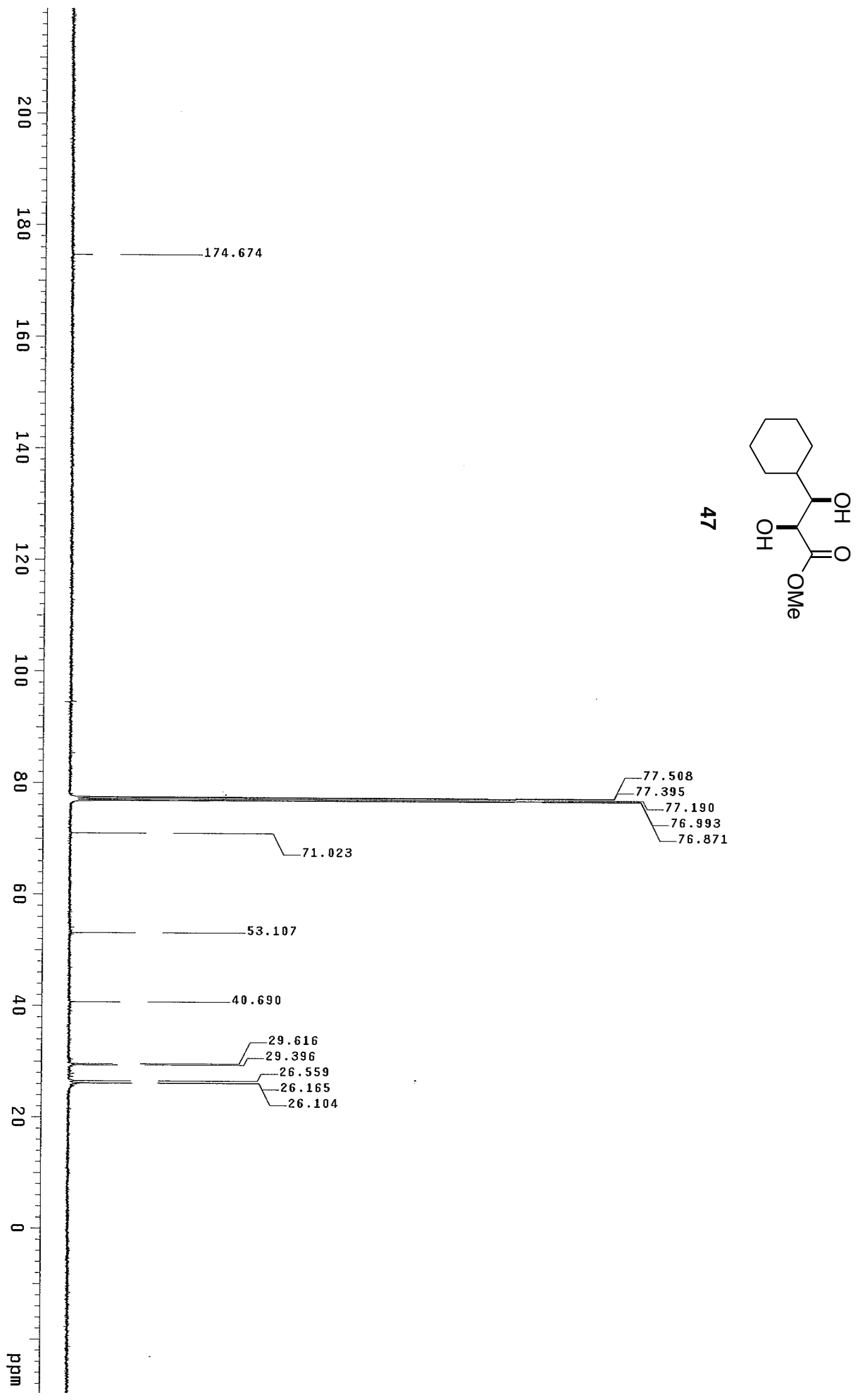




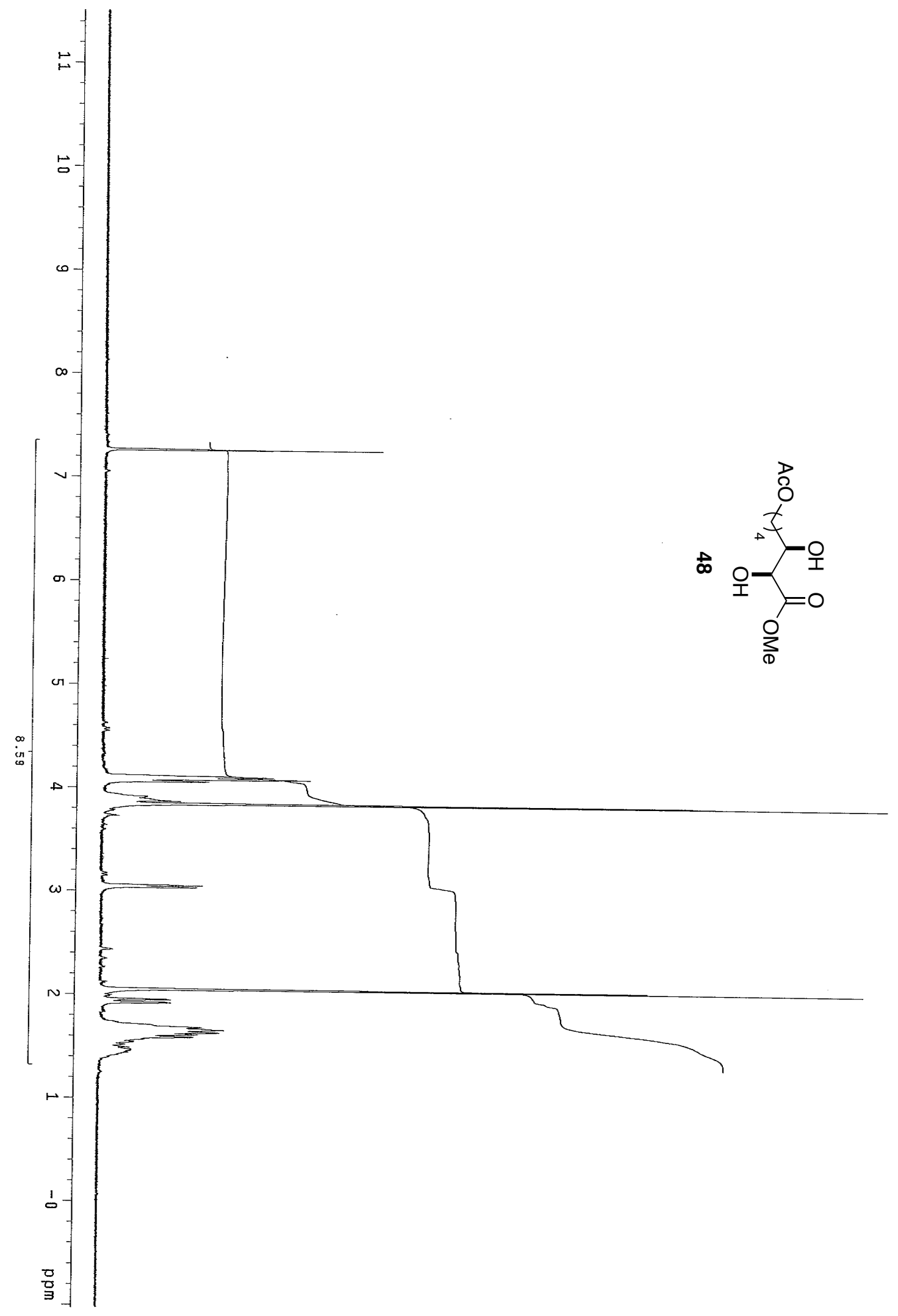




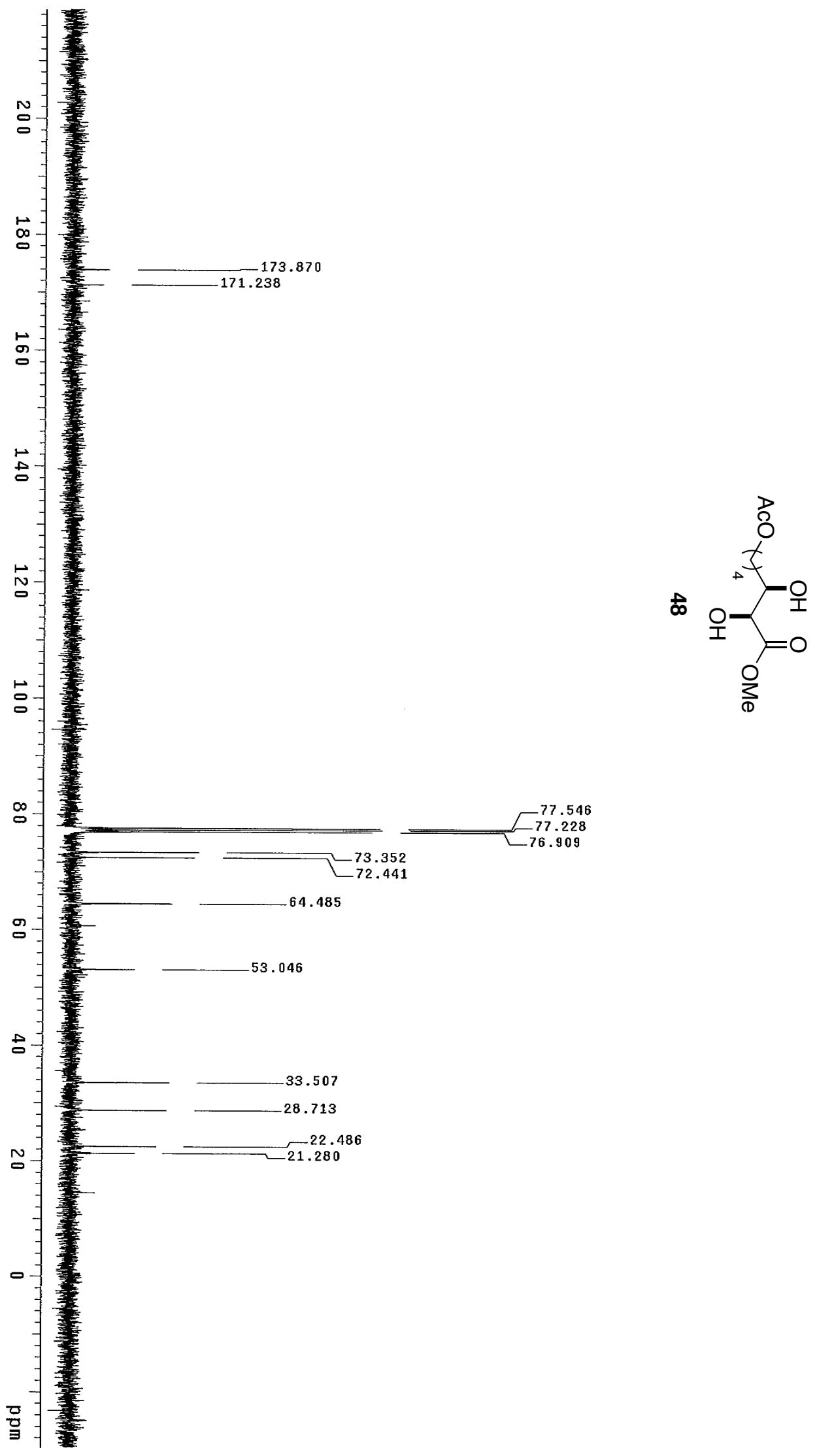




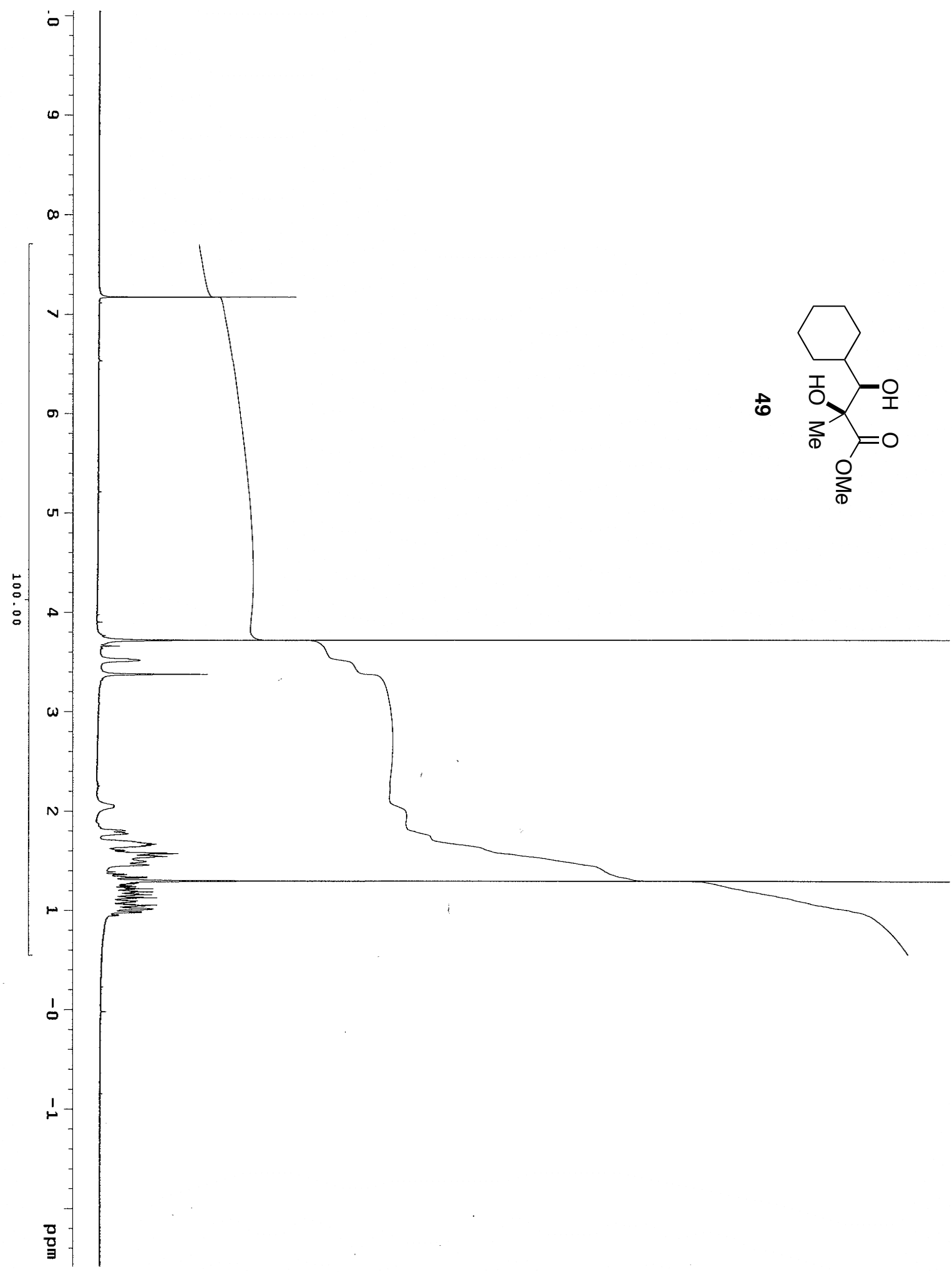



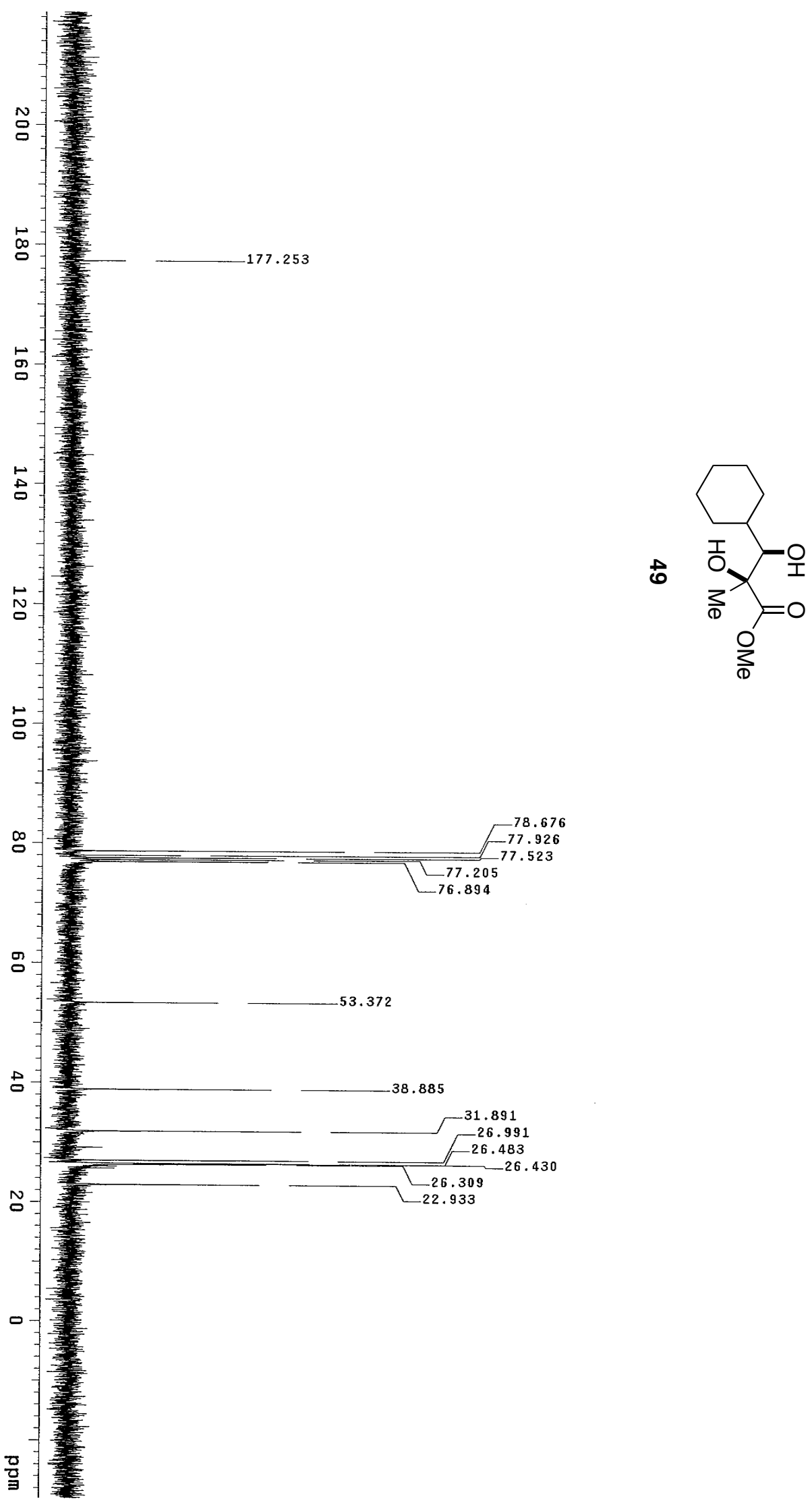


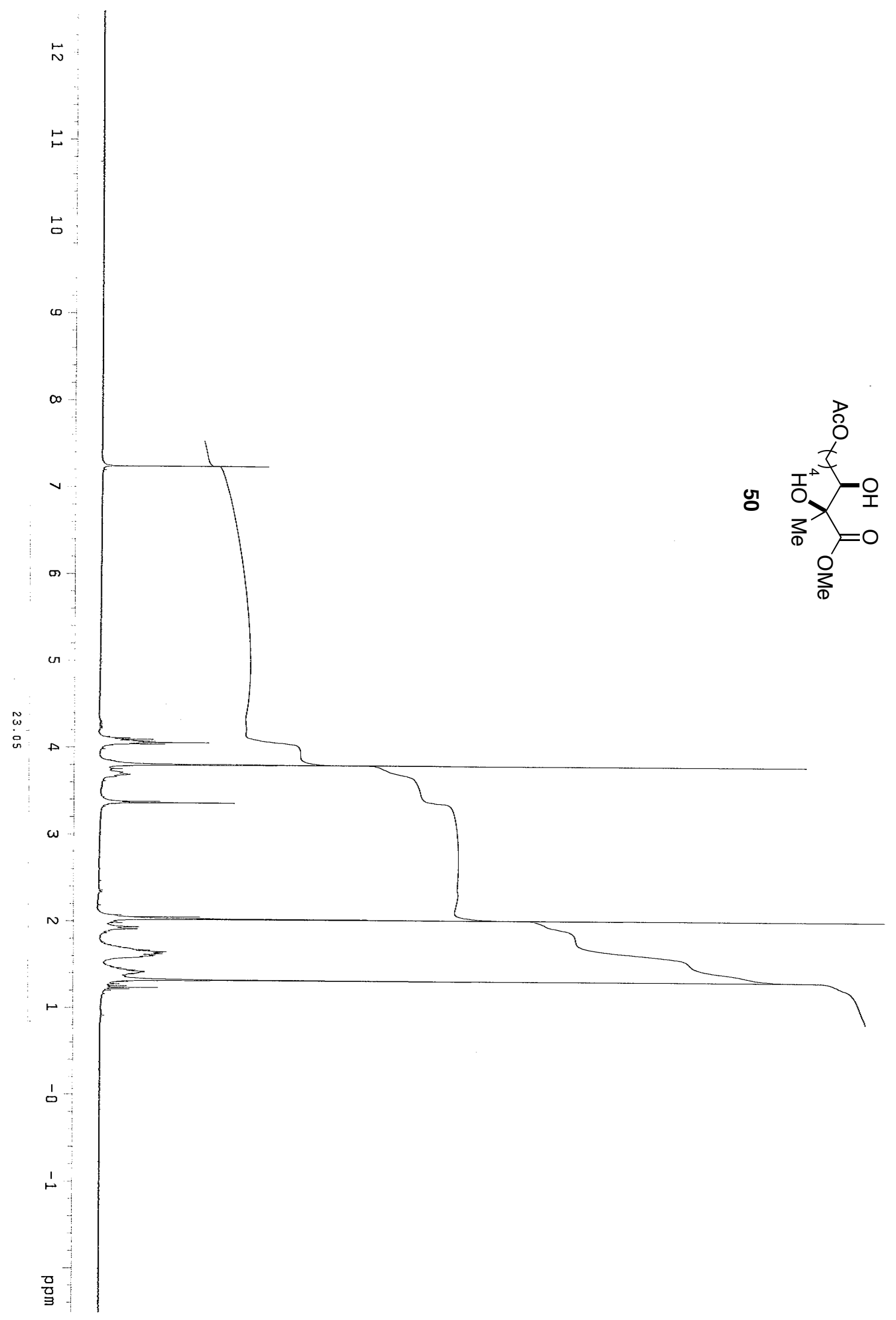




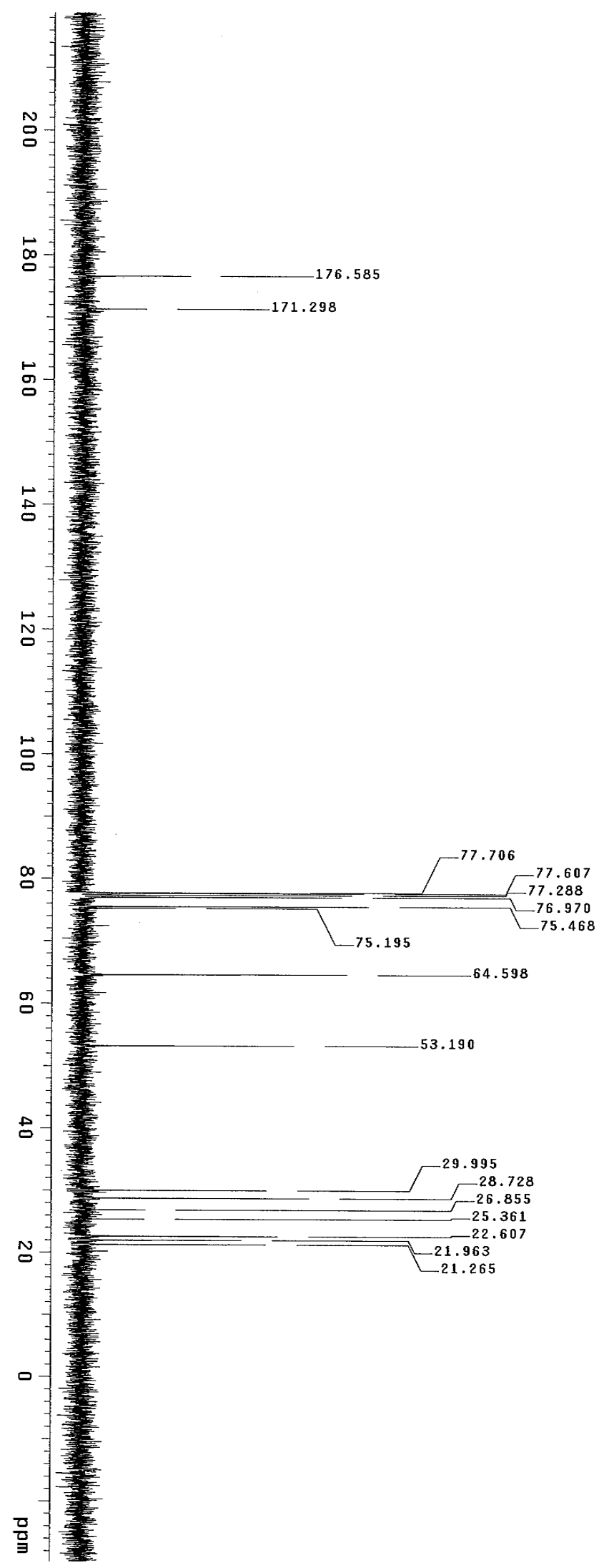

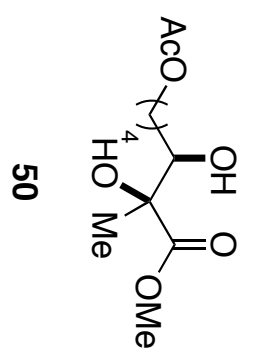




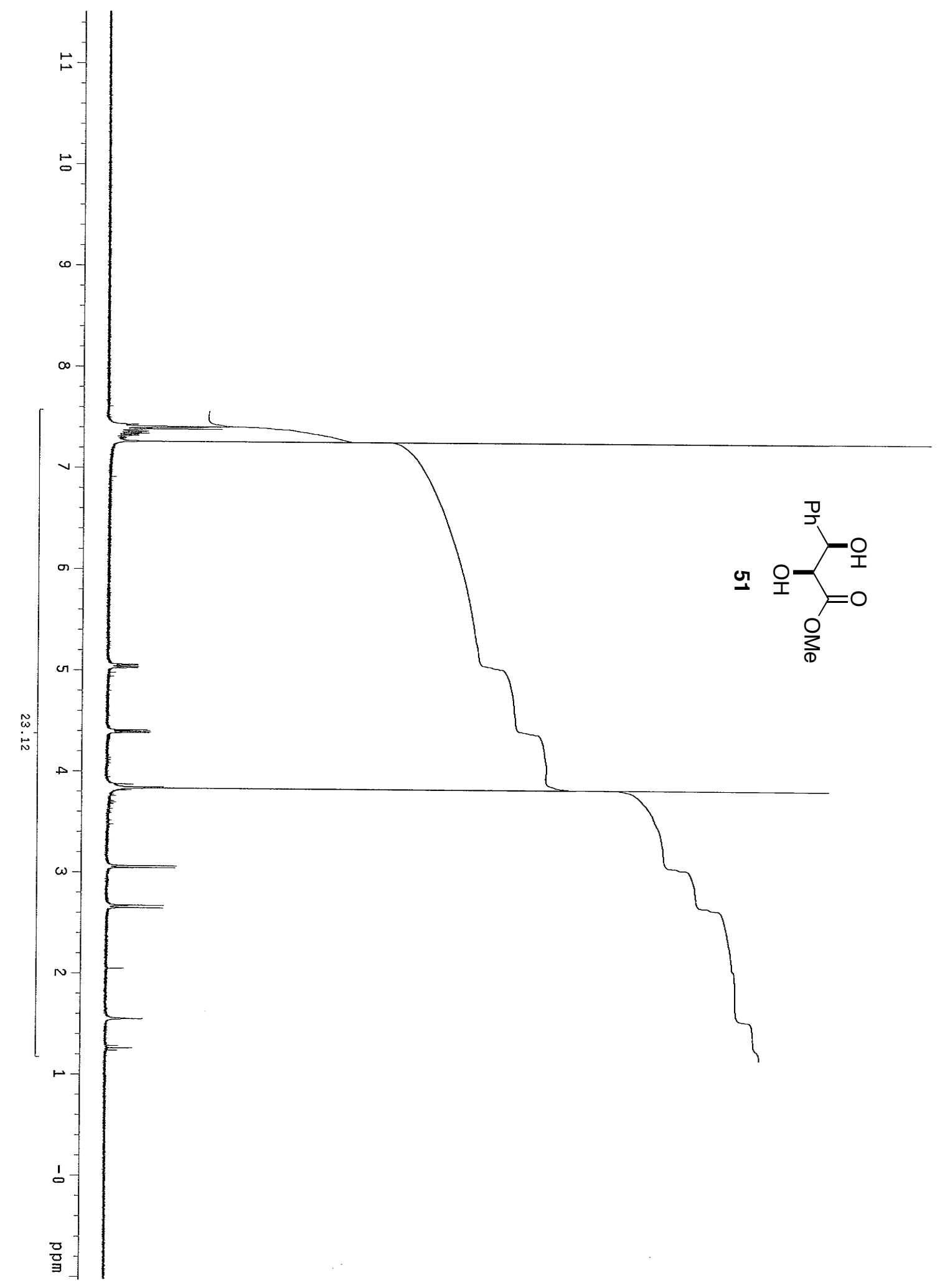




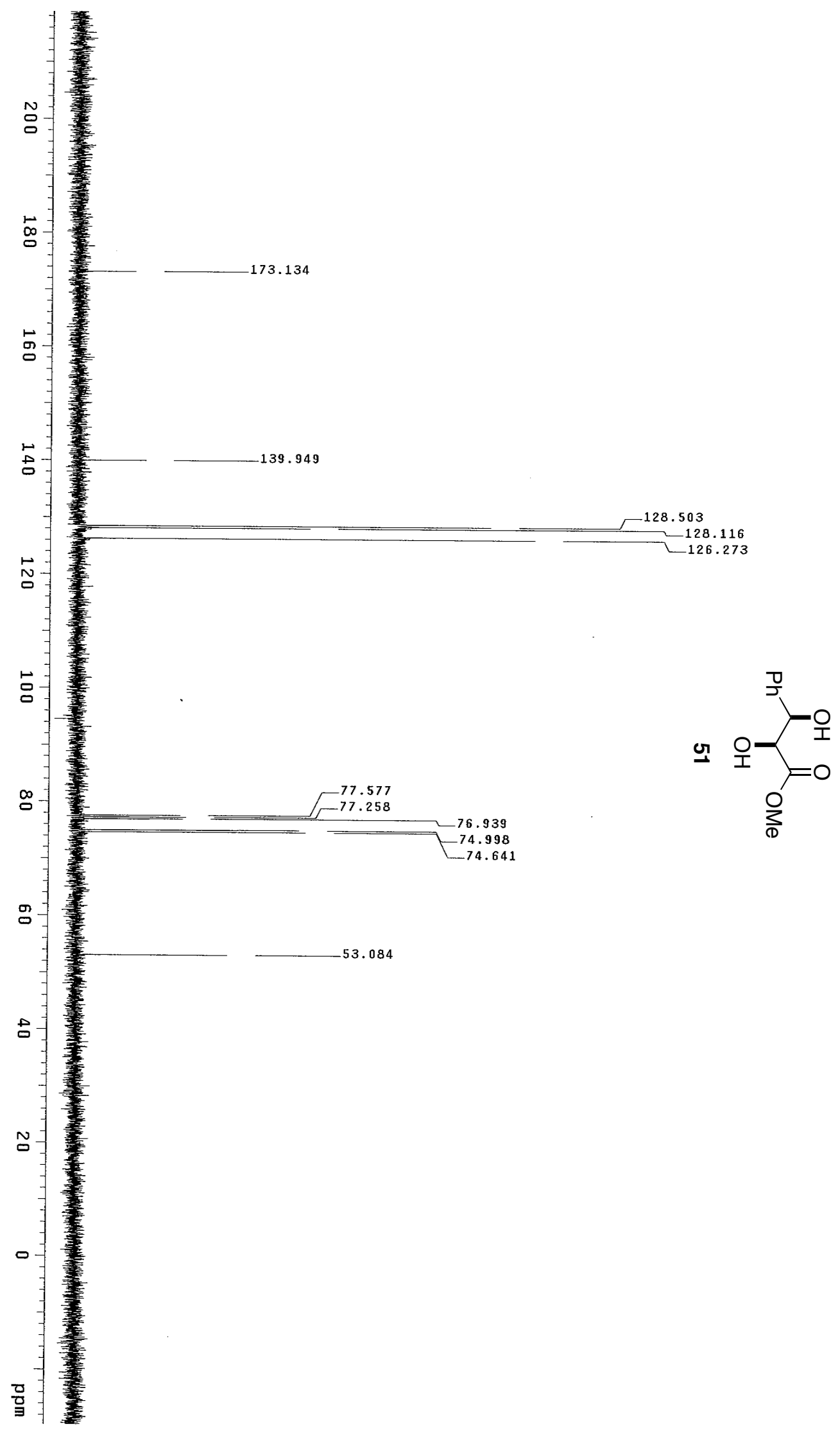




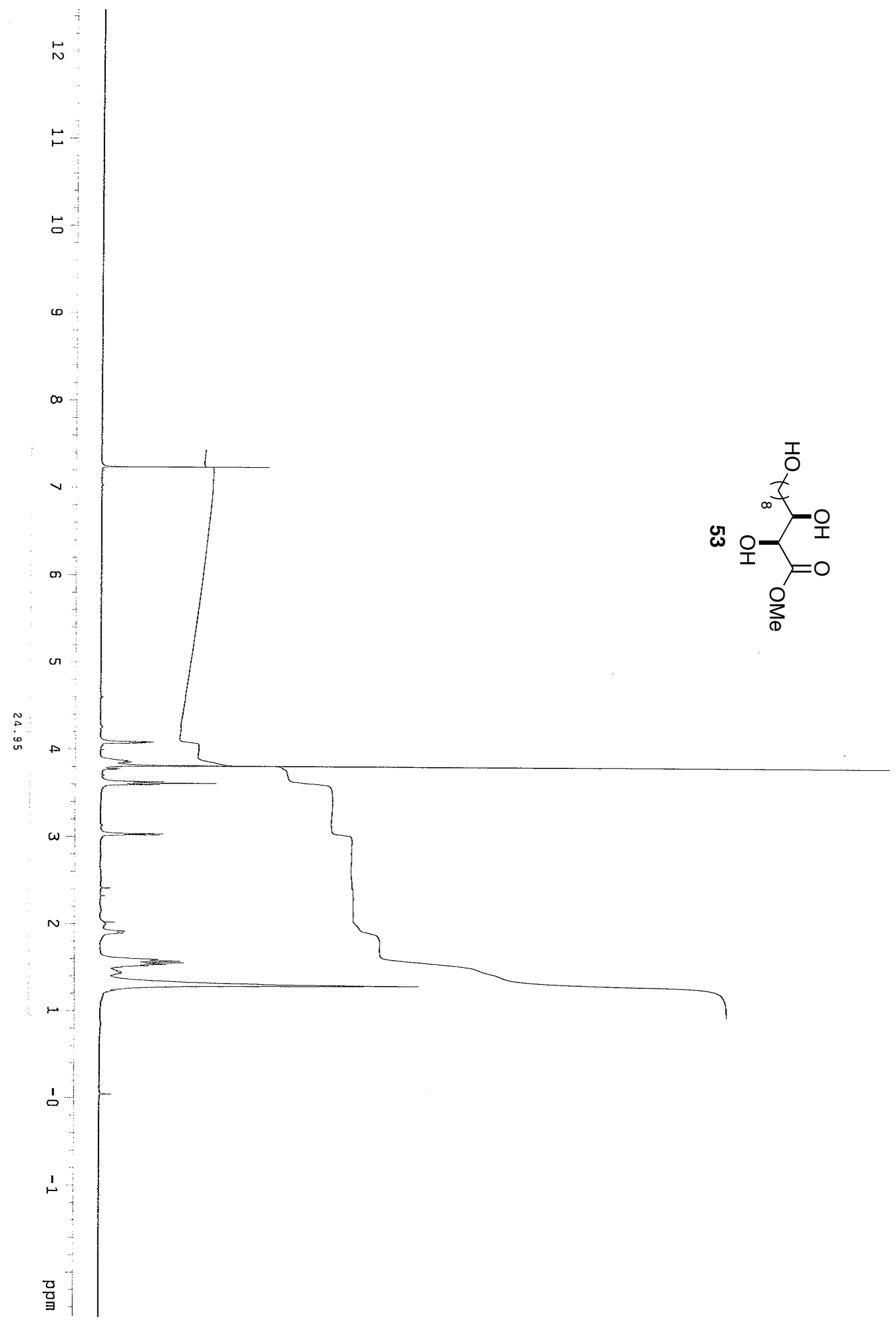




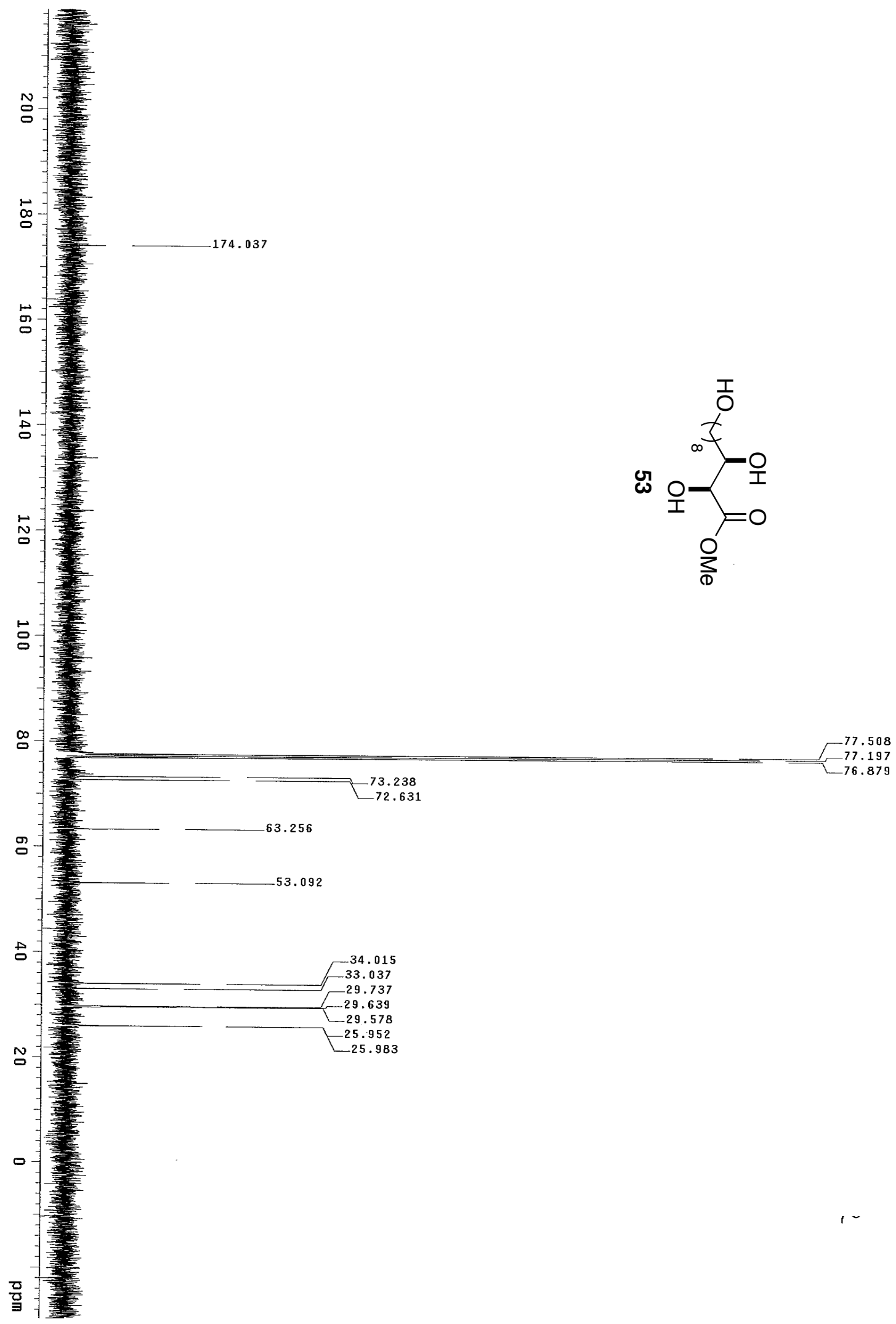




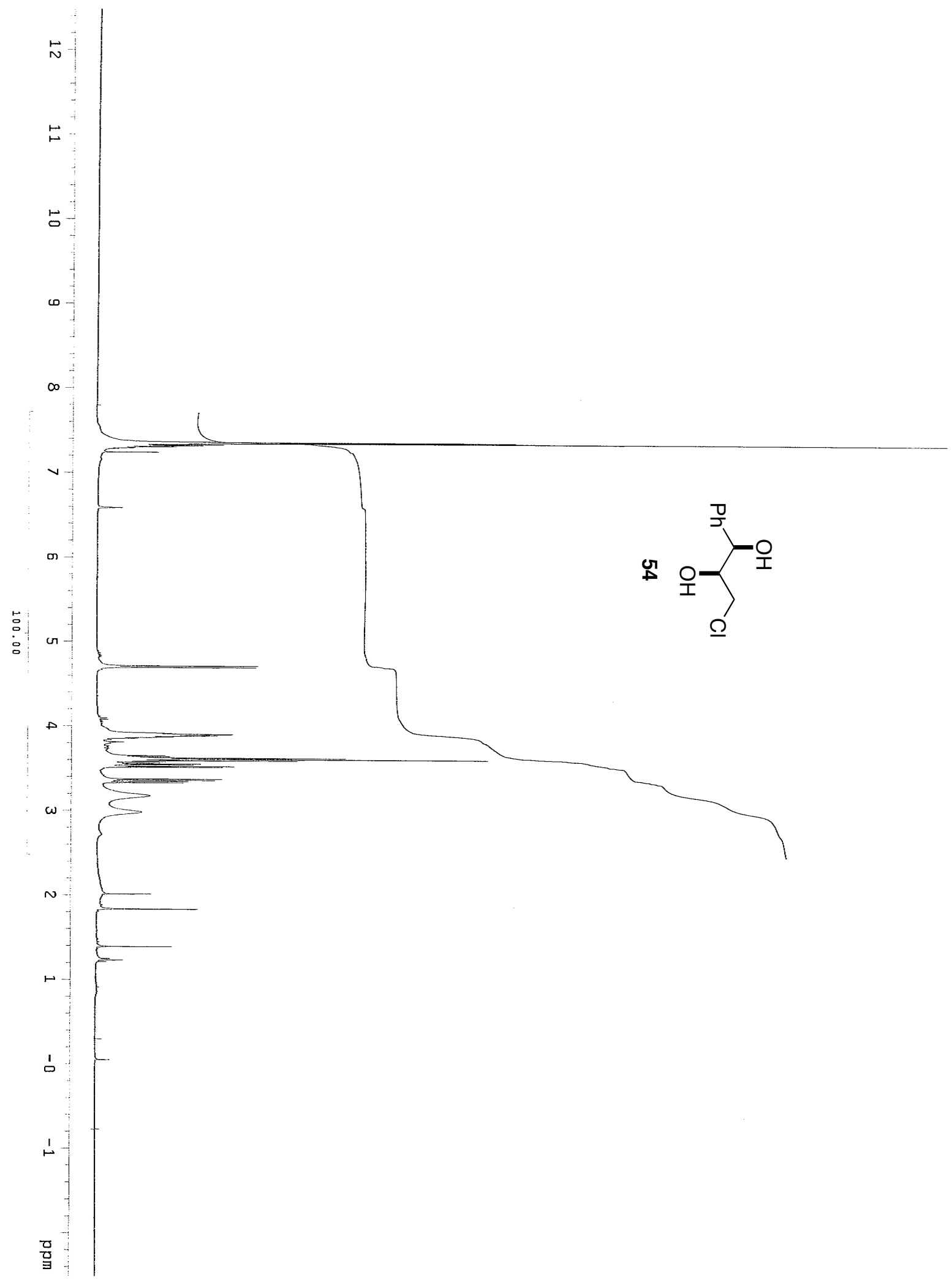




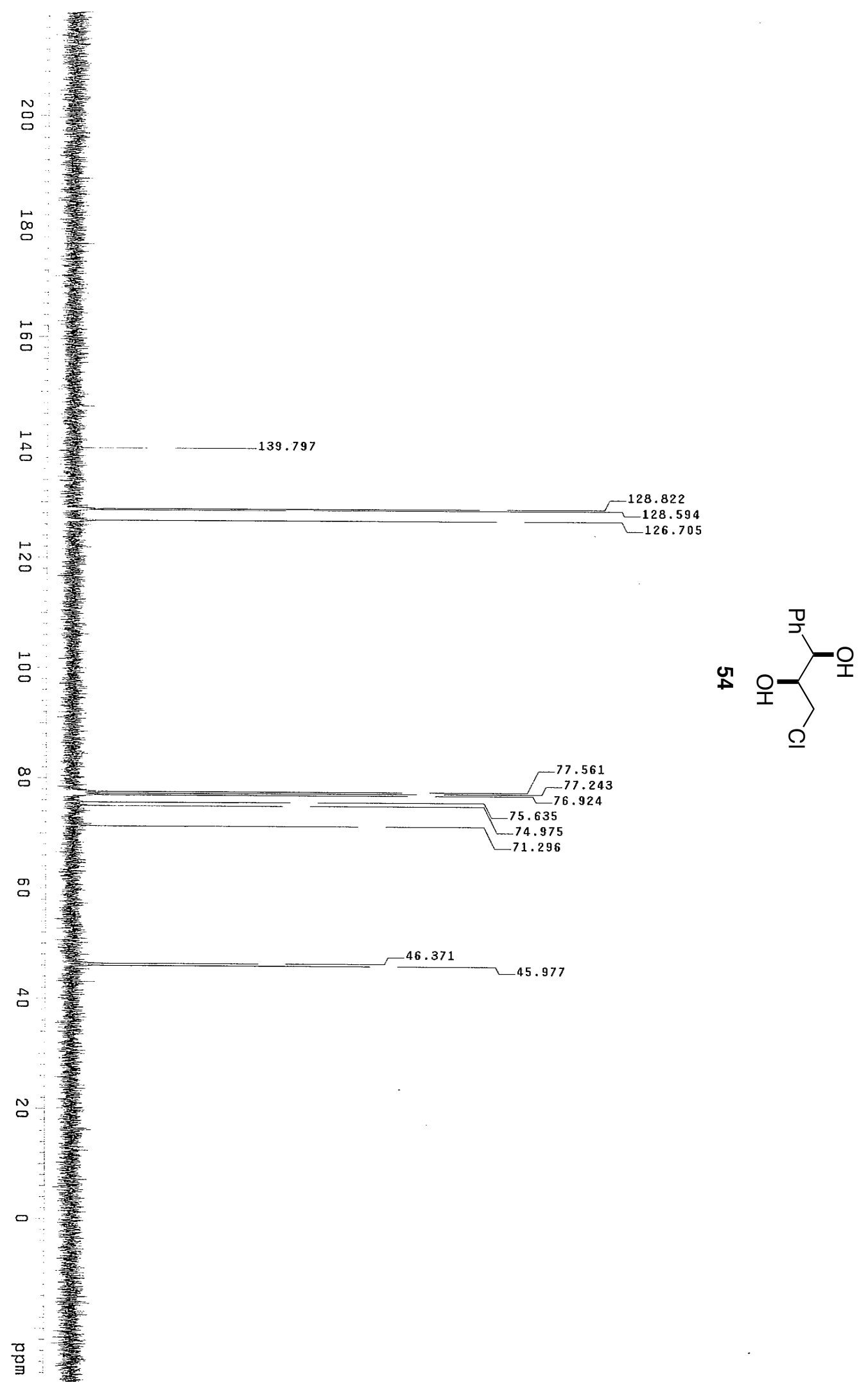




\section{References}

1. Plietker, B. Eur. J. Org. Chem. 2005, 1919.

2. Plietker, B. J. Org. Chem. 2003, 68, 7123.

3. Plietker, B. J. Org. Chem. 2004, 69, 8287.

4. Plietker, B. Org. Lett. 2004, 6, 289.

5. Beligny, S.; Eibauer, S.; Maechling, S.; Blechert, S. Angew. Chem. Int. Ed. 2006, 45, 1900.

6. Plietker, B.; Niggemann, M. Org. Lett. 2003, 5, 3353.

7. Martin, V. S.; Ode, J. M.; Palazon, J. M.; Soler, M. A. Tetrahedron-Asym. 1992, 3, 573.

8. Plietker, B.; Niggemann, M.; Pollrich, A. Org. Biomol. Chem. 2004, 2, 1116. 